\title{
Les réalisations \\ d'Electricité de France concernant l'énergie marémotrice
}

PAR

René Bonnefille

Direction des Etudes et Recherches Electricité de France

\section{Sommaire}

\section{AVANT-PROPOS.}

\section{L'ÉNERGIE MAREMOTRICE.}

1.1. Principe de fonctionnement des usines marémotrices.

1.2. L'énergie naturelle d'un site.

1.3. Les usines marémotrices dans le monde.

1.3.1. Projet américain en Alaska.

1.3.2. Projet U.S.A.-Canada de Passamaquoddy.

1.3.3. Projet canadien de la baie de Fundy.

1.3.4. Projets soviétiques.

1.3.5. Projet anglais de la Severn.

1.3.6. Projets français.

\section{LA RANCE.}

2.1. Historique.

2.2. Le site.

2.3. Réalisation de l'usine.

2.3.1. Les études théoriques du S.E.U.M.

2.3.2. Les modèles réduits.

2.3.3. Les travaux en bureau d'études.

2.4. Les groupes bulbes.

2.4.1. Historique.

2.4.2. Le groupe expérimental de Saint-Malo.

2.4.3. Les groupes de la Rance.

2.4.4. L'évolution des groupes bulbes.

2.5. Les études de corrosion

2.5.1. Etudes des peintures.

2.5.2. Les alliages.

2.5.3. La protection cathodique.
2.6. L'exploitation de l'usine de la Rance.

2.6.1. Théorie des cycles d'exploitation.

2.6.2. Le principe de calcul des éléments de la méthode de la Programmation Dynamique.

2.6.3. Les limitations.

2.6.4. Recherches du fonctionnement optimal.

2.6.5. Prise en compte des aléas.

2.7. Les performances de la Rance.

2.8. Analyse du fonctionnement de l'année 1973.

2.9. Conclusions sur la Rance.

\section{LE PROJET DES ÍleS CHAUSEY.}

3.1. Les études océanographiques.

3.1.1. La marée.

3.1.2. La houle.

3.2. Les études hydrauliques.

3.2.1. Les difficultés.

3.2.2. Les modèles réduits.

3.2.3. Les principaux résultats.

3.3. Les études géologiques.

3.3.1. Mesures bathymétriques.

3.3.2. Mesures géophysiques.

3.3.3. Mesures géologiques.

3.3.4. Interprétation géologique.

3.3.5. Recherche des carrieres.

3.3.6. Etude sédimentologique.

3.3.7. Conclusion.

3.4. L'historique des avant-projets de l'usine de Chausey.

3.4.1. Les projets antérieurs à 1950.

3.4.2. Les projets de 1950 à 1955.

3.4.3. Réflexions sur les projets antérieurs à 1955.

3.4.4. Les projets postérieurs à 1955.

3.4.5. Réflexions sur les projets postérieurs à 1955.

4. LES MINQUIERS.

BIBLIOGRAPHIE.

PLANCHES. 


\section{Avant-propos}

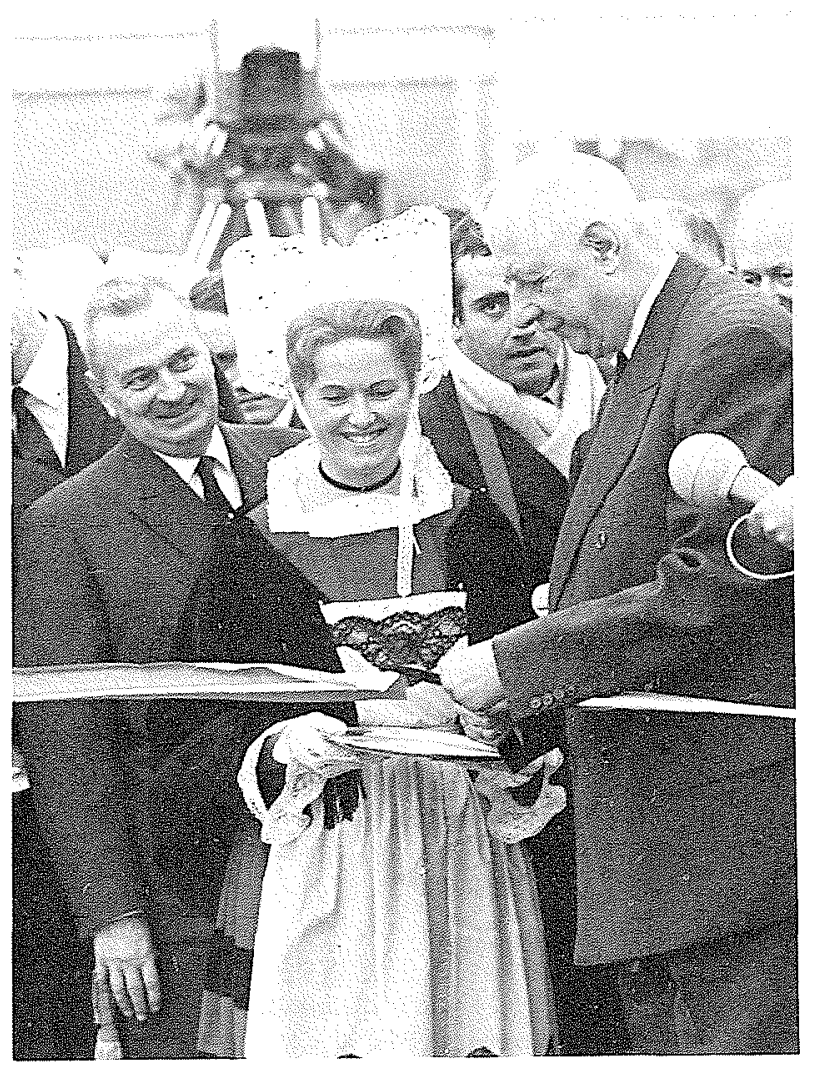

Le 26 novembre 1966, le général de Gaulle, Président de la République Française, inaugurait l'usine de la Rance; il marquait ainsi le début de la réalisation d'un vieux rêve des hommes, la domination de l'énergie des marées, rêve ébauché par les moulins à marée et déjà esquissé dans le Traité d'Architecture Hydraulique de Bélidor [1].

L'inauguration de l'usine couronnait de succès le résultat d'une vingtaine d'années de patience et de travail d'une équipe animée par Robert Gibrat. La France se trouvait dotée de la première usine marémotrice du monde.

Aujourd'hui, huit ans après sa mise en service, l'usine, avec ses $0,24 \mathrm{GW}$ installé, sa production annuelle de $500 \mathrm{GWh}$, est bien peu de chose dans le parc de production d'Electricité de France. Pourtant, elle suscite toujours beaucoup d'intérêt; elle redevient "vedette » en tant que témoignage de la maîtrise d'une forme d'énergie dont on s'accorde pour dire qu'elle est gratuite et peu polluante.

Comme la France est un des pays les plus riches en énergie marémotrice, il est normal d'essayer d'éclairer l'opinion sur les réalisations d'Electricité de France dans ce domaine; en particulier où en sommes-nous des projets ambitieux d'une usine marémotrice dans la baie, du Mont-Saint-Michel?

Le nombre d'ouvrages, rapports, articles, communications relatifs à l'énergie marémotrice en France, dépasse la centaine, la plupart publiés entre 1950 et 1966.
Nous renonçons à les citer tous; la bibliographie in fine ne mentionne que quelques articles. L'attention du lecteur est cependant attirée sur les ouvrages de synthèses:

- les thèses de M. Philipponneau [9], L. Vantroys [18] et R. Bonnefille [38];

- l'ouvrage de R. Gibrat, l'Energie des marées [32];

- les comptes rendus des Quatrièmes Journées de l'Hydraulique [11], les numéros spéciaux de La Houille Blanche $[27,44]$ et de la Revue Française de l'Energie [33];

- le rapport final des études sur modèle réduit de la Rance [37].

\section{L'énergie marémotrice}

Soumises aux attractions alternatives du Soleil et de la Lune, les masses d'eau océaniques oscillent en résonance avec des amplitudes relativement faibles. Ces ondes-marées se propagent dans les océans jusqu'aux mers littorales à faibles profondeurs où leur amplitude grandit.

Une onde-marée est le siège d'échanges d'énergies cinétique et potentielle; l'utilisation de l'énergie cinétique des courants de marée est difficile; en revanche l'énergie potentielle correspondant à la surélévation du niveau de l'eau peut être transformée aisément en énergie mécanique grâce au couple barrage-turbine.

Cette énergie est cependant limitée; la puissance dissipée par les marées du Globe terrestre est de l'ordre de 3 TW [24], dont un tiers est dissipé dans les mers littorales; en admettant qu'on sache en utiliser $20 \%$, l'énergie annuelle d'origine marémotrice que l'on obtiendrait serait de l'ordre de $400 \mathrm{TWh}$. Il ne faut donc pas compter sur l'énergie marémotrice pour satisfaire les besoins énergétiques mondiaux; cependant, en certains sites privilégiés, où de grandes puissances peuvent être installées, cette forme d'énergie, entraînant peu de troubles écologiques, mérite d'être considérée. A cet effet, la figure 1 donne un aperçu des zones où les marées ont une grande amplitude et où des sites marémoteurs sont envisageables.

\subsection{Principe de fonctionnement des usines marémotrices}

Le principe de base de l'utilisation de l'énergie marémotrice est de soustraire à la mer, au moyen de barrages, une ou plusieurs zones marines, transformées en réservoir, de façon à obtenir une différence de niveau de part et d'autre d'un barrage-usine. Comme le niveau de la mer varie, avec une période de l'ordre de grandeur de la demi-journée sur nos côtes, à moins de prendre des précautions particulières la chute disponible varie de même, et surtout s'annule périodiquement.

La figure 2 illustre le schéma de fonctionnement d'une usine marémotrice édifiée à l'entrée d'un estuaire ou d'une rade et utilisant la chute entre le niveau de la 
mer descendante et le niveau du bassin rempli à marée montante. En admettant, en première approximation, le débit de turbinage constant pendant la phase $\mathrm{CA}^{\prime}$, l'énergie produite, proportionnelle au produit du débit par la chute et par le temps, est représentée par la surface hachurée. Ce type de cycle, dit "à simple effet », a pour lui la simplicité ; mais l'énergie est produite de façon très discontinue, quoique d'une manière certaine, puisqu'il est toujours possible de prévoir les heures de production. Le cycle "à double effet " résulte de la superposition de deux cycles à simple effet au vidage et au remplissage du bassin (fig. 3). La production est moins discontinue; mais il subsiste des périodes improductives quand les niveaux de la mer et du bassin sont à des cotes voisines. Dans ce type de fonctionnement, il est nécessaire que les turbines et les vannes puissent fonctionner dans les deux sens de l'écoulement.

La possibilité de faire fonctionner les turbines en pompes, à des époques judicieuses de la marée, permet de tirer le meilleur parti de l'énergie marémotrice, dans un but soit de souplesse, soit de profit économique. Il suffit de substituer aux époques d'attente une phase de pompage, dans la mesure où l'énergie est alors bon marché, pour :

- soit surélever le niveau du bassin à pleine mer;

- soit abaisser le niveau du bassin à basse mer, ce qui est difficile pour des raisons tant hydrauliques qu'écologiques aux niveaux très bas.

La figure 4 montre l'intérêt économique du projet par comparaison des énergies de pompage (aires $\mathrm{AGG}^{\prime}$ ou $\mathrm{DHH}^{\prime}$ ) et des accroissements d'énergie produite (aires $\mathrm{B}^{\prime} \mathrm{C}^{\prime} \mathrm{C}^{\prime \prime} \mathrm{B}^{\prime \prime}$ ou GFF"E").

Ces considérations, ajoutées aux variations des amplitudes de la marée, montrent I" "infinie " variété de cycles de fonctionnement ( $3.10^{32}$ entre deux vives-eaux). Le choix optimal de la suite de ces divers cycles fut jadis considéré comme un problème ardu $[4,8,27 \mathrm{~h}]$. Nous verrons comment il a été effectivement réalisé à la Rance $[44 a]$.

Les usines à un seul bassin ont pour elles la souplesse; moyennant des périodes d'attente et de pompage convenables, elles fonctionnent très bien en usine de pointe. En revanche, leur puissance garantie est "faible ». Pour remédier à cet inconvénient, qui ne se présente que dans le cas d'une usine alimentant un réseau isolé, il suffit de disposer de deux bassins entre lesquels est maintenue une différence de niveau, l'un étant rempli à pleine mer et l'autre vidé à basse mer (fig. 5). Dans ce cas, une production minimale d'énergie est garantie; le pompage a pour effet d'augmenter la part de celle-ci. Mais, d'une part les ouvrages sont plus conséquents que dans le cas d'un seul bassin, d'autre part les pompes indépendantes des turbines sont très peu utilisées; enfin les niveaux dans les bassins ne retrouvent plus leur gamme naturelle de variation.

\subsection{L'énergie naturelle d'un site}

Quelle est l'énergie marémotrice que l'on peut espérer tirer d'un site donné? Cette question est à la base de toutes considérations sur l'utilisation de l'énergie des marées. Elle n'a pas encore reçu de réponse satisfaisante; R. Gibart [8] a dégagé la notion d'énergie natu- relle, notion qui malheureusement n'est qu'un indice et non pas un critère. Par exemple, la formule de Betz donne la limite supérieure de l'énergie éolienne théoriquement utilisable sur un site; dans ce cas une théorie a pu être bâtie, car il s'agit d'un écoulement fluide permanent. Il n'en est pas de même en matière d'énergie marémotrice, car les sites intéressants, à grands marnages, sont des zones de résonance des ondes-marées; l'usine peut accentuer ou détruire la résonance; le pompage, utilisant une énergie extérieure, remet tout le bilan énergétique en question.

Un raisonnement «simpliste» pour définir l'énergie naturelle consiste à considérer les tranches de fluide du bassin à la cote $z$ au-dessus du niveau des plus basses mers, où la surface du bassin est $S(z)$. Chaque tranche peut produire en se vidant une énergie

$$
d E=\rho g z S(z) d z
$$

$\rho$ et $g$ désignant respectivement la masse volumique du fluide et l'accélération de la pesanteur. Dans la mesure où tout le bassin pourrait se vider instantanément, à l'étale de basse mer, ce qui est un maximum techniquement et hydrauliquement impossible, l'énergie totale de vidage serait :

$$
E_{v}=\rho g \int_{0}^{1} S(z) z d z
$$

A désignant le marnage maximal, différence entre la cote de basse mer et la cote de pleine mer à laquelle le bassin a été rempli. Le remplissage, supposé encore instantané, pourrait produire aussi de l'énergie:

$$
E_{R}=\rho g \int_{0}^{1} S(z)(A-z) d z
$$

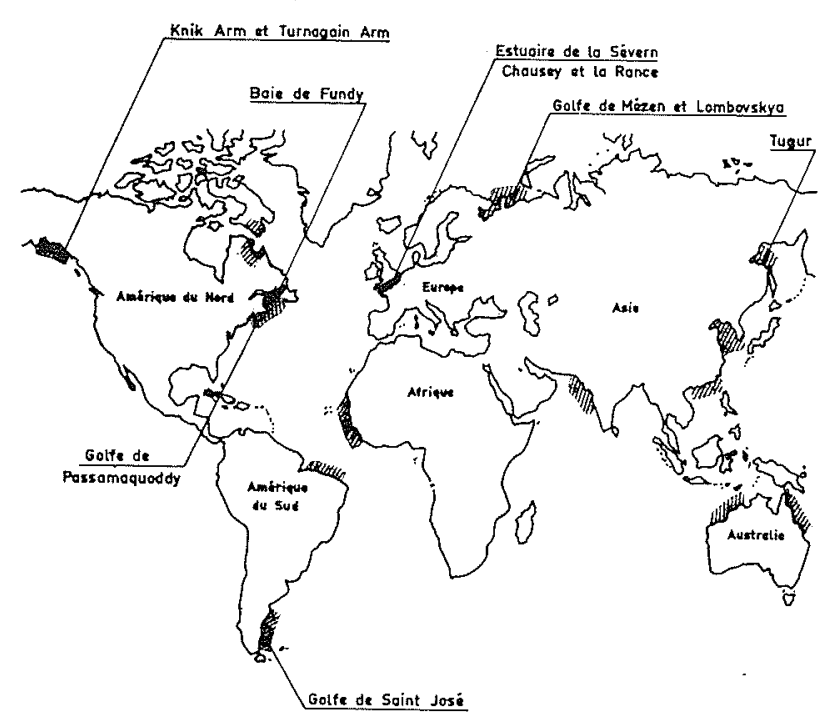

ZONES DE MAREE MOYENNE SUPERIEURE A $4 \mathrm{~m}$ "yIIIIF

1/ Emplacement des grands sltes marémoteurs 
côté d'Aval prife depuis le radier jufqu’à la hauteur du rez-de chauffée, où l'on diftingue les differentes parties quel'on peut voir dans l'enfoncement depuis l'entrée de l'eau jufqu'à fa fortic. Pour les reconnoítre il ne faut que chercher les chiffres \& les lettres femblables répandues dans les differentes figures, qui font voir la relarion qu'elles ont entrelles puifqu'elles répondent aux mêmeschofes vûes de differens fens; la troifíme figure montre le plan du radier du côté d'Aval, \& de quelle maniere font affémblées les pieces de charpente fur lefquelles on a établi la maçonnerie qui compofe les tomeaux; car comme l'cau paffe par deffous, il a fallu les porter en l'air \& fe contenter d'appuyer leur bafe fur les piles Y.

La deuxiéme figure fait voir le plan du rez-de-chauffée du moulin où font placez les meules, \& l'affemblage des pieces de charpente qui en font la féparation: elles font difpofées de maniere qu'on peut démontcr tout ce qui apparrient à une des meules quand il y a quelque réfararion à faire fans interrompre le travail des au. tres, ayant chacune leur courfier qu'il fuffit de fermer pour avoir la liberé de manouvrer haut \& bas.

Comme il n'y a que 5 pieds 4 pouces du centre d'une meulè̀ celui de l'autre fur une riviere de ro à i i toifes de large, on peut en placer jufquà i 2 , au lieu qu'ordinairement on n'en met que 4 encore faut-il faire deux bîtincons, un fur chaque bord; icy il n'y a ni rouct ni lanterne, \& par confequent d'autre frottement que celui du pivot de la roue, ce qui rend les réparations moins fréquentes : cetre rove qui n'a que 3 picds de diamétre eft comporée d'une feule picce ; pour la faire, on prend un tronçon d'un gros arbre, \& on y taille les aubes que l'on incline fur fon ćpaifeur, jes faifant un peu courbes comme on le peut voir par la quatrićme \& cinquiéne figure qu'on a rapporté en grand pour la rendre plus fenfible.

Pour domer à cette roue toure la perfection dont elle me parcí fufceptible, il y auroit plufieurs recherches curieufes à faire alifquelles je ne na areterai point : je dirai feulement que l'cau quila pouffe la fait agir arce une force compofée de l'action de ta pefanteur \& de la direction circulaire que le rommeau lui donne; quo la courbure des aubes devroir fuivre celle de la développée e un cercle, \& que l'oblicuité qu'clles ont de haut en bas devroit faire aveclarbre qui leur fert d'efficu un angle de 55 dégrez, puifque ces aubes font dans le mêne cas que les ailes d'un moulin à vent.

670 . Il me refte à décrire une autre efpece de Moulin dont io fojervir du crois quaucun Auteur n’a parlé, étant nouvelle \& peu connue:

\$06 Architecture Hydraulique, Livre II

HFI \& va s'écouler à la mer ; ainfi toute la manœuvre fe réduit à ouvrir \& a fermer alternativenent tous les fix heures les vamnes $\mathrm{E}$, $B, \&$ D , E. Pour interrompre le moulin quand on le juge à propos, l'oun a placé une vamne à l'endroit A qui empêche que la me ne paffe aude-là.

La feconde figure comprend deux moulins qui agiffent de la même maniere que le précedent, mais avec un peu plus de circuir ; l'on fuppoie que le cóté $L$ répond au rivage \& le côté $K$ au refervoir: Quand la mer nonte, l'on ouvre les trois vames $A, G, C$ Q l'on ferme les rrois autres $B, D, H$; ainfi l'eau fait tourner d'abord la rout $F$, enfuite l'autre $E$, de-lì paffe dans le canal $\mathrm{MCI}$ pour ferendre au refervoir.

Quand la mer baiffe on ferme les trois pafages $A, G, C, \&$ l'on ouvre les trois autres BDH, l'eau du refervoir vient faire toumer la roue E de même fens quauparavant, de-là coule par le paffago $D$, \& va faire tourner la toue $F$ comme en premier lieu, enfuit elle s'échappe par le canal PBO, \& va fe jetrer à la mer; ainfi la manouuve confifte à ouvrir \& à fermer alternativement les vannes.

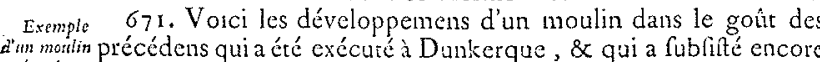
cxicuté ari- long-tems après la démolition; n'ayantété déruit que depuis quelDrifriserque ques années parle proprietaire même, picqué de voir qu'on vou-

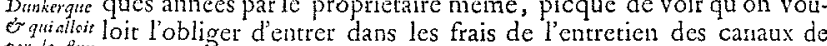

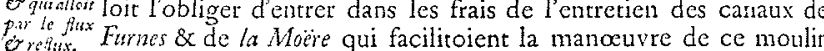
fitué dans la Ville entre ces deux canaux. Il faur être prévenu quo le fond du canal de la Moère eft de niveau avec l'ancien port, \& que le fond de celui de Furnes eft de 6 pieds plus élevé; ainfi le moulin manocuvroit à la marée montante par le canal de la Moëre, \& continuoit à la marée defcendante par celui de Furnes de la maniere du monde la plus commode comme on en va juger.

Pean. 8. Ce moulin contenoit huit meules marquées $\mathrm{H}$, dont 6 tournoient par le moyen de la mer, \& les deux autres par celui du vent ; c'eft pourquoi on a pratiqué la gallerie de charpente KL en dehors de la Tour pour difpofer l'axe des áles dans la direction duvent.

Le plan fait voir trois courfiers $A, B, C$, dans chacun defquels toumoit une roue qui donnoit le mouvement à deux meules, comme le profil le fait affez fentir. Je ne dis rien du mouvement de la roue $\mathrm{F}$, qui répondant dans le courfier $\mathrm{C}$, avoit la liberté de tourner tantôt d'un fens, tantôt d'un autre fuivant le flux \& le reflux pour ne miarrêter qu'aux deux autres répondans aux courfiers $A$
Chap. I. Des Mouzins a Eac. 305 eile fe réduit à faire enforte d'affujettir le fux \& le reflux de la mer furx as a pour faire tourner des roues toujours du mêtme fens, ce qui s'exé- mir , furt. cure d'une maniere fort ingenieufe; l'on en atrribue la premiere ${ }_{x i r}$ drs invention à un nommé Perfe Mâtre Charpentier de Dunkerque, rems fousqui mérite affurément beaucoup d'ćloge, n'y ayant point de gloirc niors falis. plus digne d'un bon Citoyen que de produive quelqu'inencition wile à la focieté. En effet, combien n’y a-tili poin de choles eflenrielles à la vie, dont on ne comnoit le prix que quand on ell eft privé; les moulins en general font dans ce cas-là, l'on doit fçavoir bon gréa ccux qui nous on mis en ćrat d'en confruire par tout : par exemple à Calais, comme il n'y ferpente point de rivieres, on n'y a point fait jufqu'ici de moulins à cau, \& ccux qui vont par le vent chomant une partic de l'année, il y a des tems oì cette Ville fe trouve fans farme, \& j'ai vâ la Garnifon en 1730 obligée de faire venir du pain de Saine Oner, au licu qu'en fe fervant du fux \& reflux de la ner, on pourroit conftruire autant de movilizs a cau que l'on roudroit ; il y a d'autres Villes dans le voifinage de la mer fujettes au même inconvenient, parce qu'apparemment elies ignorent le moyen d'y remédie:. C'eft principalement en lcur faycur que jay écrit ce qui fuit.

La figure premiere comprend trois canaux dont celui du milicu prast. $\%$ KCM fe ferme avec deux vannes placées aux endroits $B$ \& $E$, les $F$ deux autres GDL \& HFI fe ferment aufi parles vannes $D \& F$, pour entendre la manceuvre qui fait aller le moulin dont la rouc eft placée en C, on fuppofe que l'eau de la mer entre du côté de $M$, \& forr du cóté de $\mathrm{K}$ pour s'aller rendre dans un grand refervoir oì elle refte en dépót.

Quand la mer monte, on leve les vannes B, E, \& l'on baiffe les deux autres $D$ \& $F$; alors l'eau paffant par le canal du milié fair tourner la roue cruiron 4 heures \& - des 6 que la mer employe àmonter, parce que lorfqu'elle approche de fe mettre de niveas avec l'cau du refervoir, Ia roue cefie de tourner pendant we heure \& demie arant que la maxée ait arcein fa plus grande haurcar, \& encore une heure \& demie aprés: ainfi des 12 henres que comprond le :ems du fiux \& reflux, il y en a 3 pendant lerquelles lo moulin chome.

Quand la mer commence à baiffer, l'on ferme les vames E \& B, \& fon ourre les deux aurres D \& F ; l'eau du refervoir en contrainre de paffer dans le canal GDL, \& ne pouvant s'echapper do cóté de la mer, elle vicne paffer fous la roue $\mathrm{C}$ qu'cilic fait tourner du même fens qu'auparavant: de là clle s'échappe par le canal Qqiij
CHAP. I DES MOULINSA EAV. 307 \& B dont l'équipage de chacune eft repréfentée par le fecond profil. Pour leur donner le mouvement, on a fait quatre porres à deux battans $D, E, F, G$, qui s'ouvroient \& fe fermoient alternativement d'elles-mênes par l'action de l'eau. Par exemple, à la marée montante les portes $E$ \& $F$ s'ouvroient, \& les deux autecs $D \& G$ fe fermoient, l'eau venant pafrer par les courtiers du fens maroue partes fléches faifoir tourner les roues pendant le flux; \& à la marée defcendante les portes $G \& D$ s'ouvroient, \& les deux premieres $E$ \& $F$ fe fermolent ; l'eau fe trouvant arrêtée en $E$ pafioit PraN. $\rightarrow$. par la porte $G, \&$ fortoit par l'entrée $D$, après avoir fait toumer les FIG. $\$$ deux roucs du même fens qu'auparavant.

672. J'ai fait réflexion que l'on pouvoit fe fervir de la marée pour faire aller des moulins d'une maniere encore plus fimple cue ceile surere max que je viens de décrire. Je fuppofe que RST marque la bafle mer, forvir dit

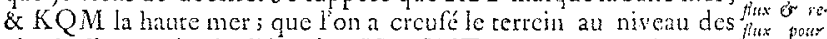
plus bafies marćes fur l'écendue SLA GNT, qui aboutit à deux re-faire poust fervorrs DOH \& GPI, dont le lit du premier doir êre de 6 ou $7^{\text {ner }}$ des pieds plus ćlevé que celui du fécond qu'on fera de niveau avec la baffe mer; ainfi l'on ménagera une chute à l'endroit $\mathrm{HI}$, accompagnée d'une éclufe fermée avec des vannes pour foutenir les eaux du canal fuperieur \& faire tourner plufieurs moulins : à l'entrée du bafin fuperieur il faudra faire une éclufe $A B$ fermée par deux portes bufquées $\mathrm{D}$ qui s'ouvriront d'elles-mêmes du córé du canal à la marée montante; l'eau entrera à la hauteur de 7 à 8 pieds, \& s'y trouvera enfermzé fans en pouvoir fortir quen ouvrant les pertuis des moulins, parce que les portes D fe refermerone d'elles-mêmes auffi-tót que la mer commencera à baiffer.

On conftruira auffr une éclufe $\mathrm{EF}$ à lentrée du baffin inferieur dont les portes $G$ regardant la mer fe fermeront d'elles-mêmes quand elle montera, \& elle ne pourra entrer dans ce baflin uniquement deftiné à recevoir les caux d'en haur ; car le radier des mounlins étant à peu prés de niveau avec le lir du balfin fuperieur, l'eau pourra paffer de l'un dans l'autre, ar de-lí aller fe jetter à la mer, lorfque la marée en baiffant laifera ia liberté aux portes $G$. de s'ouvrir pour mettre ce baffin à fec de i hetres en 12 heures : or fil'on proportionne l'étendue de celuir d'en haut à la quantité d'cau que les moulins dépenferont pendant o on $\mathrm{o}$ heures, alin d'avoir ćgard au tems que la mer mettra à baiffer \& à remonter jufg̨u'à un certain point, les moulins iront continuellement fans aucune fur jettion.

Comme toutes les rivieres qui vont fe jetter à la mer ont un flux Plan. ?- 

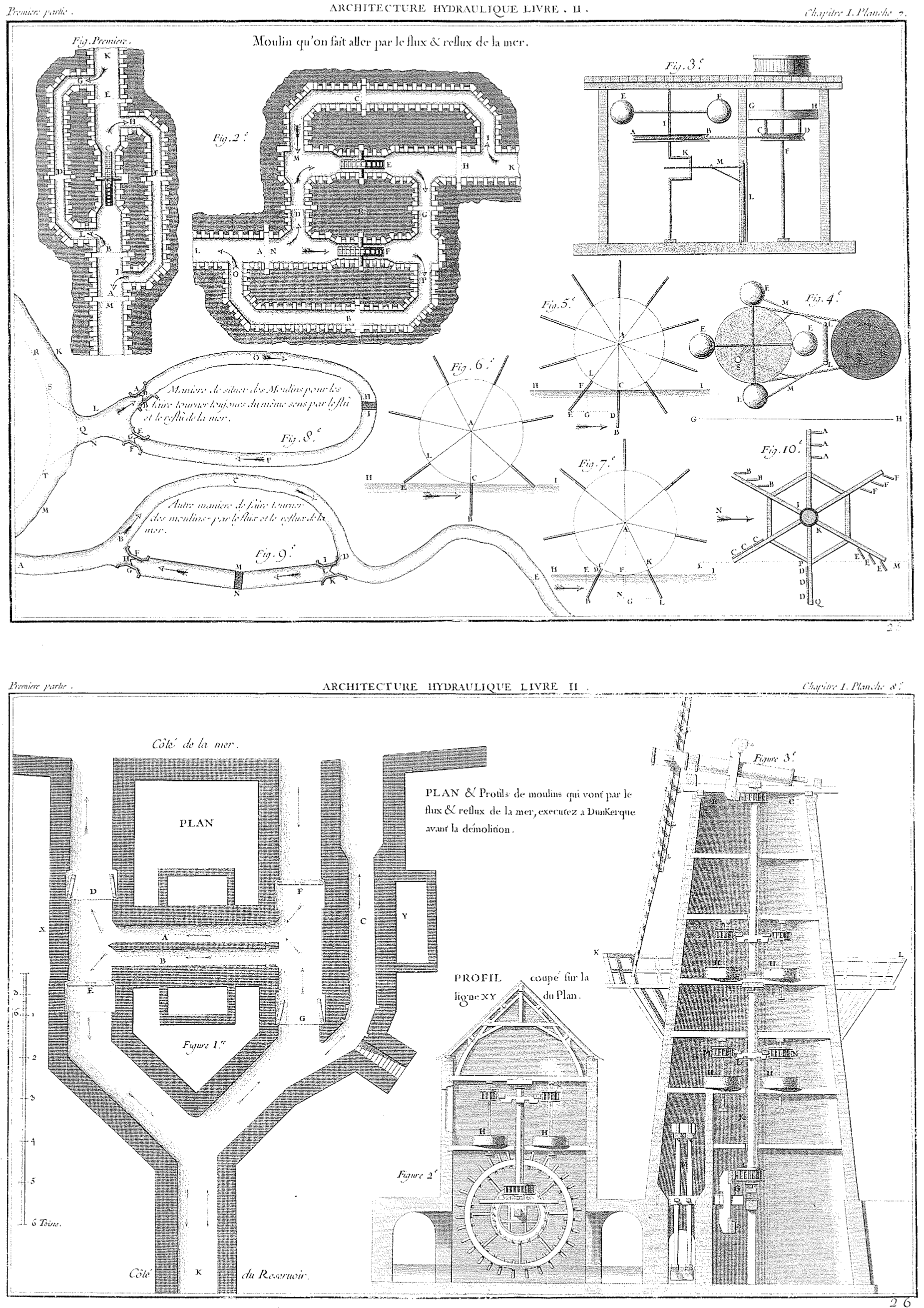

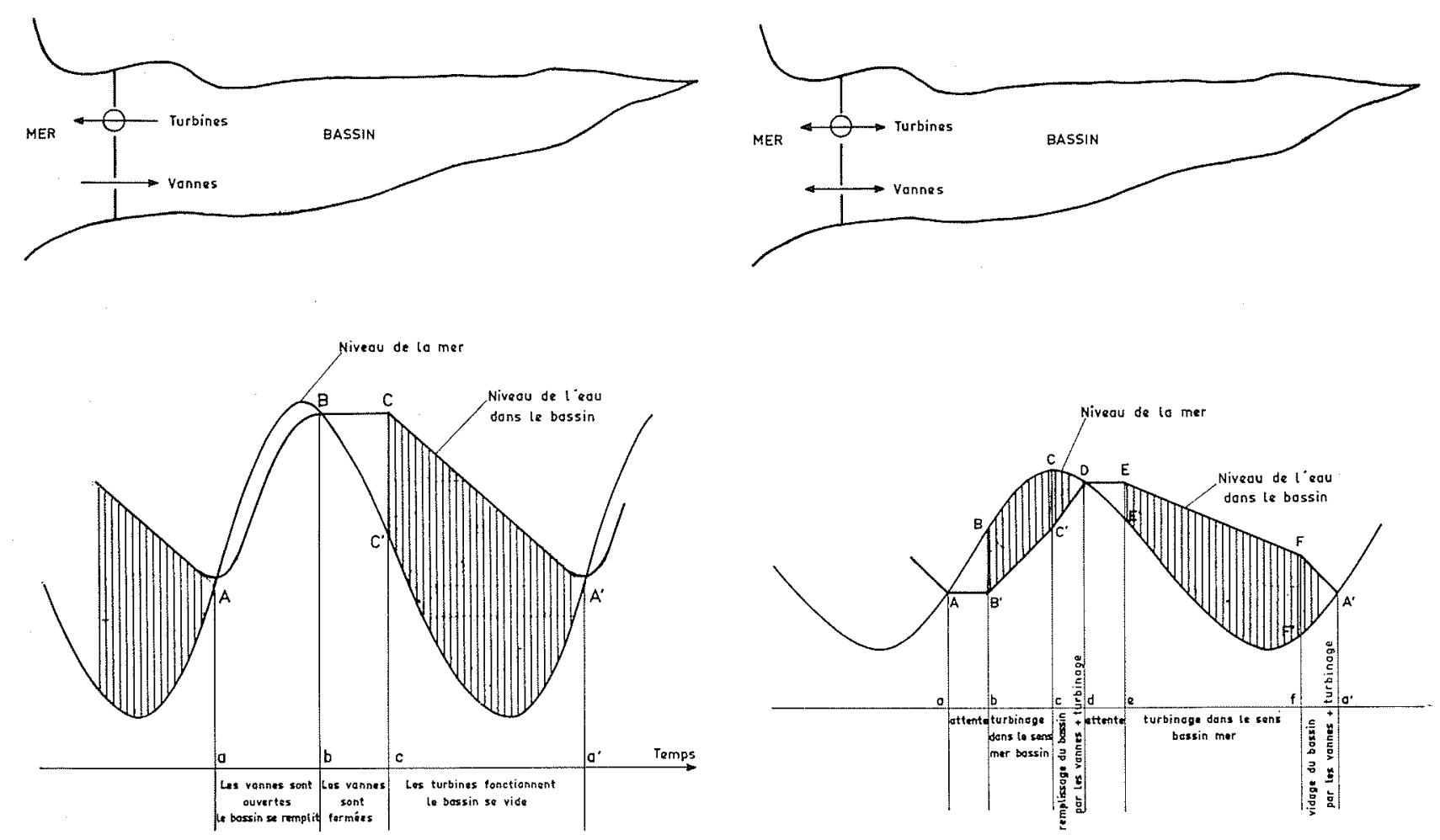

2/ Cycle à simple effet au vidage

3/ Cycle à double effet
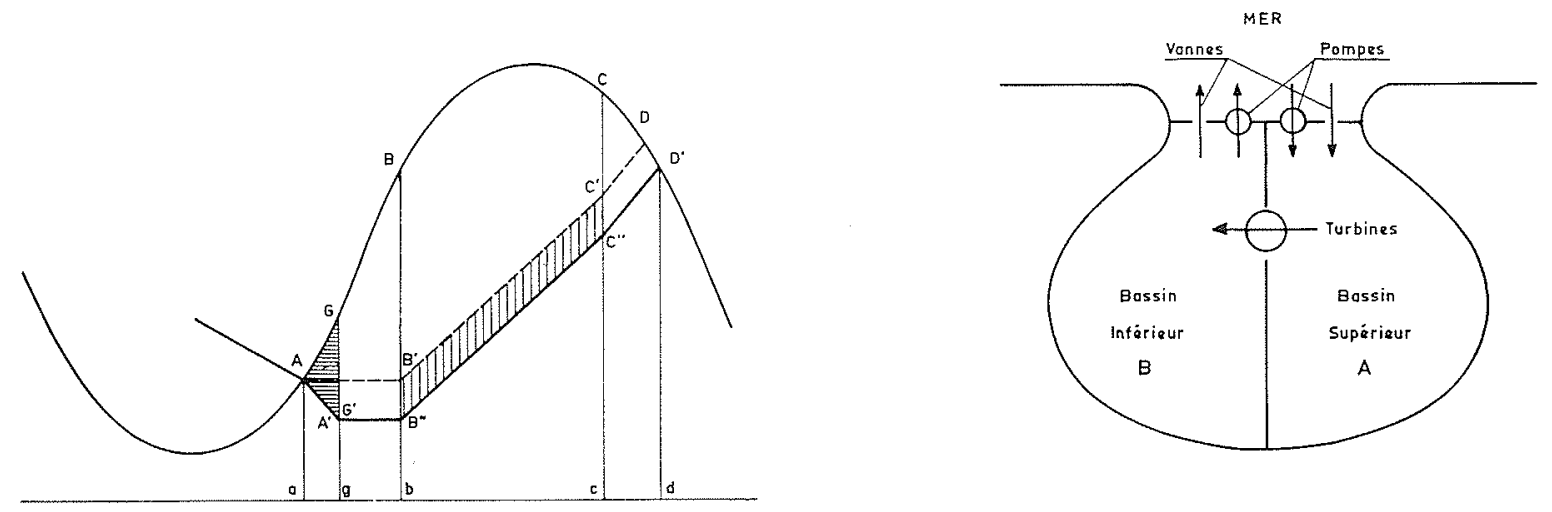

4 a/ Pompage avec sur-vidage du bassin ag : pompage - $g b$ : attente - $b c$ : turbinage

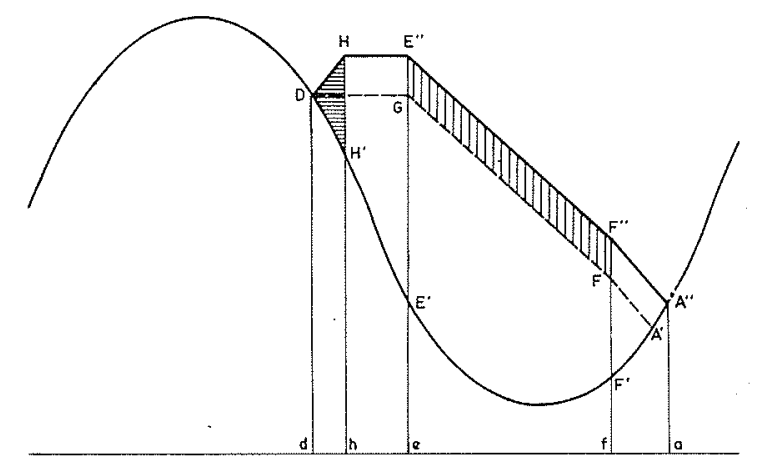

$4 \mathrm{~b} /$ Pompage avec sur-remplissage du bassin dh : pompage - he : attente - ef : turbinage

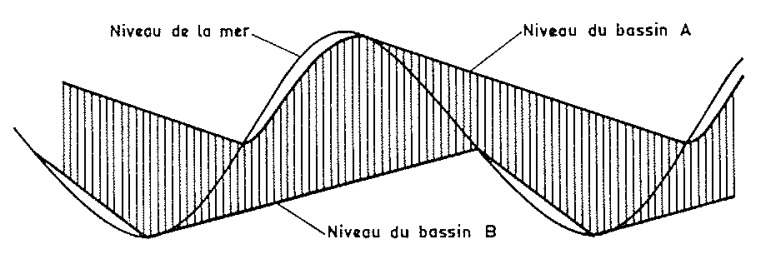

CYCLE SANS POMPAGE

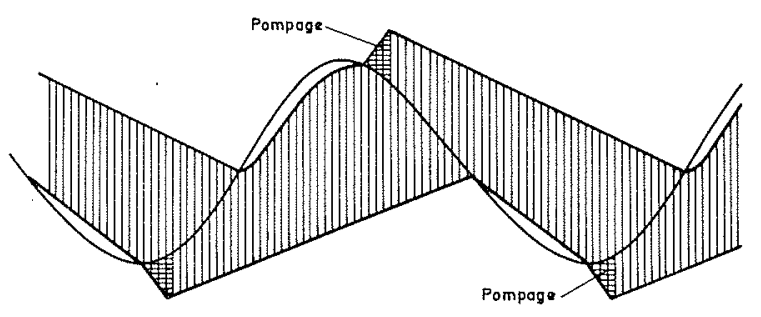

CYCLE AVEC POMPAGE

5/ Schéma de fonctionnement d'une usine marémotrice à deux bassins 
d'où l'énergie totale pour un cycle à double effet :

$$
E=\rho g A \int_{0}^{A} S(z) d z
$$

Désignons par $V$ le volume «marné » du bassin :

$$
E(\text { tonnes-poids } \times \mathrm{m})=A(\mathrm{~m}) . V\left(\mathrm{~m}^{3}\right)
$$

Une formule pratique peut en être déduite; elle donne l'énergie naturelle annuelle, en tenant compte de la répartition annuelle du coefficient de marée [7] (fig. 6) :

$$
E(\mathrm{GWh} / \mathrm{an})=S\left(\mathrm{~km}^{2}\right) A^{2}{ }_{100}(\mathrm{~m})
$$

avec :

- ${ }_{100}$ marnage de la marée de coefficient 100 ;

- $S$ surface du bassin à la cote de mi-marée.

Pour être applicable à des sites où le coefficient de marée n'a pas de sens, la formule doit être modifiée en introduisant le marnage moyen $A_{m}$ :

$$
E(\mathrm{GWh})=1,97 S\left(\mathrm{~km}^{2}\right) A_{m}^{2}(\mathrm{~m})
$$

Raisonnant ainsi nous commettons, selon l'image évoquée par R. Gibrat, l'erreur de croire déduire l'énergie disponible à une prise de courant en ne connaissant que l'amplitude de la tension alternative. En effet, en régime naturel, l'énergie dépensée par frottement sur le fond par les courants de marée est faible; l'énergie apportée par les ondes-marée est donc surtout réactive (par exemple capacitive au fond d'un golfe); le courant et la marée sont presque en quadrature; mais la présence d'une usine, qui appelle de la marée une énergie active, modifie le déphasage courant-marée: l'énergie provient d'ailleurs. Il apparaît ainsi la notion de perturbation apportée à la marée par une usine marémotrice. Dans cet ordre d'idée, la comparaison entre la puissance installée et la puissance active consommée par frottement sur le fond par l'onde-marée dans une zone donnée, est un indice de l'étendue du "remous extérieur " de l'usine. Par exemple, en vive-eau, environ $25 \mathrm{GW}$ sont consommés par frottement sur les fonds du golfe de Saint-Malo (à l'est de la ligne Bréhat-Guernesey-Aurigny-cap de la Hague); c'est beaucoup vis-à-vis des $0,24 \mathrm{GW}$ de la Rance, mais c'est du même ordre de grandeur que les puissances des projets Chausey (12 GW).

En définitive, la notion d'énergie naturelle n'est qu'un indice éventuellement utilisable dans le cas des usines de faible importance. Par exemple, l'énergie naturelle annuelle de la Rance est $2000 \mathrm{GWh}$; l'usine en produit le quart. Pour les projets plus grandioses, il convient d'abord d'estimer l'importance de la zone marine intéressée, par l'étude des transits d'énergie alentour, ensuite calculer le régime de marée avec l'usine en fonctionnement. Ces calculs, jadis faits en utilisant les modèles réduits physiques, sont à l'heure actuelle du ressort des ordinateurs.

\subsection{Les usines marémotrices dans le monde}

Les développements précédents montrent qu'un site marémoteur doit satisfaire aux critères fondamentaux suivants:

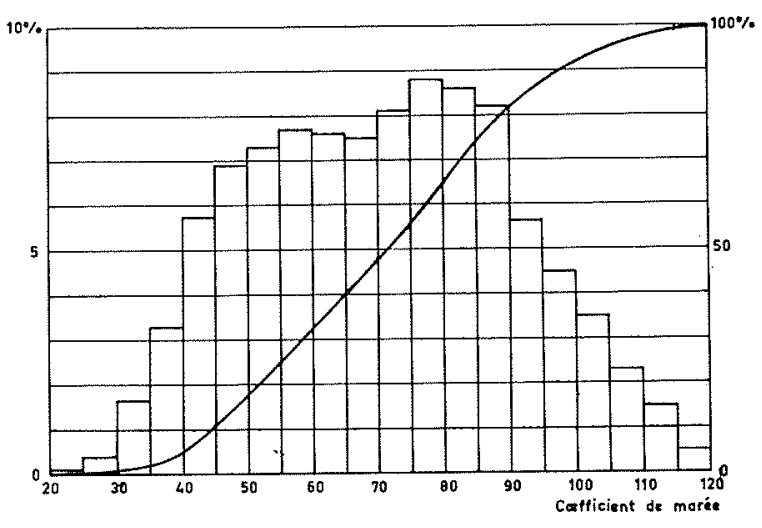

6/ Répartition des coefficients de marée le long des cótes trançaises (cycle 1935-1953)

- Ordonnées de gauche : pourcentage du temps où le coefficient est compris dans la gamme de 5 correspondante; par exemple le coefficient de marée est compris entre 65 et 70 pendant $7,5 \%$ du temps.

- Ordonnées de droite: pourcentage cumulé du temps où le coefficient est inférieur à une valeur donnée; par exemple pendant $65 \%$ du temps le coefficient est inférieur à 80 .

- marée de grande amplitude (au moins $5 \mathrm{~m}$ en moyenne);

— bassin à ouverture relativement étroite;

auxquels peuvent être ajoutées les considérations suivantes:

- profondeurs suffisantes pour pouvoir loger les turbines assez profondément;

- houle relativement faible;

- pas trop de risque d'ensablement;

- pas trop de gêne à la navigation;

- éviter les nuisances écologiques du fait du changement des niveaux de marée dans le bassin;

- éviter les nuisances économiques du fait du changement de rythme de marée dans le bassin.

Ces contraintes limitent les sites "possibles" dans le monde à une douzaine environ (cf. tableau 1 et fig. 1 et 7).

\subsubsection{Projet américain en Alaska [41e]}

Deux projets sont envisagés au fond du Cook Inlet près d'Anchorage. Le projet Knik Arm a fait l'objet d'une estimation économique en 1970 conduisant à une dépense d'investissement de 640 millions de dollars et un coût de $1,2 \cdot 10^{-3} \$$ par $\mathrm{kWh}$ pour $1 \%$ d'intérêt (soit environ $6 \mathrm{c} / \mathrm{kWh}$ pour un intérêt de $10 \%$ ).

\subsubsection{Projet U.S.A.-Canada de Passamaquoddy}

A l'occasion du New Deal (1935) le président Roosevelt fit étudier un projet de $210 \mathrm{MW}$, qui fut abandonné l'année suivante lors de la révision des crédits de lutte contre le chômage. Le projet fut reconsidéré à plusieurs reprises; la dernière étude de 1964 concerne deux usines de $1 \mathrm{GW}$ entre deux bassins fonctionnant en pointe, en connexion avec une usine hydraulique de $750 \mathrm{MW}$. Mais les marées étant faibles (maximum $7,5 \mathrm{~m}$ ) le projet ne paraît pas économiquement valable. 


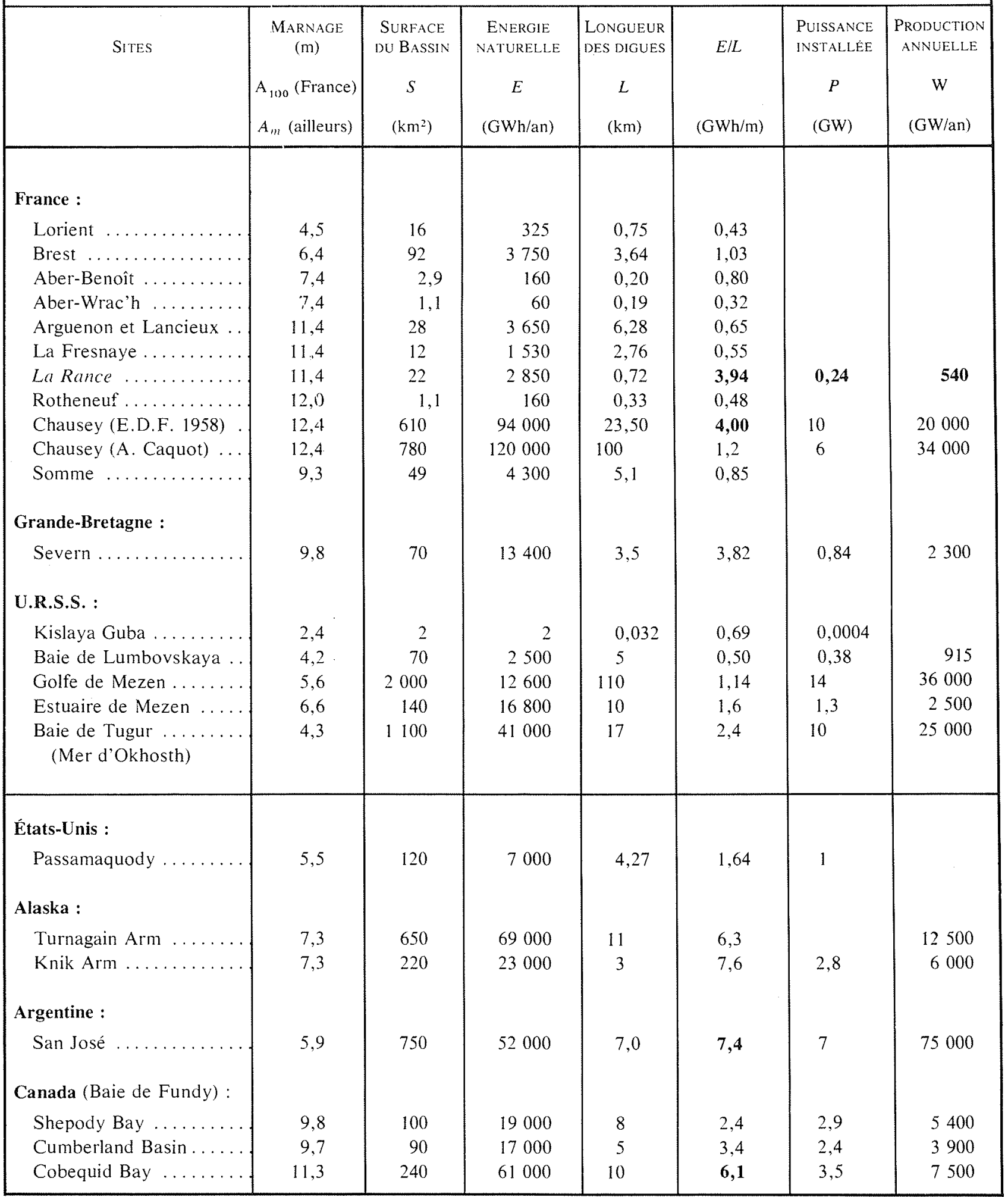




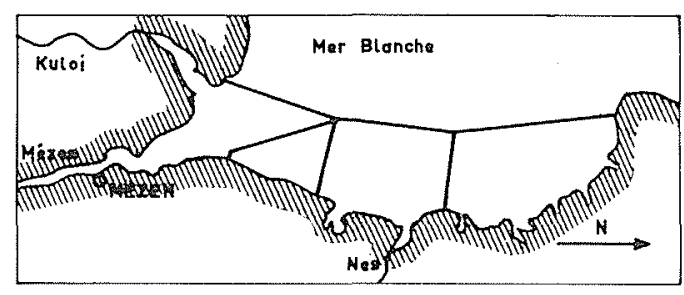

Golfe de Mezzen

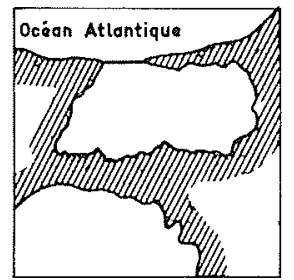

Golfe de Soint José

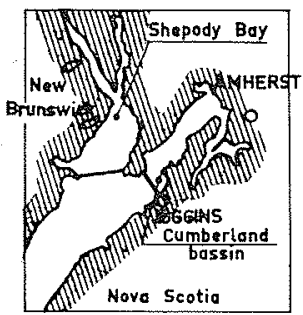

Boje de Fundy

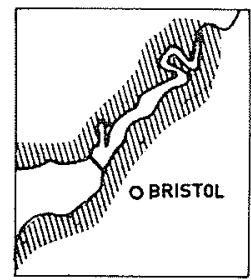

Lo Sévern

Echeile:

$20 \mathrm{~km}$

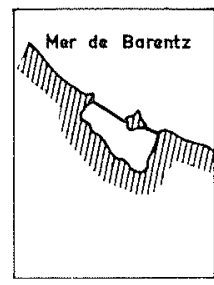

Lumbovskya

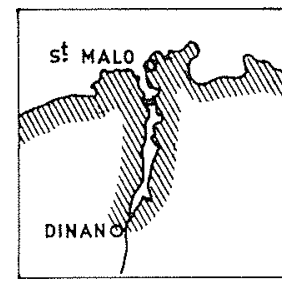

La Rance

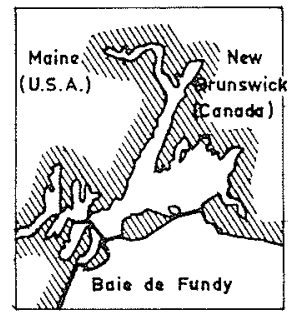

Golfe de Passamaquoddy

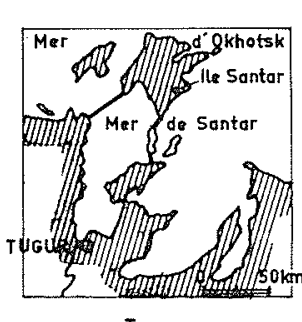

Tugur

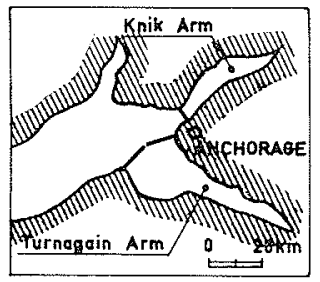

Cook Inlet

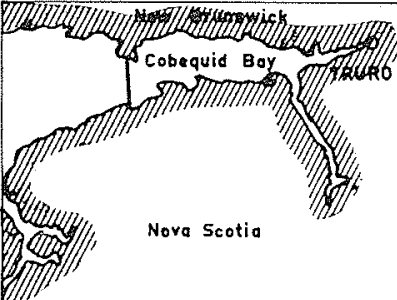

Boie de Fundy

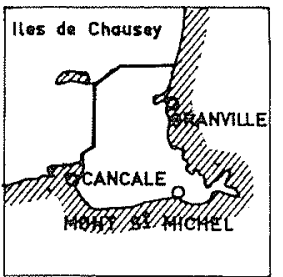

Chousey
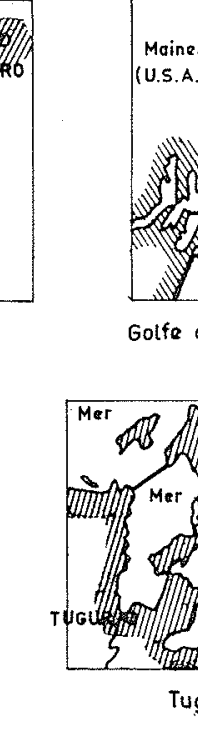

7/ Les grands sites marémoteurs

\subsubsection{Projet canadien de la baie de Fundy}

Il s'agit d'équiper les estuaires débouchant dans une zone à très fort marnage ( $16 \mathrm{~m}$ en vive-eau). Trois projets, dont un de $3,5 \mathrm{GW}$ avec groupes bulbes de $40 \mathrm{MW}$, ont été examinés de 1966 à 1969. Compte tenu de la situation économique de l'époque, ils furent abandonnés. Le prix du projet le plus important était estimé à 720 millions de dollars en 1968, soit une dépense annuelle de 56 millions de dollars à $7 \%$, soit environ $3,7 \mathrm{c} / \mathrm{kWh}[41 a, 41 b, 46]$. Une analyse détaillée du projet a été publiée par R. Gibrat [40] à partir du rapport canadien [39]. Il est intéressant d'en résumer les conclusions en énumérant les conditions de réalisation du projet : abaissement du taux d'intérêt de l'argent (3 à $4 \%$ ), réduction du prix du génie civil, augmentation du coût des autres énergies du fait de la pollution, épuisement des sources d'énergie plus économiques [103].

\subsubsection{Projets soviétiques}

Une seule usine expérimentale, équipée d'un groupe français de $400 \mathrm{~kW}$, fonctionne depuis 1968 à Kislaya Guba, à $60 \mathrm{~km}$ au nord de Murmansk sur la mer de Barentz. Il s'agit d'une usine-caisson préfabriquée, amenée par flottaison, longue de $36 \mathrm{~m}$, large de $18,3 \mathrm{~m}$, haute de $15,35 \mathrm{~m}$, pouvant contenir deux groupes bulbes de diamètre $3,3 \mathrm{~m}$. Deux grands projets $(1,3$ et $14 \mathrm{GW}$ ) en mer Blanche (golfe de Mezen) furent étudiés en 1961 sous l'impulsion de L. Bernstein [30]; actuellement un nouveau projet de $10 \mathrm{GW}$ est envisagé en mer d'Okhotsk [41 l, 45],

\subsubsection{Projet anglais de la Severn}

Le premier projet, étudié de 1918 à 1933, fut abandonné par suite du faible prix du charbon; il fut repris 
en $1955(800000 \mathrm{~kW}$ installés, 2,3 TWh par an, marnage de $14,5 \mathrm{~m}$ ), puis abandonné pour raisons économiques.

\subsubsection{Projets français}

Une dizaine de sites ont été étudiés en France [101]; le projet de l'Aber-Wrac'h fit même l'objet d'un commencement d'exécution en 1928. Le tableau 1 montre que, finalement, est réalisé le projet où le rapport $E / L$ ( $L$ étant la longueur du barrage) est le plus important; ce tableau montre en outre la suprématie des projets Rance et Chausey (E.D.F.) sur tous les autres projets.

Tous les abandons de projets (y compris Chausey E.D.F.) ont eu lieu en 1966; ils avaient pour cause la baisse du prix du fuel. Mais il est intéressant de savoir que, pour tous les projets, le paramètre défavorable le plus important est le taux d'intérêt; dans les contextes énergétiques d'avant 1966, la rentabilité des capitaux investis dans la construction des usines marémotrices exigeait un intérêt de l'ordre de 3 à $4 \%$. Ceci montre bien que le "sort » des usines marémotrices est lié aux contextes politique et psychologique qui affectent le loyer de l'argent. Les problèmes scientifiques et technologiques, que nous évoquerons en décrivant les projets français de la Rance et de Chausey, et qui sont déjà en grande partie résolus, sont en regard secondaires.

\section{La Rance}

\subsection{Historique}

La naissance de l'idée de la Rance est admirablement contée dans l'avant-propos de l'ouvrage l'Énergie des marées de R. Gibrat [8].

En 1920, l'Ingénieur en Chef des Ponts et Chaussées à Rennes, M. Boisnier écrivait un rapport sur l'utilisation de l'énergie des marées dans la Rance. Enthousiasmé pour cette idée, R. Gibrat fondait en 1943 la Société d'Etudes pour l'Utilisation des Marées (S.E.U.M.), qui, à la nationalisation d'Electricité de France en 1946, devenait Service avec les mêmes initiales. Animé par L. Vantroys, ce Service élaborait un avant-projet, suffisamment mûri pour, qu'en 1951, une Région d'Equipement d'E.D.F. fût chargée des études de réalisation et que, le 25 octobre 1952, le Président de la République, lors de l'inauguration de l'usine de Donzère-Mondragon, annonçât l'aménagement prochain de l'estuaire de la Rance.

Passons sur les vicissitudes politiques et économiques pour ne retenir que quelques dates:

- 29 décembre 1960, le Ministre de l'Industrie autorisait E.D.F. à engager un programme d'aménagement hydroélectrique comprenant la Rance;

- les 10 et 11 janvier 1961, E.D.F. notifiait, au groupement d'entreprises "Tramarance», un marché de 32 milliards d'AF (environ $580 \mathrm{MF}$ 1974), et au groupement des constructeurs des groupes de la Rance la commande des 24 groupes bulbes marémoteurs ;
- le 20 juillet 1963 , à $10 \mathrm{~h}$ la coupure était terminée : l'estuaire était séparé de la mer;

- le 26 novembre 1966, le Président de la République inaugurait l'usine;

- le 4 décembre 1967, le $24^{*}$ groupe était mis en service.

\subsection{Le site}

«La Rance est un petit fleuve breton, de $100 \mathrm{~km}$ de longueur, au débit insignifiant, qui, après son passage au pied des remparts de la cité féodale de Dinan, débouche dans une large vallée que la mer visite et inonde deux fois par jour ". Cette phrase de G. Mauboussin $[41 c]$ est une excellente description de l'estuaire

Le site a toujours tenté les ingénieurs; des projets furent établis en 1897, 1902, 1907; en 1918, Maire demanda la concession de l'estuaire pour y installer une usine de $290000 \mathrm{VA}$ [3], dont la construction était estimée à 26 millions de $F$ et l'exploitation à 3 millions de F par an. Le projet E.D.F. de 1951 comportait 26 groupes de $8000 \mathrm{~kW}$ équipés de turbines Kaplan classiques à axe vertical; il était trop onéreux. Dix ans plus tard le projet de 1960 devenait économiquement valable, en grande partie grâce à l'emploi des groupes bulbes.

L'estuaire de la Rance, comporte une série d'étranglements favorables à l'édification d'un barrage (fig. 8). Le site choisi est le resserrement des rives entre les pointes de la Brebis et de la Briantais, assez près de la mer pour accroître la superficie du bassin, mais assez loin des parages fréquentés de Saint-Servan pour que les courants dus à l'usine ne gênent pas la navigation.

Le problème hydraulique fut ardu: il s'agissait d'arrêter un débit alternatif atteignant $18000 \mathrm{~m}^{3} / \mathrm{s}$ en vive-eau. L'usine, à $4 \mathrm{~km}$ de l'embouchure de l'estuaire, est relativement à l'abri de la houle; cependant, par tempête de $\mathrm{N}-\mathrm{W}$, une hauteur de houle de 2,6 $\mathrm{m}$ fut enregistrée au niveau du barrage.

\subsection{Réalisation de l'usine}

\subsubsection{Les études théoriques du S.E.U.M.}

Dès 1947, l'équipe du S.E.U.M. s'attaque au problème énergétique : la définition, le classement, le calcul des cycles de fonctionnement. Il s'agit d'une action originale essentiellement théorique, où tout est innovation; elle fait apparaître l'intérêt du pompage qui permet de rentabiliser l'énergie disponible en heure creuse; elle montre qu'une usine marémotrice est un aménagement hybride entre un réservoir et une usine au fil de l'eau; mais elle ne lève pas l'ambiguité entre usine de pointe et usine de base. Elle fait aussi apparaitre les points faibles : le remous, c'est-à-dire la diminution de la chute due à la rétention de l'eau dans le réservoir, et l'effet perturbateur de l'usine sur la marée; ce point a peu d'influence dans le cas de la Rance, mais son rôle serait important pour des usines de très forte puissance marémotrices ou non.

La partie théorique évoquée ci-dessus est à la base de la décision de construire l'usine, mais elle n'a pas débouché sur une solution satisfaisante du point de vue 
pratique. De ce fait, l'organisme responsable de l'exploitation, le Centre Interrégional des Mouvements d'Energie de l'Ouest, utilise un outil appelé «programmation dynamique » sur lequel nous reviendrons très en détail.

Parallèlement une équipe d'études sur le site s'installait à Saint-Servan sous la direction de Pierre Courcambeck. On lui doit :

- d'avoir complété les travaux d'hydrographie et de courantologie ;

- les recherches sédimentologiques et géologiques du site :

- les études de "corrosion » qui à elles seules constituent une des plus importantes «retombées» de la Rance ;

- enfin les études sur les bétons à la mer.

\subsubsection{Les modèles réduits}

Les modèles physiques ont apporté une importante contribution, puisque le problème comportait un large volet hydraulique. Un premier modèle fut construit, au Laboratoire Dauphinois d'Hydraulique à Grenoble, dès 1944, aux échelles de 1/500 en plan et 1/80 en hauteur; il servit surtout à l'élaboration des divers programmes proposés pour la réalisation de l'ouvrage. Le deuxième modèle, confié au Laboratoire National d'Hydraulique de Chatọu, fut construit en 1954, au 1/150, à SaintServan à proximité du site, ceci afin de simplifier la coordination des études sur modèle et sur le chantier, surtout en prévision des phases cruciales de la coupure. Cette décision fut des plus heureuses; elle a soudé les volontés d'organismes divers; elle a eu un grand retentissement sur le plan humain, permettant la réalisation d'efforts soutenus, en particulier lors des études de coupure qui duraient plusieurs jours sans arrêt [29a].

Le modèle de Saint-Servan fut surtout l'outil de mise au point de détail des opérations de coupure de l'estuaire, c'est-à-dire :

- le projet d'appel d'offre de 1955 de la Région d'Equipement Hydraulique ${ }^{\circ} 8$;

- les onze variantes proposées par les entreprises soumissionnaires ;

- enfin le projet 1960 effectivement réalisé en nature.

Il permit en outre la détermination des effets hydrauliques de l'usine :

- son remous pour les différents cycles d'exploitation, résultats dont tient compte le programme actuel de marche de l'usine;

- les conditions de navigation dans l'estuaire, études à la base des consignes de navigation;

- les travaux permettant une meilleure alimentation hydraulique des groupes et des vannes (implantation, situation et formes des pertuis de vannage, profils hydrauliques des ouvrages, déroctages, etc.);

- la détermination des intumescences dues aux variations du fonctionnement de l'usine: disjonction, arrêts, démarrage, etc. ;

- les effets sur le nouveau cheminement des effluents des égouts de Dinard et Saint-Servan.

En ce qui concerne l'étude des méthodes de coupure de l'estuaire, le modèle fut surtout l'outil de prédiction

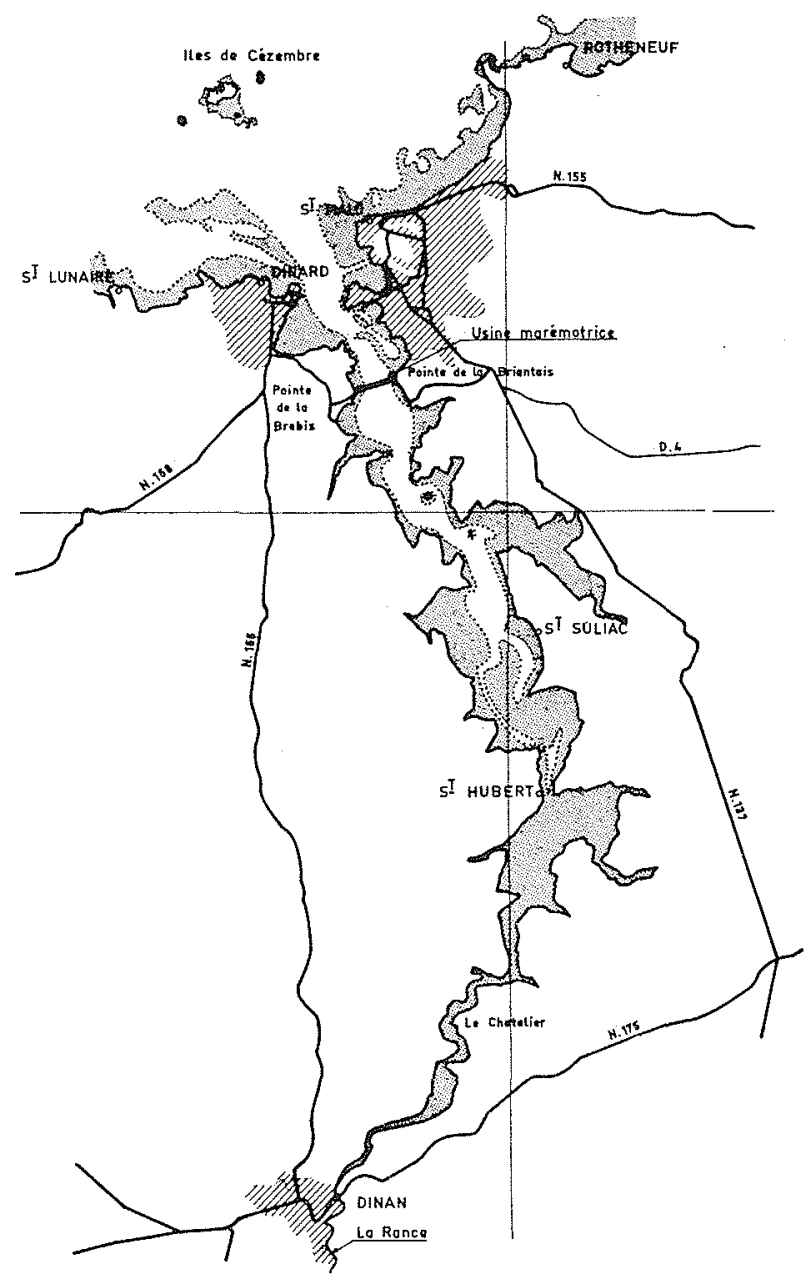

8/

des conditions hydrauliques (dénivellation de part et d'autre des ouvrages, courants, durées des étales,...) pour chaque situation de chantier, puis l'outil de calcul des efforts hydrauliques sur les ouvrages.

Ce modèle a permis au Laboratoire National d'Hydraulique de terminer la mise au point de ses matériels de reproduction de la marée, puis de sa télémesure (vitesse et niveau) et enfin d'extensométrie. Il faut retenir de l'étude sur modèle, à titre d'exemple, deux choix judicieux : l'emplacement sur le site et l'échelle $1 / 150$ sans distorsion, excellente pour l'étude des projets. En revanche, la limite maritime du modèle était trop proche de l'usine; le remous côté mer ne put donc être étudié. Le modèle n'aurait pas permis d'aborder les problèmes sédimentologiques; mais ceux-ci ne se sont pas posés.

\subsubsection{Les travaux en bureau d'études}

Mais les modèles réduits ne devaient leur succès spectaculaire (la visite du modèle réduit de la Rance à Saint-Servan figurait sur les circuits touristiques malouins) que parce qu'ils concrétisaient et confirmaient les travaux des bureaux d'études. La Région d'Equipement Hydraulique $n^{\circ} 8$ élaborait d'abord un projet d'ap- 


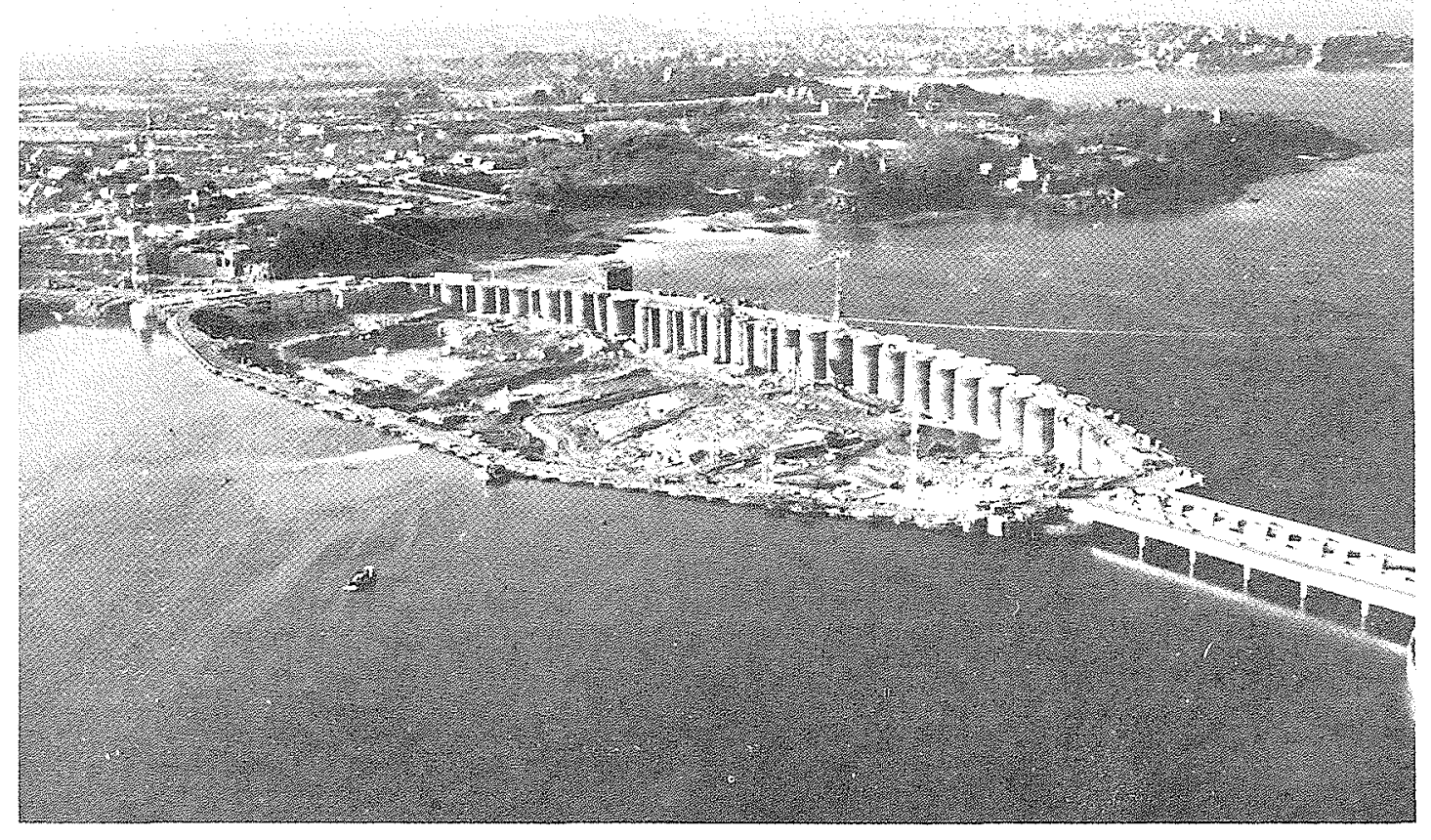

9 a/ Vue générale du batardeau de fermeture de l'estuaire de la Rance

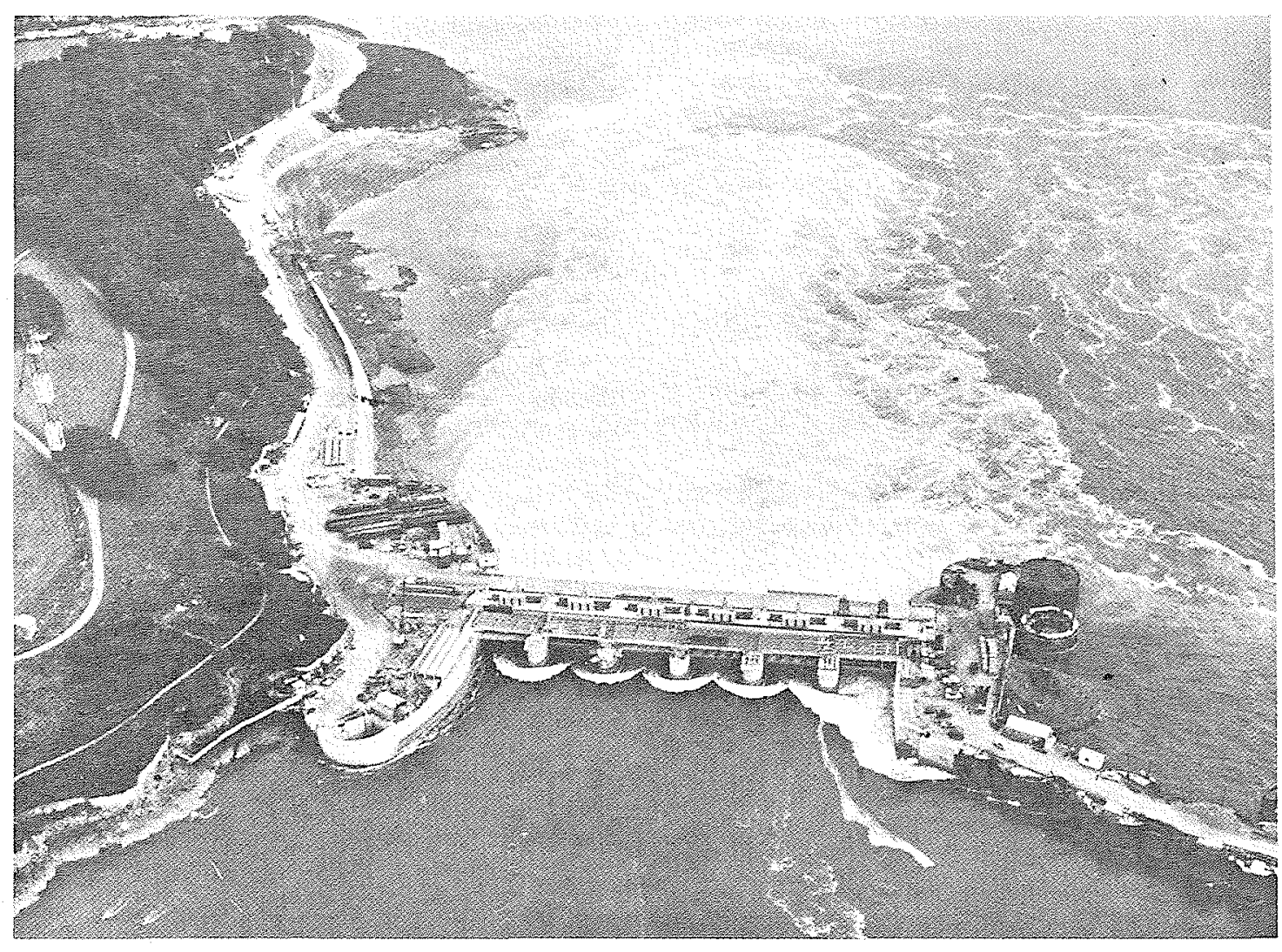

9 b/ Ecoulement par les pertuis lors dę la construction de l'usine de la Rance 
pel d'offre, puis aboutissait enfin au projet de 1960 , tenant compte des idées ingénieuses de $\mathrm{A}$. Caquot et des ingénieurs de "Tramarance", le groupement d'entreprises titulaires du marché. L'historique des projets retrace l'évolution des idées, depuis les projets de coupure par digues en enrochements, par caissons préfabriqués échoués sur des massifs d'enrochements, et jusqu'aux gabions circulaires en palplanches.

La solution finale utilise successivement :

- une digue en enrochements entre la pointe de la Briantais et l'îlot de Chalibert, pour servir d'abri à l'édification des gabions;
- des gabions jusqu'à limite du possible de part et d'autre de l'estuaire;

- des caissons préfabriqués échoués sur le fond au centre de l'estuaire, et consolidés par des gabions (fig. 9).

Le résultat des travaux de bureau d'études c'est aussi l'évolution entre l'usine à 40 groupes de 1955 et l'usine à 24 groupes de 1960. Les figures 10 et 11 illustrent la réduction de l'importance des ouvrages, donc l'effort de recherches technologiques dans un but évident d'économie sans nuire à la qualité de l'ouvrage.

Puisque la coupure fut réussie, le devis (420 MF 1960)

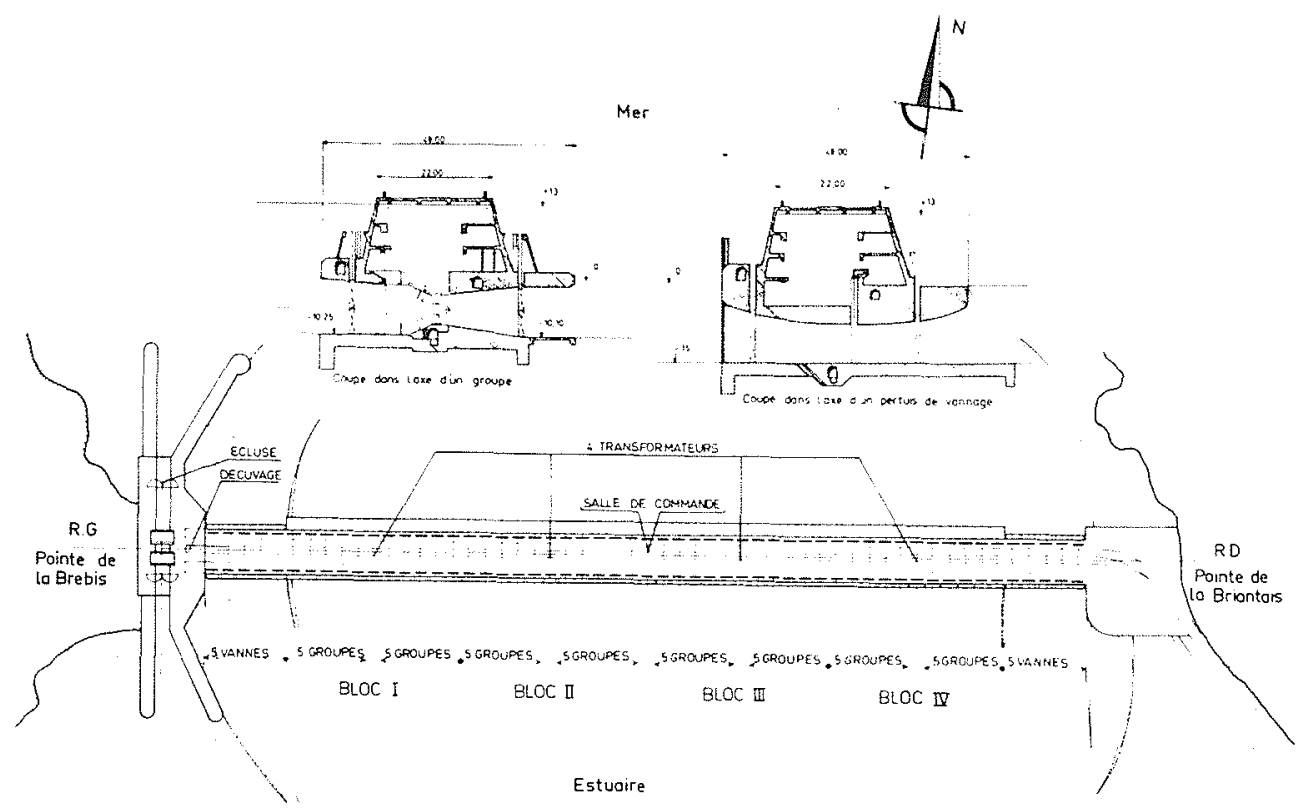

LE PROJET D'APPEL D'OFFRES A 40 GROUPES

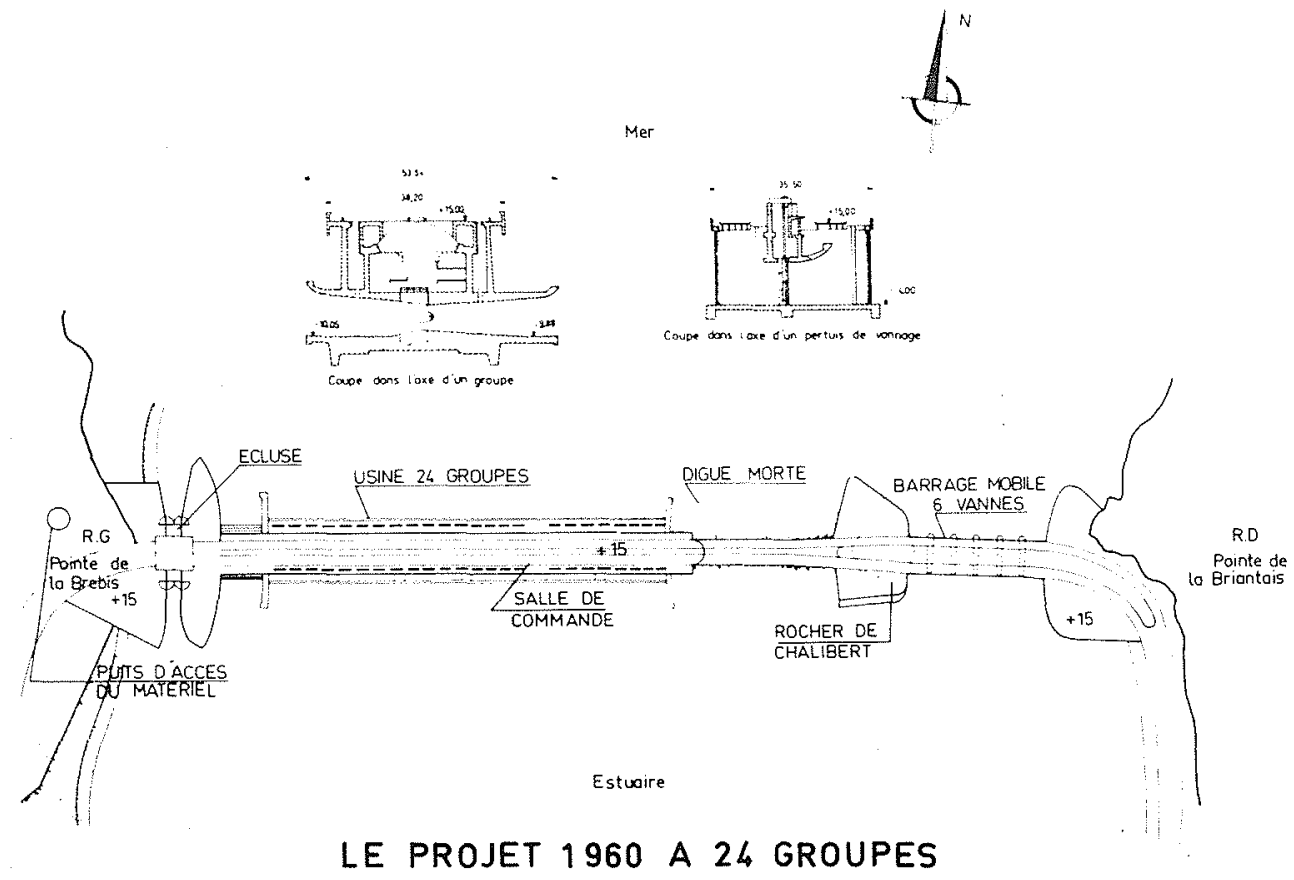

10/ L'évolution des projets de l'usine de la Rance de 1955 à 1960 


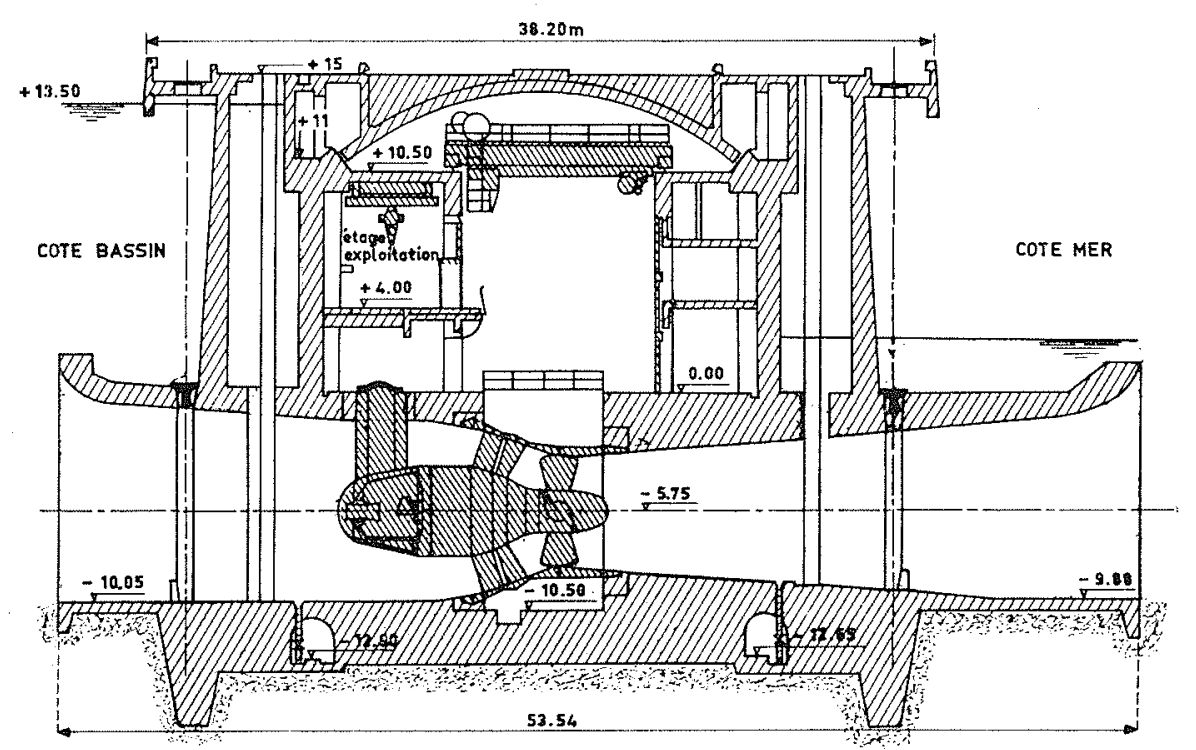

11 a/ Coupe par une travée de groupe

Cotes rapportées au zéro des cartes marines

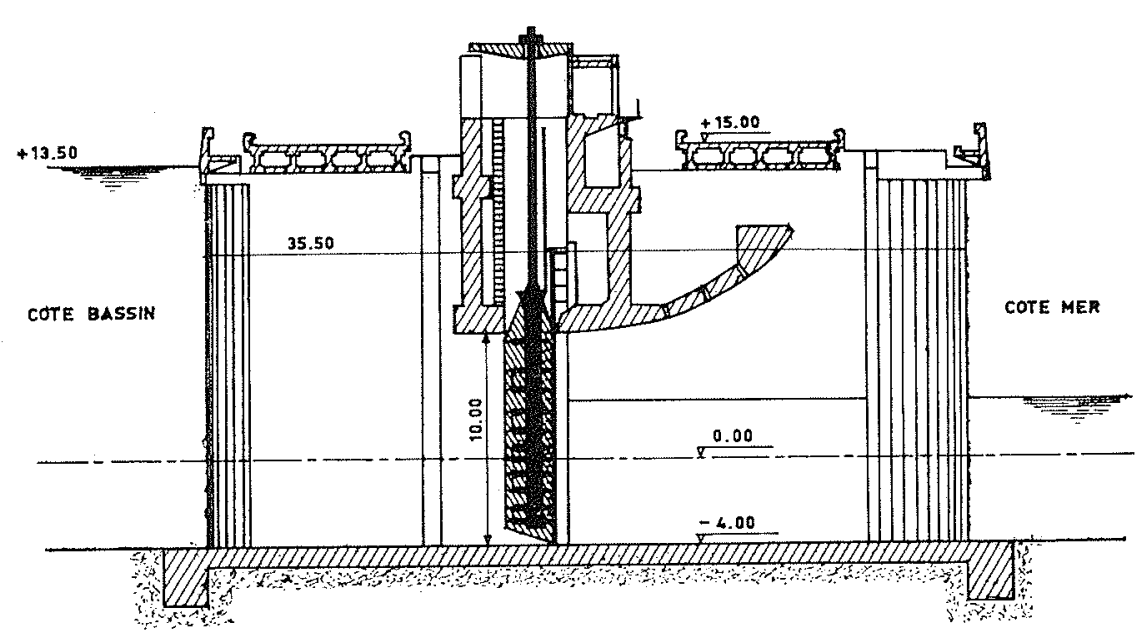

$11 \mathrm{~b} /$ Coupe par un pertuis de vanne
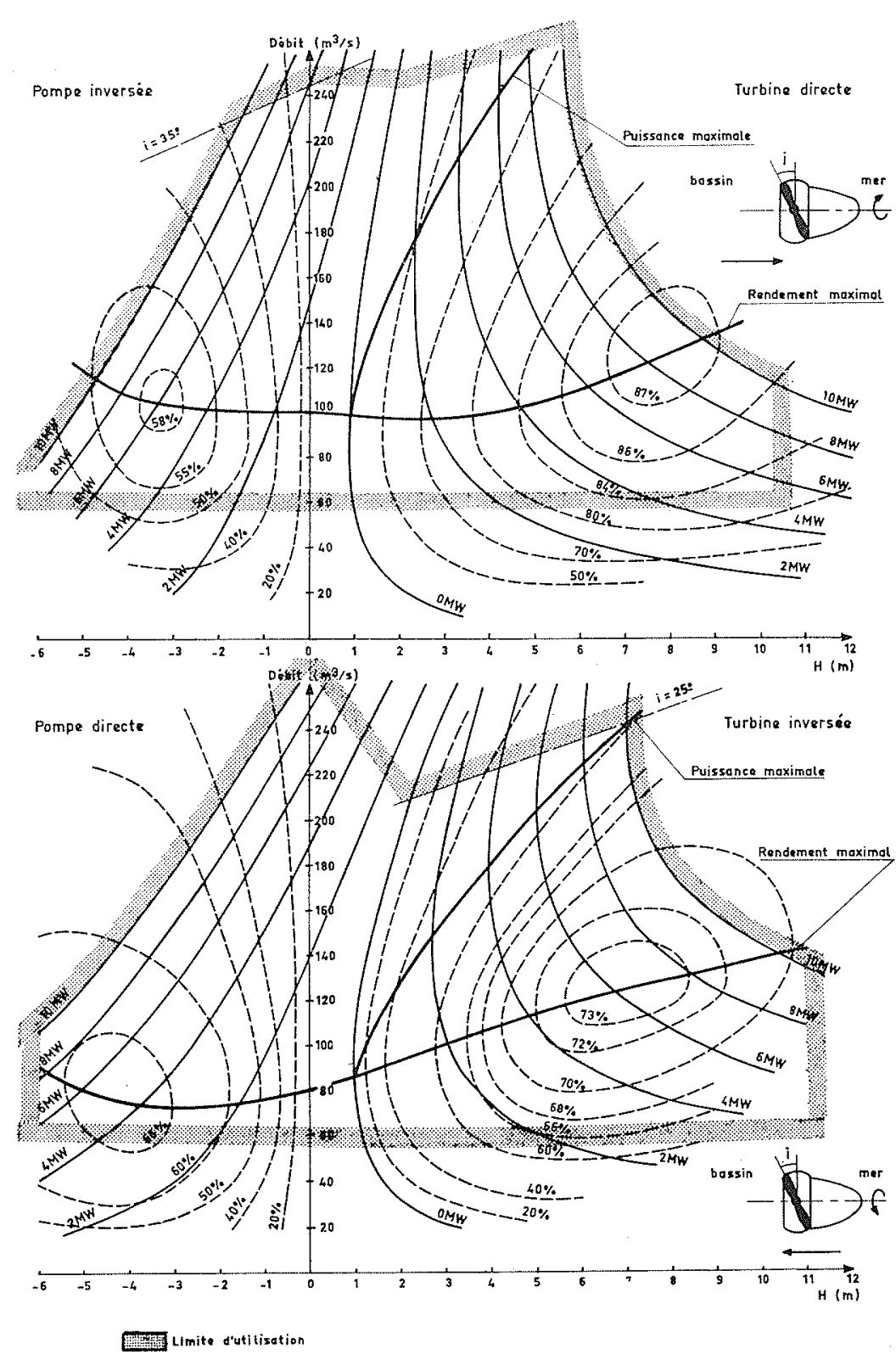

12/ Caractéristiques des groupes de la Rance 
respecté à $12 \%$ près (470 MF 1960), et l'usine réalisée dans les délais à quelques mois près, on peut se féliciter de la méthode de coupure choisie le 24 mai 1957. En revanche, que penser de la décision de juger insuffisante la rentabilité marginale de 16 groupes supplémentaires? la réponse à cette question est à chercher dans l'analyse du fonctionnement de l'usine; nous verrons que la Rance semble sous-équipée en vive-eau.

\subsection{Les groupes bulbes}

\subsubsection{Historique}

Le groupe bulbe fait partie de la classe des turbomachines à écoulement axial; l'eau emprunte un parcours rectiligne suivant l'axe de rotation de la machine; la machine électrique est généralement entièrement immergée. L'idée remonte à 1925 (brevet américain Harza); la première réalisation date de 1936 sous forme d'un groupe de $195 \mathrm{~kW}$ à Röstin (Poméranie). Les difficultés de la technique appliquée à ces groupes (condensation de l'humidité et entretien d'un joint d'eau entre les parties fixes et tournantes) furent surmontées, mais sans laisser espérer de pouvoir dépasser $2 \mathrm{MW}$ de puissance pour les groupes exécutés par Arno-Fischer pour l'équipement du Lesets et de l'Iller en Bavière [44f].

En France, dès 1943, la S.E.U.M. étudiait avec Neyrpic un bulbe de $20 \mathrm{MW}$, mais reculait devant les difficultés; en particulier, le diamètre de l'alternateur (bulbe de $12 \mathrm{~m}$ ) était beaucoup plus grand que celui de la roue $(8 \mathrm{~m})$. Plus tard, en 1950 , la S.N.C.F. décidait d'équiper la chute de Castet de groupes asynchrones de $810 \mathrm{~kW}$; E.D.F. mettait au point des groupes immergés expérimentaux pour les micro-centrales synchrones ou asynchrones de $160 \mathrm{~kW}$ (la Mayenne), $52 \mathrm{~kW}$ (Echarcon), $200 \mathrm{~kW}$ (Rethel) et $800 \mathrm{~kW}$ (Lartige).

Parallèlement à ces recherches pour l'équipement de basses chutes en rivière, E.D.F., avec plusieurs constructeurs, poursuivait les études sur les groupes bulbes industriels $[11 f]$, études qui furent suivies par les décisions d'équiper Cambeyrac (2 groupes de $5 \mathrm{MW}$, 1957), Argentat (2 groupes de $14 \mathrm{MW}, 1958$ ) et Beaumont-Monteux (8:5 MW, 1959). Le site d'Argentat permit d'étudier le simple effet; il montra l'intérêt du fonctionnement de l'alternateur dans l'air comprimé pour évacuer la chaleur (solution adoptée à la Rance sous la pression de 2 bars). L'installation de Cambeyrac, conçue pour étudier le double effet, mit en relief les difficultés de retourner les pales ou d'utiliser deux roues dont l'une est immobilisée comme distributeur. Les essais montrèrent de plus qu'il y a intérêt, du point de vue rendement, à disposer à l'amont le corps du bulbe contenant l'alternateur; le bulbe, placé dans le conduit hydraulique avant la roue, perturbe alors moins l'écoulement que s'il est dans le diffuseur; la figure 12 illustre ce fait en montrant les différences de rendement en turbine directe et inversée.

\subsubsection{Le groupe expérimental de Saint-Malo}

La mise en service d'un groupe expérimental installé dans une écluse désaffectée du port de Saint-Malo, marque le début de la deuxième étape, celle des grou- pes à pales non réversibles. Avec la vérification en grandeur nature des résultats des études de rendement sur modèle réduit, et la confirmation de la possibilité de l'évacuation de la chaleur par l'eau de circulation, cette étape vise à soumettre le matériel à l'épreuve de l'eau de mer.

Les caractéristiques du groupe étaient les suivantes:

- puissance $9 \mathrm{MW}$

- débit $300 \mathrm{~m}^{3} / \mathrm{s}$;

- diamètre de la roue $5,8 \mathrm{~m}$;

— incidence des pales de -- 10 à $+35^{\circ}$;

- vitesse de rotation $88,30 \mathrm{tr} / \mathrm{mn}$.

La grande nouveauté de ce groupe est le dessin des pales en forme d'S très allongé, tel que la cambrure du bord d'attaque soit correcte dans tous les cas, et permettant, lorsque la chute change de sens, le passage du fonctionnement turbine à celui pompe pour le même sens d'écoulement de l'eau. En outre les groupes arrêtés peuvent servir de vanne.

De 1959 à 1964, le groupe expérimental fut soumis à une exploitation sévère $(1678 \mathrm{~h}$ de marche, 2483 démarrages). Les essais ont confirmé les excellentes valeurs attendues du rendement $[27 b]$; ils ont permis d'analyser les conditions de stabilité et de définir les zones à rigidifier pour supprimer les vibrations; en fait ces zones seront peu sollicitées du fait de la rareté des démarrages en moteur asynchrone, puisque la machine passe continument du fonctionnement en turbine au fonctionnement en pompe [27a].

\subsubsection{Les groupes de la Rance}

Outre l'effet psychologique levant certains doutes subsistant sur la fiabilité des groupes immergés, le bon fonctionnement et les résultats concrets du groupe de Saint-Malo, ont fait adopter les caractéristiques suivantes pour les groupes de la Rance :

- puissance $10 \mathrm{MW}$;

- débit $250 \mathrm{~m}^{3} / \mathrm{s}$;

- diamètre de la roue $5,35 \mathrm{~m}$;

- incidence des pales de -5 à $+35^{\circ}$;

— vitesse de rotation $93,75 \mathrm{tr} / \mathrm{mn}$.

Le groupe est plus " compact "; le diamètre du bulbe passe de 5 à $4,38 \mathrm{~m}$, ce qui améliore les qualités de fonctionnements en «inversé »; le groupe est plus court, le coût du génie civil en est réduit. Avec cette machine, la technique du bulbe trouve son épanouisse-

ment; la porte est ouverte à la réalisation des groupes de grande puissance (20 MW à Pierre-Bénite, $35 \mathrm{MW}$ à Beaucaire, etc.).

\subsubsection{L'évolution des groupes bulbes}

Quel est l'avenir des groupes bulbes? Des unités de $50 \mathrm{MW}$ avec roue de $7,5 \mathrm{~m}$ sont en projet. Le problème du porte-à-faux de la roue, source d'efforts importants sur les paliers et de vibrations, est maintenant résolu; les groupes bulbes ne vibrent plus; ceci est corroboré par le fait que des groupes français de $54 \mathrm{MW}$ sont livrés aux U.S.A. Dans les usines à simple effet, une solution consiste à placer l'alternateur à l'aval; pour 


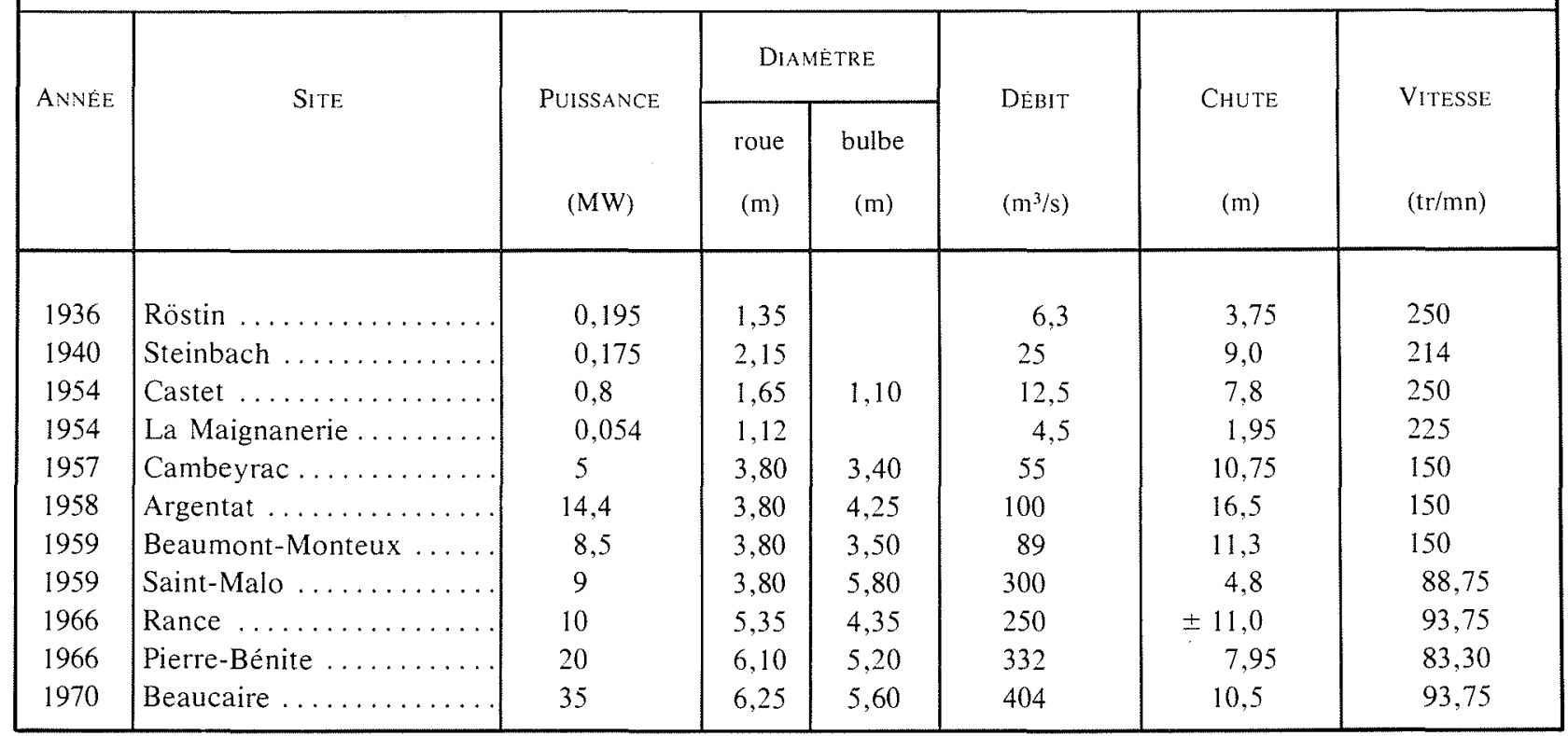

cela il faut réduire le diamètre de l'alternateur et donc améliorer les possibilités de refroidissement, ce qui est difficile pour les grosses machines tournant lentement. Une voie permettant d'intéressants progrès, par l'augmentation de la vitesse des alternateurs, serait l'utilisation de multiplicateurs de vitesse à planétaires inclus dans le bulbe entre la turbine et l'alternateur.

\subsection{Les études de corrosion}

La corrosion des parties métalliques de l'usine dans l'eau de mer fut un des premiers soucis des promoteurs de la Rance. A cet effet fut créé, le 12 juillet 1955, le «groupe corrosion" réunissant les représentants de la S.E.U.M., E.D.F. et G.D.F., avec, comme objectif, l'établissement d'une doctrine sur les dispositions à prendre pour protéger contre la corrosion et les salissures les diverses structures de l'usine de la Rance. Le problème fut attaqué, à la station de Saint-Servan, sous les trois aspects : peintures, métaux nobles, protection cathodique.

\subsubsection{Etude des peintures}

Les essais de plusieurs centaines de revêtements [16a] ont permis d'énoncer comme meilleur choix la peinture vinylique, en épaisseur de l'ordre de $0,15 \mathrm{~mm}$, sous réserve de précautions lors de la mise en œuvre: bon état des surfaces, excellente préparation des fonds, suppression des angles vifs et contrôle de l'épaisseur du film $[27 c]$. Ces impératifs ont conduit E.D.F. à construire un atelier spécial de mise en ouvre de la peinture, laquelle s'avéra être une technique très délicate (maintien du degré nygrométrique pendant l'application, correction des cotes d'usinage pour tenir compte de l'épaisseur du film de peinture, etc.).

\subsubsection{Les alliages}

Le groupe expérimental de Saint-Malo fut aussi un banc d'essai de différents alliages ferreux et cuivreux $[27 e]$. En définitive furent retenues, pour l'exécution des groupes de la Rance, les nuances suivantes dont les compositions nominales sont indiquées entre parenthèses :

- un acier martensitique $17 / 4(17 \%$ de $\mathrm{Cr}, 4 \%$ de $\mathrm{Ni}$ $1 \%$ de Mo) utilisé sous forme de moulée pour les pales, le moyeu de la roue, le distributeur;

- un acier austénitique $(17 \%$ de $\mathrm{Cr}, 10 \%$ de $\mathrm{Ni}$ $2,5 \%$ de Mo) utilisé sous forme laminé pour les manteaux de roue;

- un cupro-aluminium ( $\mathrm{Cu} 81 \%, \mathrm{Al} 9 \%, \mathrm{Fe} 4.5 \%, \mathrm{Ni}$ $4,5 \%, \mathrm{Mn} 1 \%)$ utilisé sous forme de moulée pour les pales.

Finalement 12 pales de l'usine sont en acier $17 / 4$ et 12 en cupro-aluminium.

\subsubsection{La protection cathodique}

La protection cathodique, d'abord expérimentée sur le groupe de Saint-Malo [27d], s'avère nécessaire du fait du voisinage de divers métaux (aciers ordinaires peints et alliages nobles réputés inoxydables). Son rôle est d'annuler la différence de potentiel entre les aciers ordinaires et les métaux plus nobles.

La protection cathodique s'est révélée indispensable pour les aciers ordinaires (la peinture n'assurant pas une protection durable), la visserie et l'acier austénitique. L'expérience de la Rance montre l'intérêt de la protection cathodique; celle-ci s'oppose efficacement à la corrosion de l'acier au carbone, supprime les piqûres sur les aciers inoxydables, elle permet ainsi d'espacer et même de supprimer les reprises de revêtement de pein- 


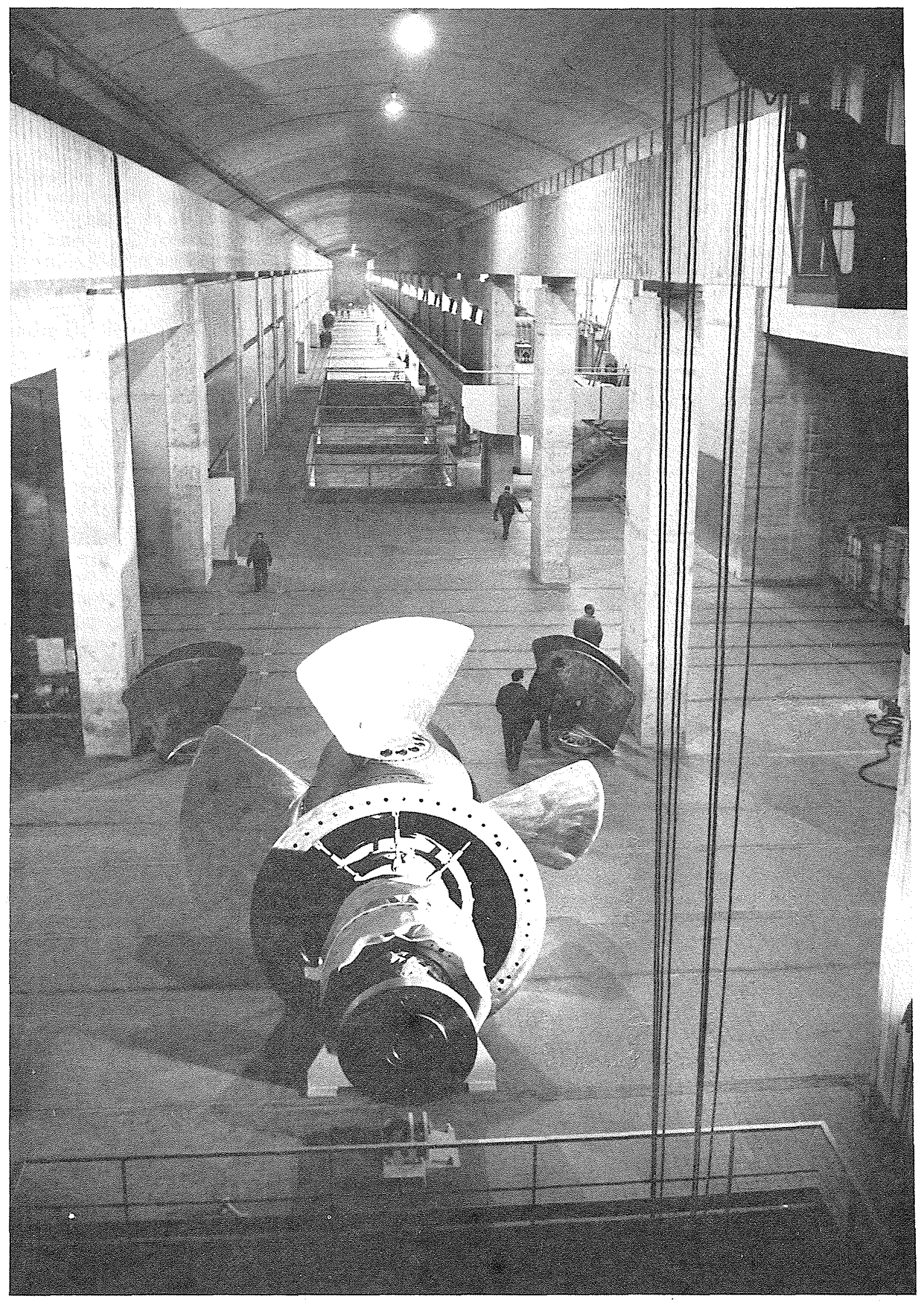


ture. Actuellement, la protection cathodique est étendue aux vannes et à l'écluse; pratiquement, toutes les parties métalliques en eau de mer sont protégées. Dans ces conditions, il serait même possible de se contenter d'un distributeur en acier au carbone [44k, 44l, 44m].

\subsection{L'exploitation de l'usine de la Rance}

\subsubsection{Théorie des cycles d'exploitation}

Le problème original que pose l'usine marémotrice de la Rance est l'élaboration de la doctrine de son exploitation. En effet, il s'agit d'un ouvrage hydroélectrique dont le comportement hydraulique ne s'apparente à aucun type d'ouvrage classique (réservoir, fil de l'eau, pompage); cependant il peut, si on le désire, adopter. selon l'heure, l'un ou l'autre de ces types de fonctionnement.

Les traités sur les cycles d'exploitation des usines marémotrices sont apparus en même temps que les idées d'exploitation des sites [3]. Les premières approches mathématiques de l'élaboration d'une théorie de la recherche du maximum de la production, soit énergétique soit économique, d'une usine marémotrice sont dues à $R$. Gibrat $[4,8]$. Utilisant le calcul des variations et moyennant l'hypothèse simplificatrice de la surface du bassin horizontale, c'est-à-dire ne tenant pas compte des remous amont et aval, il s'agit de rechercher le maximum de la fonction "recette " $U$ entre les deux instants $t_{1}$ et $t_{2}$

$$
U=\int_{t_{1}}^{t_{2}} n N(q, H) p(t) d t
$$

avec:

$n$ nombre de groupes en fonctionnement identiques;

$q$ débit traversant chaque groupe

$H \quad$ hauteur de chute $(H=z-h)$ entre les cotes de l'eau dans la mer $z(t)$ et dans le bassin $h(t)$;

$N(q, H)$ colline des turbomachines (voir exemple fig. 12);

$p(t) \quad$ prix de l'énergie à l'instant $t$;

et sachant, de plus, que l'équipement dispose de vannes utilisables pour obtenir des variations rapides et judicieuses du niveau du bassin.

Parmi les résultats remarquablement simples de cette théorie, est à citer celui selon lequel le point de départ du fonctionnement de l'usine doit satisfaire à la condition de rendement maximal, donc se situer sur la courbe correspondante de la colline $N(q, H)$ (fig. 12). De plus, le point figuratif ne peut franchir la courbe de puissance maximale pour une charge donnée, sinon on consommerait plus d'eau pour une même production. En fait cette règle est enfreinte à la Rance à chaque fin de cycle «double effet» pour réunir les bonnes conditions d'exécution du cycle suivant.

Compte tenu des conditions de limitation de puissance maximale, d'apparition de cavitation, de variation maximale du débit pour ne pas engendrer des intumescences dans l'estuaire préjudiciables aux autres usagers, le problème reste posé $[27 h, 27 i, 35,109 a, 109 b, 109 c]$.

\subsubsection{Le principe de calcul des éléments de la méthode de la Programmation Dynamique}

L'utilisation des calculateurs électroniques a changé le champ d'étude du problème; la recherche du meilleur cycle de fonctionnement est confiée à l'ordinateur en utilisant un processus de recensement logique des différentes possibilités de marche de l'usine. Il s'agit de la “Programmation Dynamique » utilisée par le Centre Interrégional de Mouvements d'Energie de l'Ouest [44a] pour exploiter quotidiennement l'usine de la Rance. Cette méthode, une application de la Recherche Opérationnelle [43], fut d'abord étudiée par SOGREAH; elle fut ensuite appliquée par la Direction des Etudes et Recherches (sur CDC 6600), puis par le Service des Mouvements d'Energie (sur CII 9080) et enfin adaptée par le C.I.M.E.-Ouest (sur CII 9040).

Le principe est de rechercher les couples de points de fonctionnement, chacun défini par deux paramètres (le temps $t$ variant par valeurs discrètes de $10 \mathrm{mn}$, et la cote $y$ de l'eau dans le bassin variant par valeurs discrètes de $10 \mathrm{~cm}$ ) de façon à ce que soit maximale la somme composée :

- d'une part, du gain élémentaire obtenu en passant d'un point à l'autre distant de $10 \mathrm{mn}$ dans le temps, ceci en tenant compte des données extérieures du moment (essentiellement la cote de la mer et le prix de l'énergie) et de toutes les limitations;

- et, d'autre part, de la recette que l'on peut escompter jusqu'à la fin de la période considérée.

Pour ce faire, le calcul comptabilise les gains élémentaires $G\left(y^{\prime}, t^{\prime}, y^{\prime \prime}, t^{\prime \prime}\right)$ entre les instants $t^{\prime}$ et $t^{\prime \prime}=t^{\prime}+10 \mathrm{mn}$ et les variations de cote du bassin $y^{\prime}$ et $y^{\prime \prime}$.

Les éléments du calcul sont les suivants, en valeur moyenne pendant le pas de temps de $600 \mathrm{~s}$ :

- le débit à travers l'usine:

$$
Q T=\frac{S\left(y^{\prime}\right)+S\left(y^{\prime \prime}\right)}{2} \times \frac{\left(y^{\prime}-y^{\prime \prime}\right)}{600}
$$

$S(v)$ représentant la surface du bassin rapportée à la cote $y$ de l'eau mesurée à Saint-Suliac;

- La hauteur de chute:

$$
H=?\left[y_{B}(t)-z\left(t^{\prime}\right)+y_{B}\left(t^{\prime \prime}\right)-z\left(t^{\prime \prime}\right)\right]
$$

$z(t)$ représentant la cote de la mer à l'instant $t$ et $y_{B(t)}$ la cote de la surface de l'eau dans le bassin au droit du barrage; ces deux cotes sont définies, la première à partir de l'annuaire des marées, la deuxième par une relation exprimant le remous du bassin, le seul pris en compte [105, 106] :

- pour le sens d'écoulement mer-bassin :

$$
y_{B}=y+0,13 \cdot 10^{-4} Q^{2}(15-y)^{2}
$$

- pour le sens d'écoulement bassin-mer :

$$
y_{13}=y-0,1.10 \cdots: Q^{2}(15-y)^{*}
$$

(rappelons que y représente la cote de l'eau à Saint-Suliac par rapport au zéro des cartes marines $[105]$ ) ; 
- le débit QV passant par une vanne déduit de:

$$
H=\alpha(Q V / 4)^{*}+\beta(Q V / 4)
$$

expression représentant les caractéristiques des vannes avec

- dans le sens bassin-mer :

$$
\alpha=4,49419 \cdot 10^{-4}, \quad \beta=1,51079 \cdot 10^{-2}
$$

- dans le sens mer-bassin :

$$
\alpha=5,71715.10^{*}, \quad \beta=3,37790 \cdot 10^{-2}
$$

- le débit à travers chaque groupe:

$$
Q G=(Q T-Q V \times N B) / N G
$$

$N B$ et $N G$ représentant le nombre de vannes ouvertes et de groupes en marche entre les instants $t^{\prime}$ et $t^{\prime \prime}$;

- la puissance électrique des groupes:

$$
P=N(Q G, H) \times N G
$$

A ce niveau du calcul, compte tenu du nombre maximal de vannes $N B$ disponibles (elles fonctionnent toutes par tout ou rien), lorsque les groupes doivent fonctionner en pompes $(P<0)$, on essaie de remplacer cette marche par un fonctionnement en "orifice» de puissance nulle, sachant que le débit transitant par un groupe non couplé au réseau est :

$$
Q O=\gamma \sqrt{H}
$$

- avec dans le sens direct (bassin-mer) $\gamma=1820$,

- avec dans le sens inversé (mer-bassin) $\gamma=1420$,

La marche en orifice est choisie si $H>30 \mathrm{~cm}$ et $Q O<Q G$.

Le gain élémentaire pendant le pas de temps de $10 \mathrm{mn}$ ( $1 / 6$ d'heure) est alors calculé à partir de $P$ exprimé en $M W$, par :

$$
G\left(y^{\prime}, t^{\prime}, y^{\prime \prime}, t^{\prime \prime}\right)=(P / 6) p(t)
$$

avec $p(t)$ coût de l'énergie en F/MWh, fourni par le Service des Mouvements d'Energie, correspondant, pour la semaine $S$, aux coûts des jours de la semaine $S-2$, car il est nécessaire de préparer le programme de marche suffisamment à l'avance, puisque le programme prévisionnel de marche doit être diffusé auprès des usagers de l'estuaire au moins $48 \mathrm{~h}$ à l'avance. Ces prix se présentent hebdomadairement sous forme de 4 séries de 24 valeurs ( 1 par heure de la journée) : une série pour le samedi, une pour les dimanches et jours fériés, une pour le lundi ou le lendemain d'un jour férié et la quatrième pour les autres jours ouvrables. Ils sont transposés à la semaine $S+2$ en tenant compte des éventuels jours fériés et leur lendemain. Ils représentent le coût de l'énergie à l'époque considérée s’il fallait la produire par une centrale thermique. Ils sont appliqués à l'énergie produite ou consommée aux bornes de la Rance sans tenir compte des pertes en ligne. Cette suite de prix horaire est découpée en une suite de prix de 10 en $10 \mathrm{mn}$ après lissage, de façon à éviter les variations brusques conduisant à des fonctionnements intempestifs de l'usine.

\subsubsection{Les limitations}

Les limitations «prévisibles» sont de deux sortes:

\subsubsection{Limitations CONTRACTUELles}

La concession de l'usine marémotrice de la Rance impose les contraintes suivantes [47] à l'exploitation de l'usine :

- Les cotes de l'eau dans le bassin ne doivent pas descendre au-dessous du zéro des cartes marines, ni atteindre des valeurs telles que soient submergés les terrains situés au-delà cles limites fixées par le décret du 10 janvier 1962 et déterminées par le plus grand flot du 17 mars 1957.

- Le niveau ne doit pas dépasser :

- la cote +13 plus de $8 \mathrm{~h}$ consécutives ni plus de 40 fois par an et $250 \mathrm{~h}$ au maximum;

- la cote $+12,5$ plus de 16 h consécutives ni plus de 300 fois par an et $900 \mathrm{~h}$ au maximum (fig. 13).

- Le niveau, à Saint-Suliac, ne doit pas varier de plus de $4 \mathrm{~m}$ en $1 \mathrm{~h}$, ni plus de $1 \mathrm{~m}$ en $10 \mathrm{mn}$ (exceptionnellement $1,4 \mathrm{~m}$ en cas d'incident).

De plus, d'après le cahier des charges, E.D.F. doit communiquer chaque jour, avant $6 \mathrm{~h}$, le programme de marche du lendemain jusqu'à 24. h. De plus, E.D.F. doit fournir, avant le 31 mars, les horaires, entre le 15 juin et le 15 septembre, pendant lesquels le niveau à SaintSuliac sera supérieur ou égal à $+4 \mathrm{~m}$ entre 7 et $21 \mathrm{~h}$, et à $+8,5 \mathrm{~m}$ entre 8 et $20 \mathrm{~h}$.

\subsubsection{LIMITATIONS TECHNOLOGIQUES}

En dehors de l'aspect économique, pour des raisons d'exploitation, le pompage est soit limité, soit interdit, pendant certaines heures de la journée, pour éviter des surcharges sur les lignes du réseau d'interconnexion, ou en cas de retraits d'exploitation de certains ouvrages.

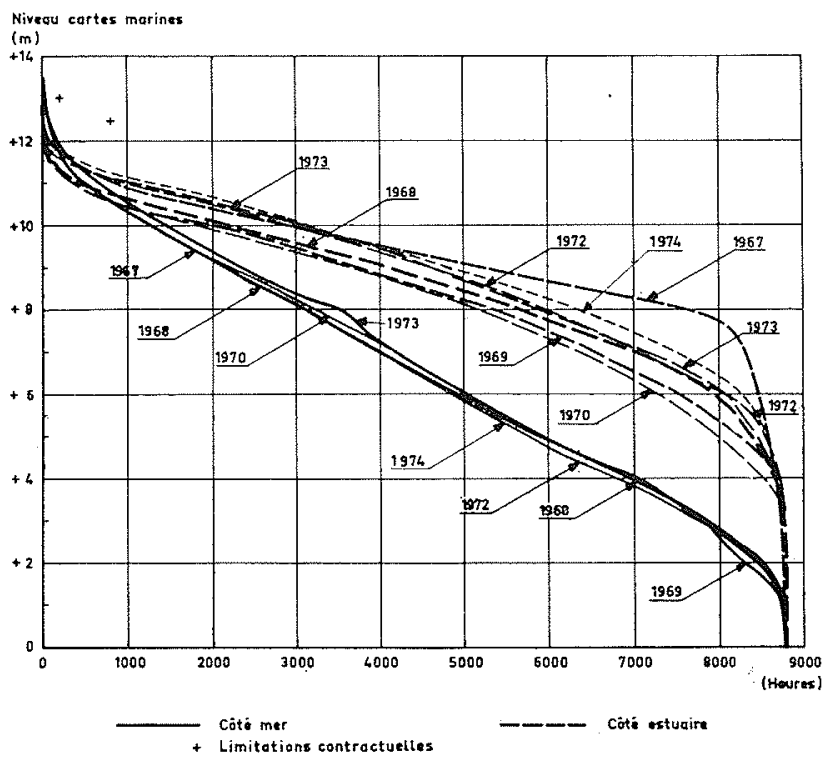

13/ Niveaux classés de part et d'autre du barrage de la Rance 


\subsubsection{Recherches du fonctionnement optimal}

Les gains $G\left(y^{\prime}, t^{\prime}, y^{\prime \prime}, t^{\prime \prime}\right)$ étant connus, la recherche $\mathrm{du}$ fonctionnement optimal consiste à déterminer la suite des $y^{\prime}, y^{\prime \prime}$, etc., optimale pendant une période fixée (la semaine du samedi $0 \mathrm{~h}$ au vendredi $24 \mathrm{~h}$ ) de durée $T$. Dans une première phase le calcul remonte le temps; partant de $t^{\prime}=T-10 \mathrm{mn}$, pour tout $y^{\prime}$ de la gamme de variation possible à cette époque, il détermine le $y^{\prime \prime}$ optimal correspondant à $t=T$, et ainsi de suite à partir de $t^{\prime}-20 \mathrm{mn}$, etc., jusqu'à $t=0$, en donnant de plus la recette correspondante, somme des gains optimaux depuis $t=T$. Les chemins optimaux ne sont pas forcément ceux qui aboutissent aux cotes discrètes distantes de $10 \mathrm{mn}$; ils sont interpolés entre ces derniers.

Dans la deuxième phase, connaissant la cote $y_{0} \mathrm{du}$ bassin à l'instant initial, le calcul a pour but de déterminer la loi de variation de la cote du bassin conduisant à la recette maximale. Le premier chemin élémentaire, à partir des points $y=y_{0}, t=0$, s'obtient par interpolation entre les chemins élémentaires optimaux encadrant $y_{0}$. A chaque point de cote $y_{1}$ à l'instant suivant $(t=10 \mathrm{mn})$ correspond un gain entre $y_{0}$ et $y_{1}$ et une recette due au fonctionnement de l'usine jusqu'à $t=T$, recette qui peut etre obtenue par interpolation entre les deux recettes correspondant aux deux cotes encadrant $y_{1}$, pour lesquelles elles sont connues par les calculs de la première phase. La cote $y_{1}$ choisie est celle qui maximise la somme des recettes de $t=10 \mathrm{mn}$ à $t=T$ et du gain de $y=y_{0}, t=0$, à $y=y_{1}, t=10 \mathrm{mn}$. Le calcul reprend alors en partant du $y_{1}$ optimal pour déterminer $y_{2}$ cote optimale du bassin à l'instant $t=20 \mathrm{mn}$.

Le calcul peut conduire à des décisions optimales, demandant des ouvertures de vannes ou de mises en service de groupes intempestives; celles-ci ne sont adoptées que si les étapes de fonctionnement optimales (turbinage, pompage, vannage, etc.) sont supérieures à $30 \mathrm{mn}$. Pour respecter cette limitation, à chaque point $y$, $t$ sont associés en plus de la recette entre $t$ et $T$, les modes de fonctionnement en cours et le suivant, c'est-à-dire après changement de sens de l'écoulement. De même, pour réduire le nombre de démarrages électriques, contraignant pour le matériel, est supprimé tout démarrage en pompe devant intervenir plus d'une heure après l'instant d'égalité des niveaux bassin et mer.

\subsubsection{Prise en compte des aléas}

Entre la date de sortie du programme et sa réalisation des événements peuvent survenir. Si les aléas interviennent plus de 3 jours avant la réalisation du programme, celui-ci est modifié; le programme rectifié est diffusé aux usagers. En cas d'avaries d'ouvrages intervenant à moins de $48 \mathrm{~h}$ de la réalisation du programme, le dispatching adopte un nouveau schéma en diminuant en général les pompages; les usagers sont prévenus dans les meilleurs délais.

Les aléas survenant en cours de réalisation sont de deux types: avaries de matériel (réseaux ou groupes), ou aléas dus à la marée. Ces derniers constituent des écarts de phase (maximum de $20 \mathrm{mn}$ ) ou d'amplitude par rapport aux valeurs prévues et utilisées pour l'établissement du programme. Lorsque les écarts sont fai- bles, le calculateur de l'usine procède à un « microrattrapage » qui consiste à modifier l'orientation des pales des groupes (dans les limites de $\pm 4^{\circ}$ ) par rapport au programme prévu, en changeant les débits de façon à ce que le niveau prévu dans le bassin soit "rattrapé » dans un délai de $2 \mathrm{~h}$, les ordres de correction étant donnés toutes les $10 \mathrm{mn}$. Cette méthode de correction est efficace en cas d'écart de l'amplitude; l'écart ne changeant pas de sens au cours de la marée, la correction peut s'effectuer progressivement. En revanche, la méthode de correction conduit à des ordres absurdes en cas d'écart de phase; par exemple, à basse mer, l'écart change de sens et les ordres de correction ont alors pour objet de l'accroître. Dans ces cas, ainsi que dans le cas d'aléas sur le réseau, l'usine est commandée manuellement par le chef de quart, ce qui permet de parer au dirigisme du microrattrapage grâce à une vision intelligente de l'avenir. Mal conduit, le microrattrapage entraînerait une perte d'énergie qui pourrait être de l'ordre de quelques pour cent et que la correction manuelle permet de récupérer.

\subsection{Les performances de la Rance}

L'usine fonctionne en totalité depuis 1968; le tableau 3 (performances) montre deux faits [108] :

- d'une part, la disponibilité des groupes augmente, ce qui est à mettre à l'actif de la gestion de l'entretien de l'usine, puisque l'indisponibilité comprend la totalité du temps nécessaire à l'entretien, lequel s'effectue normalement en dehors des périodes de production d'énergie:

- d'autre part, la production énergétique annuelle croît; en 1974, avec $507 \mathrm{GWh}$, elle a atteint $93 \%$ de la production théorique de $544 \mathrm{GWh}$; ceci est dû surtout à la réduction des limitations de pompage due à l'amélioration du réseau d'interconnexion. Mais, en fait, le principe de la gestion étant d'obtenir la recette maximale, le critère de production énergétique annuelle n'a qu'un sens indicatif (').

Des comparaisons des fonctionnements simulés de la Rance, obéissant aux critères de production ou de recette maximales, font apparaitre très peu de différence en vive-eau; en revanche, en morte-eau, le critère du maximum d'énergie accrô̂t la production de $30 \%$ par rapport au critère du maximum de recette. Ceci veut-il dire que la Rance est une usine de base en vive-eau, et une usine de pointe en morte-eau? En fait, en vive-eau, il ne serait pas impossible d'obtenir de l'énergie de pointe, grâce à une modulation plus importante des prix de l'énergie, en acceptant de ne pas produire de l'énergie mal placée. En revanche, en morte-eau, le bassin peut être utilisé comme un réservoir.

Certaines rubriques du tableau des performances de la Rance méritent quelques commentaires. La production brute est la production des groupes diminuée de la consommation des auxiliaires (ce ne serait pas la pro-

(1) A partir de 1975, et pendant plusieurs années, la production de l'usine de la Rance sera malheureusement réduite de $20 \%$ environ par suite de l'arrêt pour réparation de tous les groupes successivement et la suppression, jusqu'à l'achèvement de la réparation, des marches en turbine inversée et en pompage direct qui paraissent avoir été responsables des dommages constatés sur les alternateurs. 


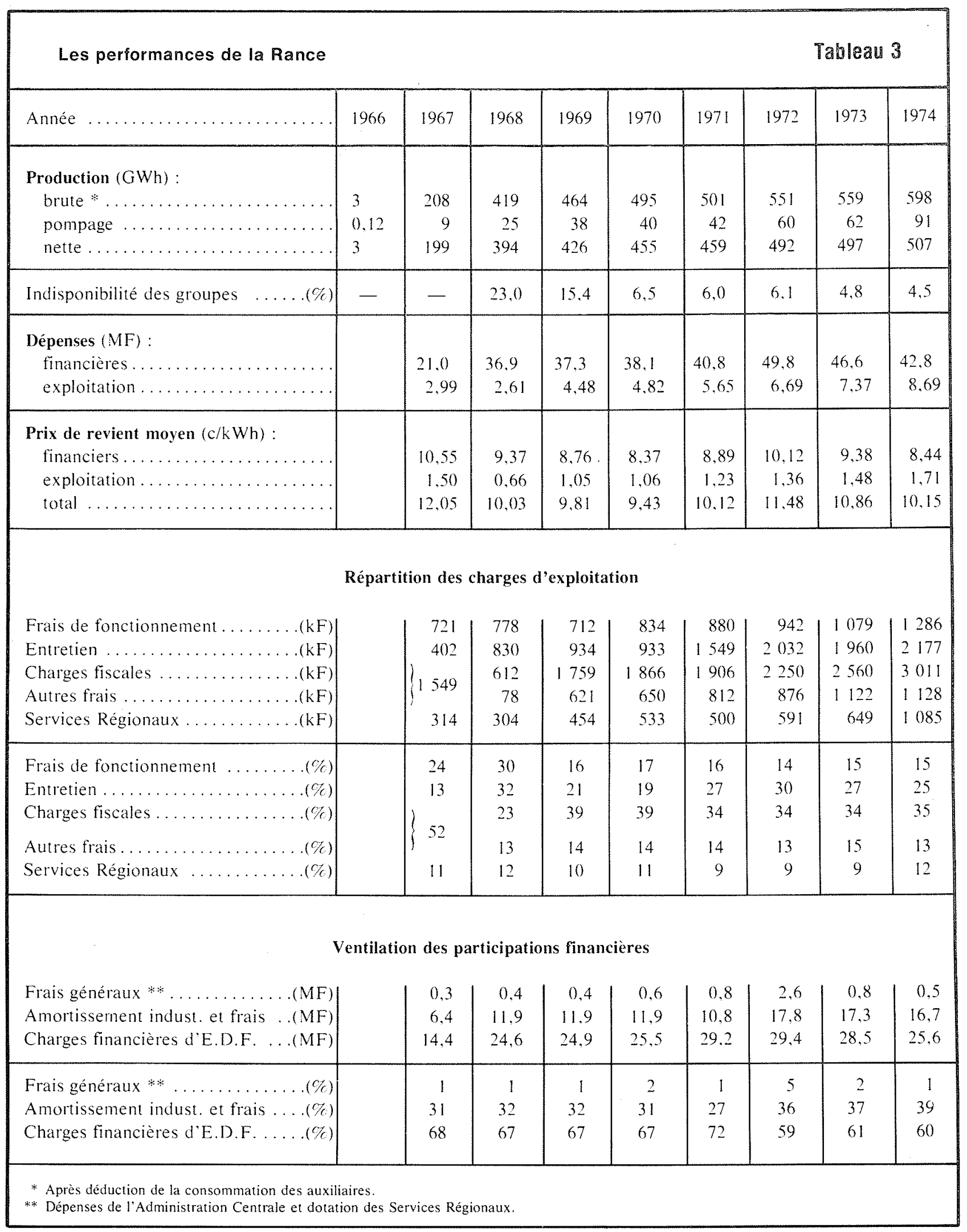


duction de l'usine sans pompage). Les prix de revient du kWh sont calculés en divisant les dépenses (qui ne tiennent pas compte de l'achat d'énergie de pompage) par la production nette, c'est-à-dire celles effectivement apportée au réseau; il s'agit d'un prix de revient moyen ne tenant pas compte des variations du prix de l'énergie, et qui a un sens dans la mesurz où le prix de l'énergie de pompage est le même que celle produite. Pour essayer de préciser ce point, nous avons estimé les dépenses de pompage et calculé ensuite le prix de revient en divisant les dépenses totales par la production brute.

Il est intéressant de noter que les différents composants des frais d'exploitation ont sensiblement la même importance chaque année; en moyenne ils se répartissent de la façon suivante :

- frais de fonctionnement ......... $18 \%$

- frais d'entretien ............... $26 \%$

- charges fiscales ............... $34 \%$

- autres frais ................. $12 \%$

— frais directs des services régionaux ... $10 \%$

Dans le tableau 3, l'incidence des frais d'exploitation sur le $\mathrm{kWh}$ produit est calculée en francs courants; or, calculé en francs constants, ce prix augmente avec le temps: comme la répartition des différentes rubriques de ces frais est constante, il n'est pas possible d'imputer celte dérive à une dépense plutôt qu'à une autre.

\begin{tabular}{|c|c|c|c|c|c|c|}
\hline \multicolumn{6}{|c|}{$\begin{array}{l}\text { Incidence des frais d'exploitation } \\
\text { sur la production (c 1974/k Wh) }\end{array}$} \\
\hline $1967 / 68$ & 1969 & 1970 & 1971 & 1972 & 1973 & 1974 \\
\hline 1,34 & 1,37 & 1,32 & 1,45 & 1.52 & 1,57 & 1,71 \\
\hline
\end{tabular}

De même la ventilation des charges financières est assez constante d'une année à l'autre :

- frais généraux .............. $1 \%$

- amortissement de l'installation...... $33 \%$

- charges financières d'E.D.F. ....... $66 \%$

Il convient de préciser que la rubrique dépenses d'amortissement résulte de l'amortissement du capital du génie civil sur 60 ans et du matériel électromécanique sur 30 ans, plus les frais d'établissement de l'année en cours; les intérêts de ces sommes ne sont pas pris en compte. En revanche, les charges financières des emprunts E.D.F, en cours sont réparties sur l'ensemble des usines (au prorata du montant non amorti des investissements non réévalués); elles constituent la plus lourde part du prix de revient (environ $60 \%$ ).

Avec cette méthode de calcul le prix de revient apparent $\mathrm{du} \mathrm{kWh}$ de la Rance revient à environ $11 \mathrm{c}$. II faut préciser que, dans ce calcul, le prix de l'énergie achetée pour le pompage est supposé égal au prix de vente de l'énergie à l'usine.

La part des charges financières étant la plus impor- tante, il est intéressant de distinguer, dans le prix de revient du $\mathrm{kWh}$, la part due à l'amortissement et aux charges d'exploitation, et celle due aux charges financières.

Ceci donne en francs constants 1974 (cf. tableau 5).

\section{Prix de revient moyen}

(c 1974/kWh)

Tableau 5

\begin{tabular}{|c|c|c|}
\hline AvveEs & $\begin{array}{c}\text { Charges PRopres } \\
\text { A L.USINE }\end{array}$ & $\begin{array}{c}\text { Charges FIVA VCIERES } \\
\text { D.E.D.F. }\end{array}$ \\
\hline 1967 & 6,78 & 10,50 \\
1968 & 5,28 & 9,06 \\
1969 & 5,02 & 7,62 \\
1970 & 4,74 & 6,94 \\
1971 & 4,44 & 7,52 \\
1972 & 6,16 & 6,68 \\
1973 & 5,44 & 6,07 \\
1974 & 5,10 & 5,05 \\
\hline
\end{tabular}

Ce qui donne en moyenne pour les six dernières années:

- $5,15 \mathrm{c} / \mathrm{kWh}$ du fait de l'amortissement et des charges d'exploitation;

- 6,65 c/kWh du fait des charges financières d'E.D.F.

Le $k W h$ de la Rance apparait ainsi à un niveau de prix voisin de celui auquel s'est élevé le $\mathrm{kWh}$ thermique classique à la suite de la crise pétrolière. Comme pour ce dernier, la comparaison avec le $\mathrm{kWh}$ nucléaire est très défavorable.

V.B. - Le calcul qui préciede est un simple calcul rétrospectif at comptable. Il est sans rapport anece an calcul de rentabilité économiane al'ant décision.

\subsection{Analyse du fonctionnement de l'année $\mathbf{1 9 7 3}$}

L'analyse du fonctionnement de la Rance pendant l'année 1973 a été faite à partir des données élaborées par la Programmation Dynamique; elle concerne donc un fonctionnement théorique, non corrigé de l'influence des aléas dus à la marée, en quelque sorte un fonctionnement de l'usine idéale, tenant cependant compte des aléas liés aux indisponibilités des groupes et des vannes.

La figure 14 présente la répartition de l'énergie et de la puissance en fonction de l'heure de la journée. Elle montre que la Rance marche à "pleine puissance", donc produit de l'énergie, de 0 à $5 \mathrm{~h}$ du matin (heures creuses) et entre 12 et $17 \mathrm{~h}$ (heures pleines); plus précisément, de 1967 à 1974 inclus, $54 \%$ de la production de la Rance se place en heures de pointe et en heures pleines (heures qui représentent $57 \%$ des 8760 heures de l'année). 


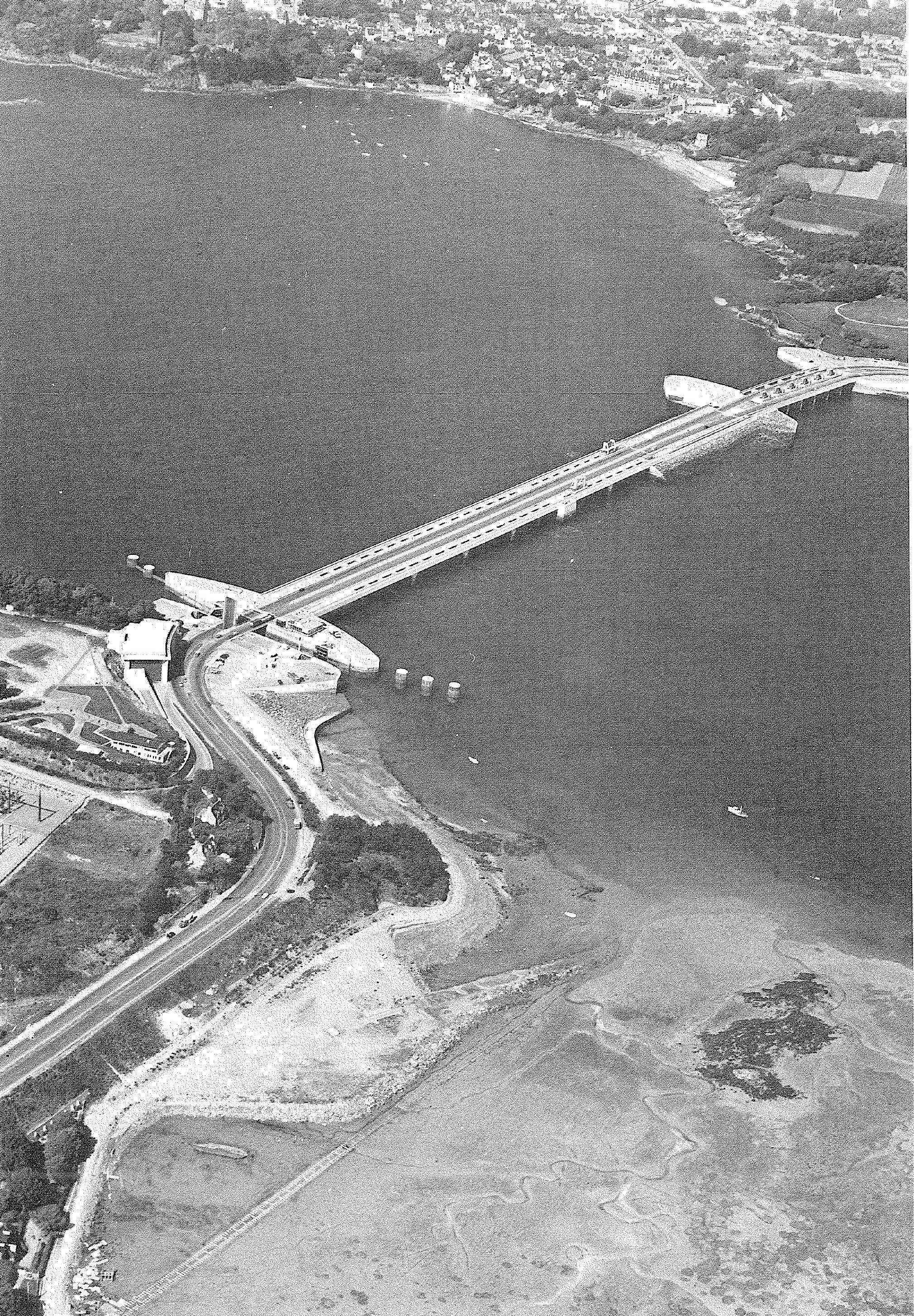


Quelles sont les causes de ce type de fonctionnement? Deux hypothèses se présentent :

- Les pleines-mers de vive-eau ont lieu à Saint-Malo vers 7 et $19 \mathrm{~h}$; à cette époque la chute est faible; en revanche, les fortes chutes ont lieu 3 heures avant. Ceci suffit pour expliquer le diagramme énergétique obtenu. Il serait cependant possible d'obtenir des maximums énergétiques aux heures de pointe si le fonctionnement en turbinage inversé avait un meilleur rendement.

- Aux heures de pointe nationales, la Rance «pompe »; cette situation est due au faible écart entre les prix de l'énergie produite et l'énergie pompée (fig. 15). Il en résulte que le critère de fonctionnement de l'usine est très proche de celui conduisant à la production maximale d'énergie; or les pleines-mers de vive-eau, c'est-à-dire les époques où il est intéressant de pomper, se situent aux heures de pointe. Cependant il n'est pas du tout paradoxal d'envisager un pompage aux heures pleines ou de pointe si les puissances disponibles sur le réseau le permettent et tant que le rapport entre les coûts de l'énergie est inférieur au rendement du pompage de la Rance, soit $280 \%$.

En fait, l'usine étant sous-équipée en vive-eau, les heures de production se situent forcément à mi-marée et la modulation du prix de l'énergie au cours de la journée n'a pas d'influence notable sur le fonctionnement. En revanche en morte-eau, l'usine sur-équipée a des capacités de pompage qui lui permettent de concentrer sa production à certaines périodes de la journée. selon la valeur de l'énergie; le fonctionnement s'apparente alors à celui d'une usine de pointe.

La figure 14 montre que la production est bien répartie dans l'année. La figure 15 met en relief la répartition annuelle suivante des différents types de fonctionnement (cf. tableau 6).

\begin{tabular}{|c|c|c|c|c|}
\hline \multicolumn{5}{|c|}{ Tableau 6} \\
\hline TYPE DE FONCTIONNEMENT & $\begin{array}{l}\text { DUR } \\
\text { (h) }\end{array}$ & $\begin{array}{l}\mathrm{REE} \\
(\%)\end{array}$ & $\begin{array}{l}\text { Pronue } \\
(G W h)\end{array}$ & (\%) \\
\hline En pompage .......... & 1300 & 15 & -50 & 8 \\
\hline Puissance réduite $(0-50 \mathrm{MW})$ & 4000 & 45 & 30 & 5 \\
\hline Mi-puissance $(50-150 \mathrm{MW}) \ldots$ & 1500 & 17 & 150 & 24 \\
\hline Pleine puissance $\ldots \ldots \ldots$. & 2000 & 23 & 400 & 63 \\
\hline
\end{tabular}

En définitive, en 1973, la Rance a fonctionné à pleine puissance (150-250 MW) pendant 2000 heures, la plupart entre 22 et 5 h et 10 et 16 h. Les 4000 heures de marche à puissance réduite correspondent, pour la plupart, aux périodes d'attente; les unes, normales, sont inhérentes au principe même de fonctionnement d'une usine marémotrice (fig. 2 à 4 ); les autres sont dues aux contraintes limitatives, en particulier les interdictions de pompages par suite des risques de surcharge du réseau

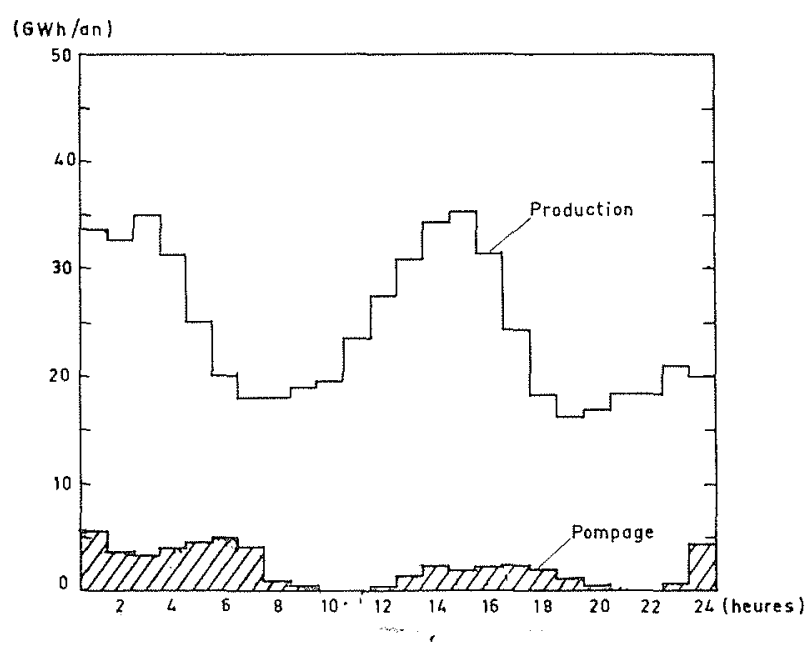

14 a/ Répartitlon journallère de l'énergle tołale annuelle

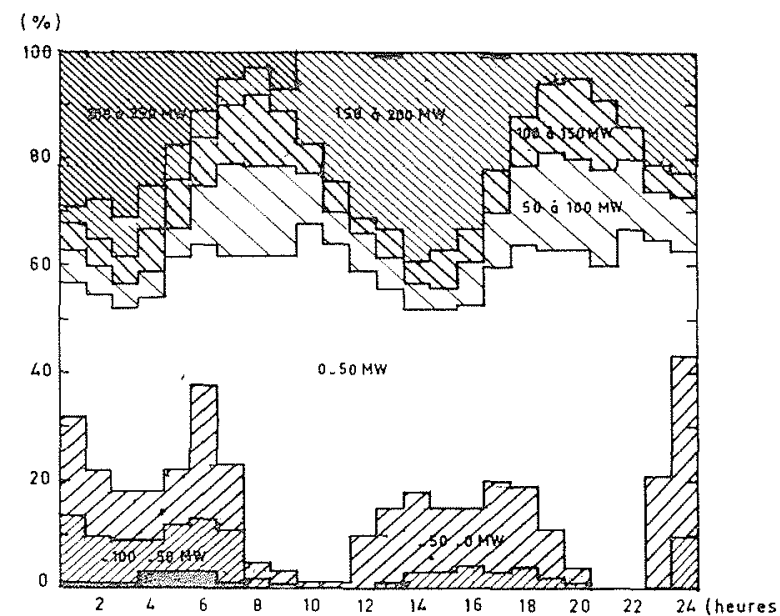

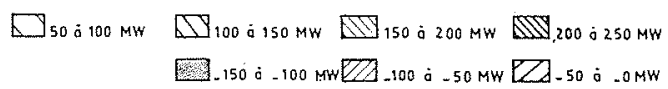

14 b/ Répartition journallère de la puissance moyenne horalre

de transport $\left({ }^{2}\right)$, et aussi à la suppression des fonctionnements de courte durée que peut proposer la programmation dynamique. On voit, sur ce dernier exemple, combien les ordres du programme aveugle gagnent à être intelligemment corrigés.

Le critère d'exploitation de la Rance en énergie maximale conduirait à pomper à pleine-mer de vive-eau, c'est-à-dire à l'heure de pointe. Le critère d'exploitation en recette maximale n'empêche pas cette situation paradoxale, par suite de la faible différence des prix affectés à l'énergie produite et à l'énergie consommée. Cette remarque remet en cause le principe de la recette

(2) En particulier à $22 \mathrm{~h}$, départ du tarif "heures creuses " donc du démarrage des chauffe-eau électriques, ce qui se traduit par une pointe de trafic très sensible en Bretagne où la charge industrielle est faible. La figure 14 montre dailleurs que la Rance " ne pompe pas " à cette heure de pointe régionale 

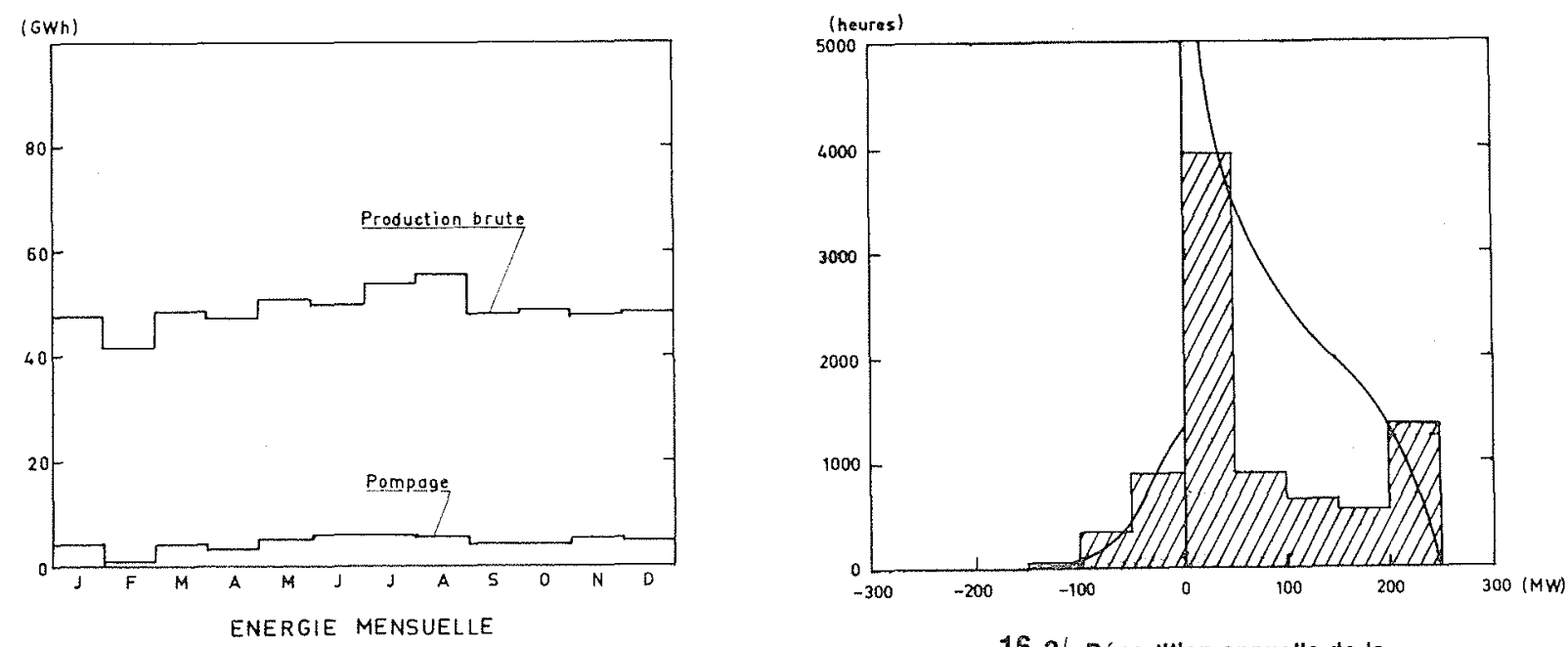

16 a/ Répartition annuelle de la pulssance moyenne

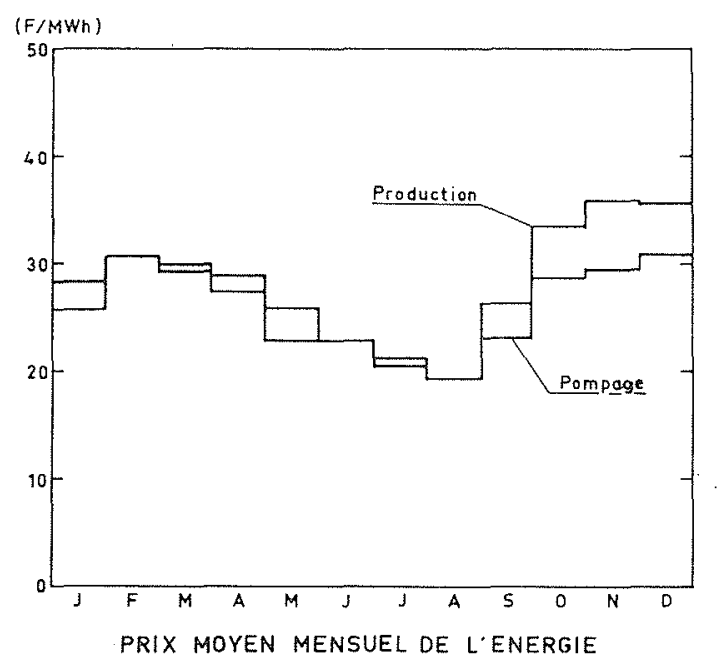

15/ Répartition de la production et du prix de l'énergie en 1973 à la Rance maximale qui a évidemment pour effet de réduire la production, donc joue au détriment de la Rance; laquelle, comme pour toutes les autres usines, est "jugée" sur sa production annuelle. On peut donc se demander s'il ne serait pas plus simple d'exploiter la Rance suivant le critère du maximum d'énergie, quitte à introduire des interdictions de pompage aux heures de pointe, le gain en simplicité se situant au niveau des calculs des cycles. Toutefois, la continuation de l'utilisation d'un ordinateur permettrait de mieux apprécier la gestion de l'usine si les trois hypothèses (recette maximale, énergie maximale avec ou sans limitation de pompage) étaient examinées conjointement et le choix définitif effectué à partir de leur comparaison.

Le Centre Interrégional des Mouvements d'Energie de l'Ouest a effectué des études de l'influence de quelques paramètres en simulant le fonctionnement de l'usine après modification des contraintes; il apparaît. qu'en donnant plus de possibilités au pompage, on améliore les bilans économiques et énergétiques, sauf en morte-eau où on pénalise le bilan énergétique. Un résultat intéressant à connaître est que l'indisponibilité d'une vanne (sur 6) diminue la production de 2 à $3 \%$.

La figure 13 montre que le fonctionnement de l'année 1973 est semblable à celui des années précédentes, sauf l'année préparatoire 1967. En 1974, la réduction des limitations de pompage a eu pour effet une augmentation de la production de $10 \mathrm{GWh}$; comment est répartie cette énergie est une question qui mérite l'analyse.

La figure 13 traduit enfin une des formes des effets de l'usine sur l'environnement: la conclusion est que, d'une part, les niveaux moyens du bassin semblent globalement relevés de $3 \mathrm{~m}$; d'autre part, les niveaux supérieurs, autrefois dommageables pour les riverains, sont aujourd'hui inférieurs de 0,5 à $1 \mathrm{~m}$ à ceux qu'imposerait la marée naturelle. 


\subsection{Conclusions sur la Rance}

En fait, le succès de la réalisation de la Rance n'a rien d'original; la stratégie est basée sur la division du travail en cinq volets classiques:

- calculs théoriques;

- bureaux d'études;

- modèles physiques;

- mesures en nature;

- expérimentation des matériels;

avec l'évident, mais fondamental, gage de succès : une coordination acceptée par tous qui a su créer un esprit de coopération jamais troublé par quelque velléité de compétition ou d'interférence entre les différents participants. Peut être aussi parce que l'ouvre ne rencontra pas, ou peu, d'opposition politique, philosophique ou humaine, et que les seuls obstacles étaient technologiques et économiques.

\section{Le projet des îles Chausey (1)}

\begin{abstract}
"Tous $\left({ }^{2}\right)$ concourent à amortir le choc de cette onde monstrueuse que les influences combinées de la lune et du soleil évoquent deux fois le jour de limmensurable étendue, et qui sent doubler sa puissance quand elle vient s'angustier dans l'étroit entonnoir de la Manche. "
\end{abstract}

A. DE CHEVREMONT [2]

\begin{abstract}
"Ce que le vent a détruit, le vent doit le reconstruire » dit le proverbe. Selon la légende, au printemps 709, une grande marée d'équinoxe violemment poussée par les vents détruisit l'immense forêt de Scissey; puis, progressivement selon les uns, brutalement selon les autres, la mer en vint à buter sur les rochers de SaintMalo, de Dol et de Granville, tandis que le flot élargissait la Rance (fig. 17). Depuis, dès 1024, sous l'impulsion d'Alain V, duc de Bretagne, les hommes, utilisant la force des vents, ont repris à la mer le marais de Dol; conquête suivie d'autres succès, puis d'échecs comme en témoigne la digue de la Roche-Torin inachevée il y a un siècle.
\end{abstract}

A chaque siècle sa conquête: au début $\mathrm{du} \mathrm{XX}^{\mathrm{e}}$ siècle, l'effort de poldérisation ralentit; la course à l'énergie commence. Est-ce le souvenir de l'ancien cordon littoral barrant la baie du Mont Saint-Michel, de Cancale à Carolles, qui inspira dès 1942 le premier projet d'usine marémotrice dans la baie? Puis, pendant vingt ans le projet s'affine; les ingénieurs prennent conscience des difficultés réelles de l'entreprise. Mais l'enjeu est tentant : 10 à $12 \mathrm{GW}, 20$ à $30 \mathrm{TWh} / \mathrm{an}$.

Avec le projet d'usine marémotrice des iles Chausey. souvent appelée aussi "du Mont-Saint-Michel », le rêve

(1) La plus ancienne mention se trouve dans Venämius Fortunatus: (VI' siècle) : Scessiacum, d'où Scessiac, Scessi, Chezy, Chezey (y final égalant ey en vieux français et en anglais). Chosey dans les portulans du XVe et XVII ${ }^{e}$, enfin Chausey.

(2) Les archipels des Minquiers et de Chausey. des ingénieurs devient un défi à relever. Les difficultés sont à l'échelle de l'entreprise : d'abord construire 30 à $50 \mathrm{~km}$ de digues en pleine mer, dans une zone où les courants de marée sont violents et les tempêtes très dures, sur un sol qui se dérobe car le bon rocher est loin; ensuite orchestrer le fonctionnement d'un millier de machines d'une puissance totale de l'ordre de grandeur de la moitié de la puissance installée en France en 1960. Ce combat technique, les ingénieurs se sont préparés à l'affronter pendant environ quinze ans (19501965). Cette provocation se double d'un défi moral et philosophique : aura-t-on le courage d'engager l'entreprise - 10 à 20 ans de travail - alors que les règles de gestion prudente utilisent des outils économiques dont la portée n'est que de quelques années; alors comment se persuader, puis convaincre de l'effort financier à faire.

Cette période d'étude préparatoire est décrite dans ce chapitre, tour d'horizon de ce qui a été fait, rappel des idées émises, des hésitations des ingénieurs, plate-forme de départ.

\subsection{Les études océanographiques}

\subsubsection{La marée}

\subsubsection{LES MESURES DE COURANT}

En vue de l'établissement d'un avant-projet de l'usine marémotrice, le S.E.U.M. lança en 1953 [206] une première campagne de mesures en nature de marée afin de compléter les renseignements publiés par le Service Hydrographique de la Marine; il s'agissait de mesures de courant à l'aide de courantographes (E.D.F. .et B.B.T.-Neyrpic) à 2,5 et $5,5 \mathrm{~m}$ au-dessus du fond, et par la méthode du loch à $5 \mathrm{~m}$ de la surface.

Les opérations ont duré du 13 mai au 24 octobre 1953, et consisté en 33 stations de mouillages au cours desquelles furent enregistrées 240 marées. Il en a résulté le tracé des cartes de courants pour les coefficients de marée 45, 72 et 100 [207] dont la figure 18 donne un exemple, tout en tenant compte des quelques mesures complémentaires qui ont eu lieu en 1954 dans la région pointe du Grouin-phare du Herpin.

Par la suite, le Laboratoire National d'Hydraulique, chargé de construire le modèle réduit du golfe de Saint-Malo en vue des études préliminaires de l'usine des îles Chausey, demanda au S.E.U.M. d'entreprendre une campagne de mesures de courants couvrant l'étendue représentée sur le modèle réduit. Cette mission, effectuée au cours des années 1957, 1958 et 1959 , fournit un très grand nombre d'enregistrements $(81$ points de mesures où les durées vont de 200 à 1500 heures). Les courantographes utilisés étaient de types Dunkerque et Chausey, d'une autonomie de 15 jours, mis au point par le Département des Essais Extérieurs de la Direction des Etudes et Recherches; ils étaient immergés à 5 ou $10 \mathrm{~m}$ du fond. Quelques mesures de répartition de vitesse sur la verticale, à l'aide de moulinets, ont montré que les vitesses au fond étaient inférieures d'environ $20 \%$ de celles de la surface. Les mesures ont servi à définir les courbes de courants types pour les coefficients de marée 45,70 et 95 et à dresser les cartes de courants du golfe de Saint-Malo pour les 


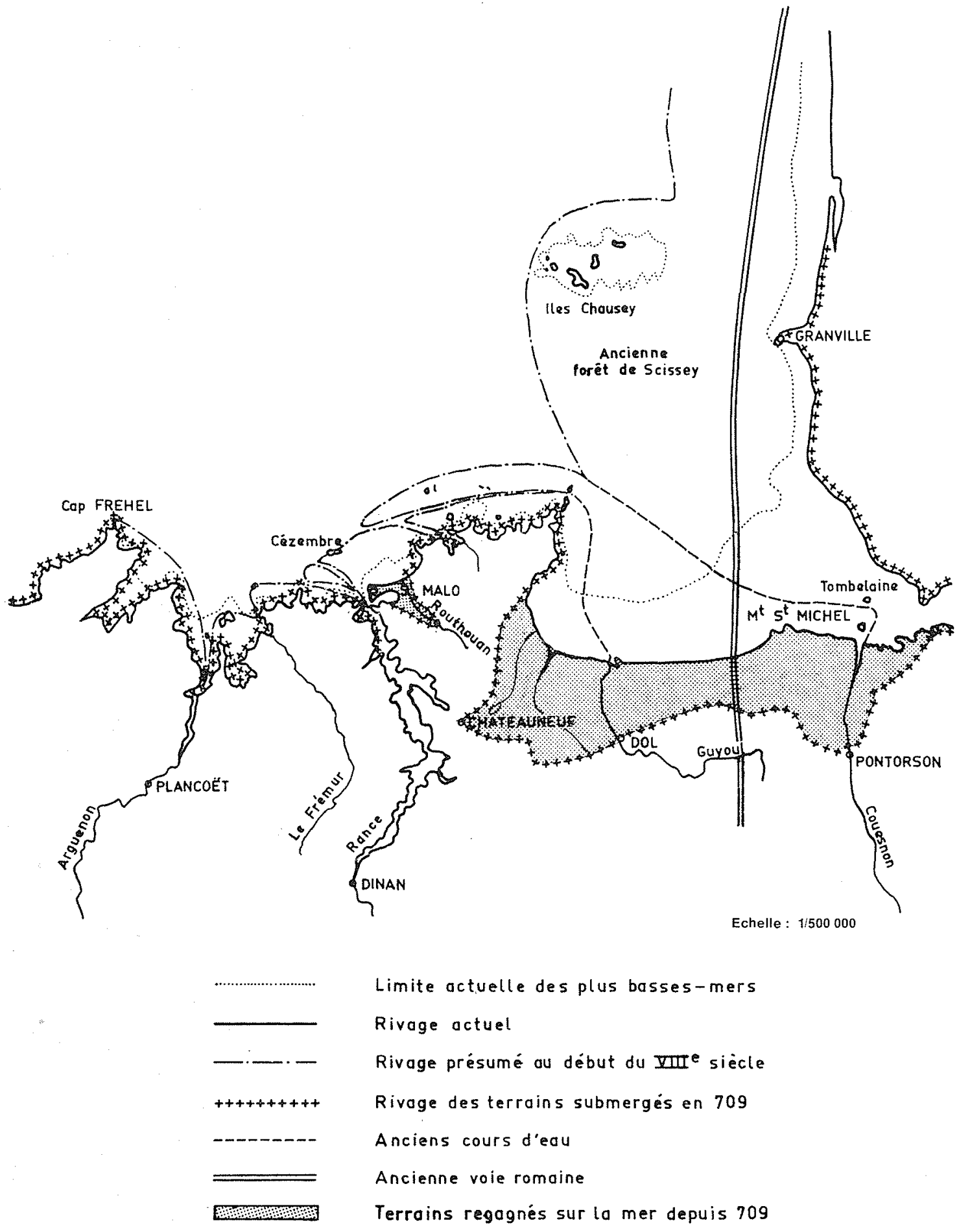

17/ Les mouvements du rivage dans la baie du Mont-Saint-Michel 


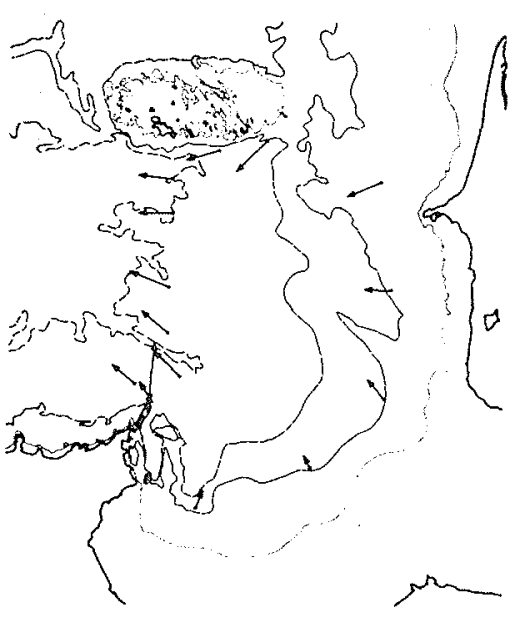

GH AVANT PM A SAINT SERVAN

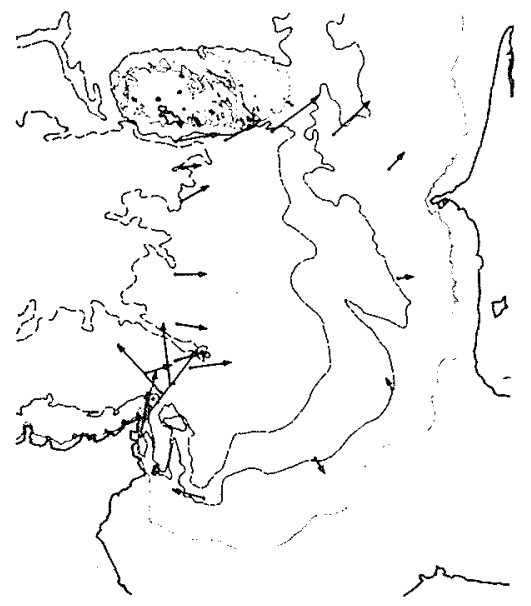

A PM A SAINT SERVAN

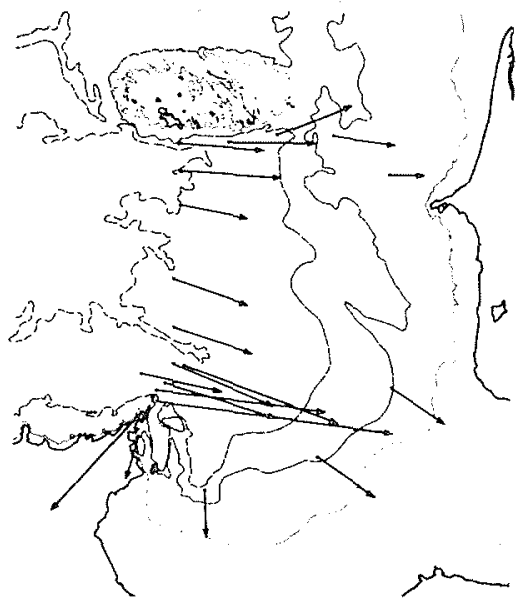

3H AVANT PM A SAINT SERVAN

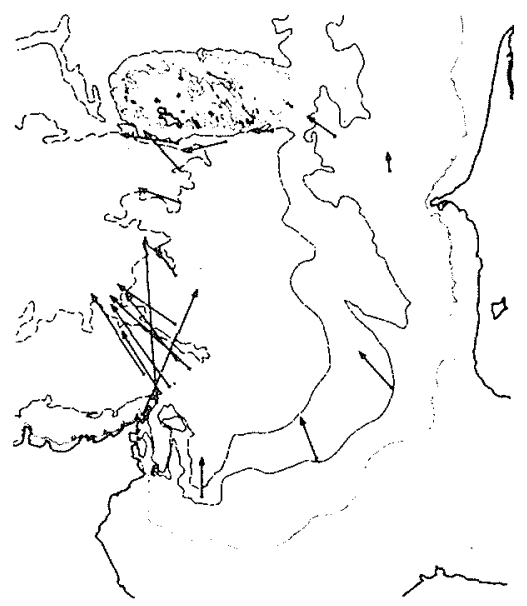

3H APRES PM A SAINT SERVAN

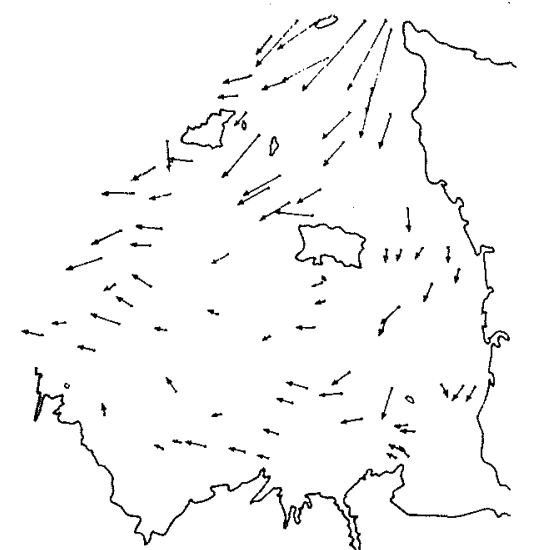

6H AVANT PM A SAINT SERVAN

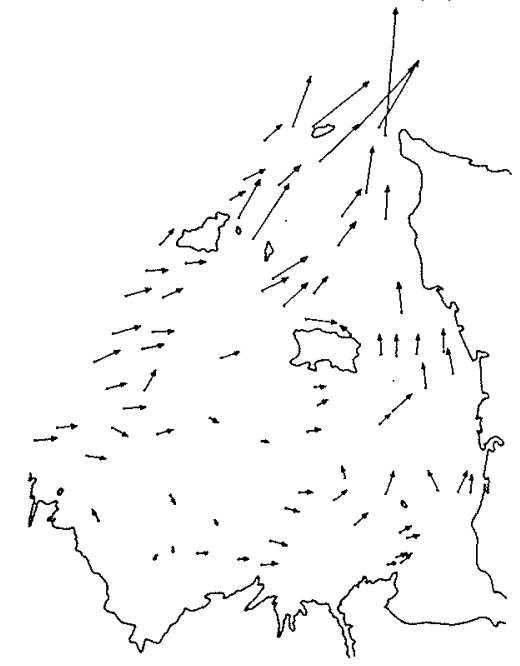

A PM A SAINT SERVAN

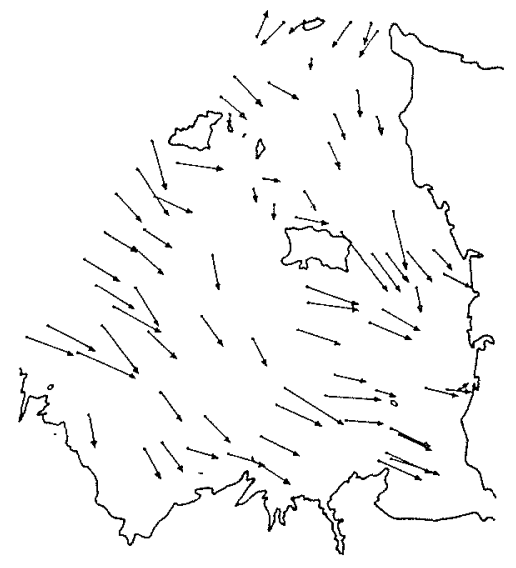

3H AVANT PM A SAINT SERVAN

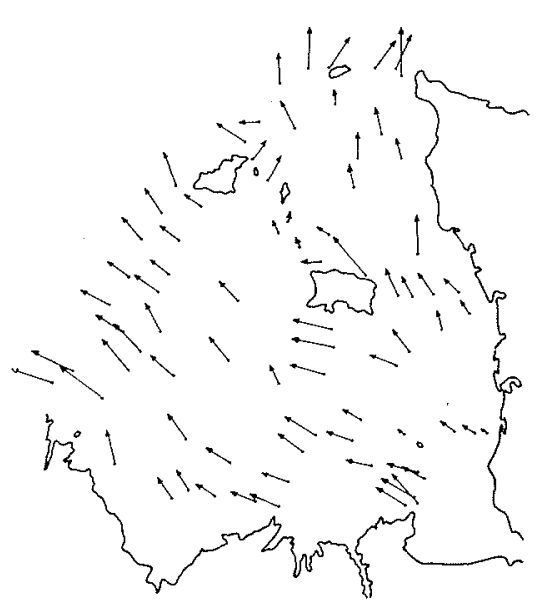

3H APRES PM A SAINT SERVAN 18/ Champs de courants en marée moyenne (coefficient 72)
dans la baie du Mont-Saint-Michel (d'après les campagnes S.E.U.M. de 1953 à 1954)

19/ Champs de courants en vive-eau (coefflcient 95)

dans le golfe de Saint-Malo (d'après les campagnes S.E.U.M. de 1957 à 1959) 

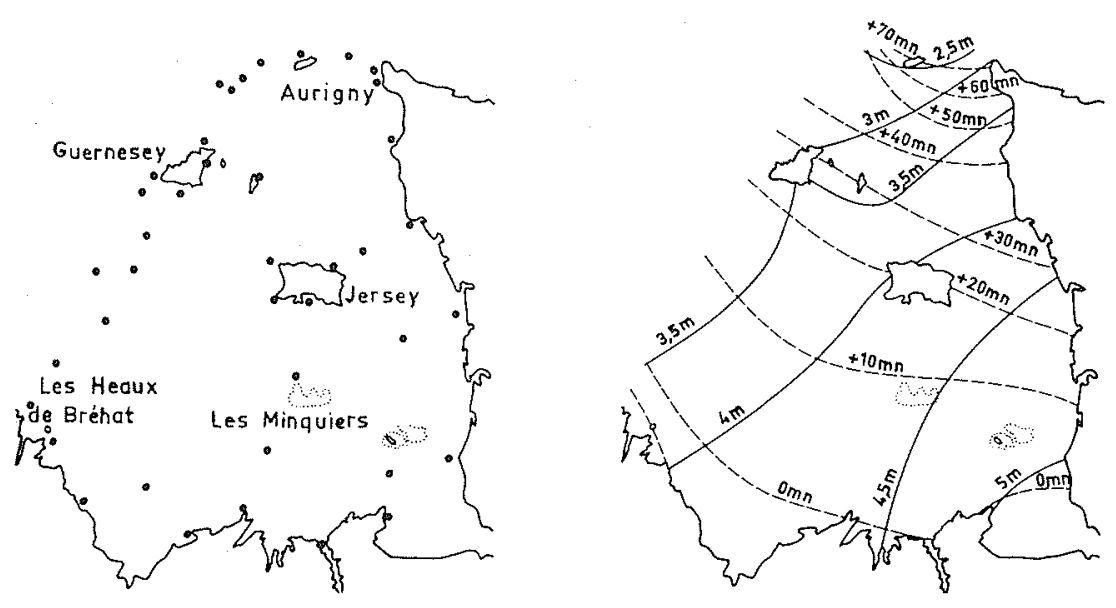

CARTE DES POINTS DE MESURES

$\overrightarrow{\vec{v}}$

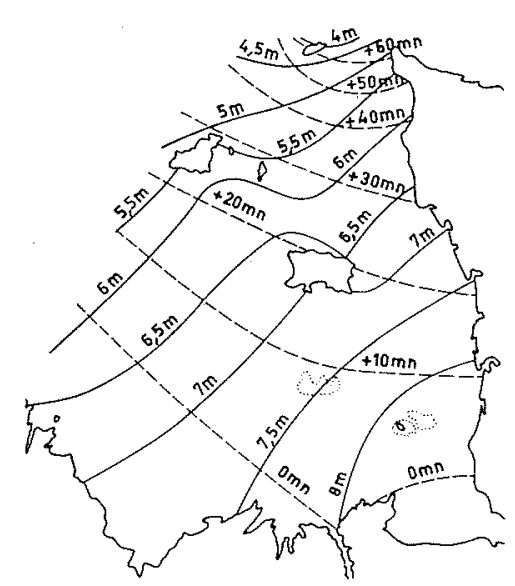

COEFFICIENT 70

Lignes d"egal marnage

Lignes cotidales de P.M

\section{COEFFICIENT 4.5}

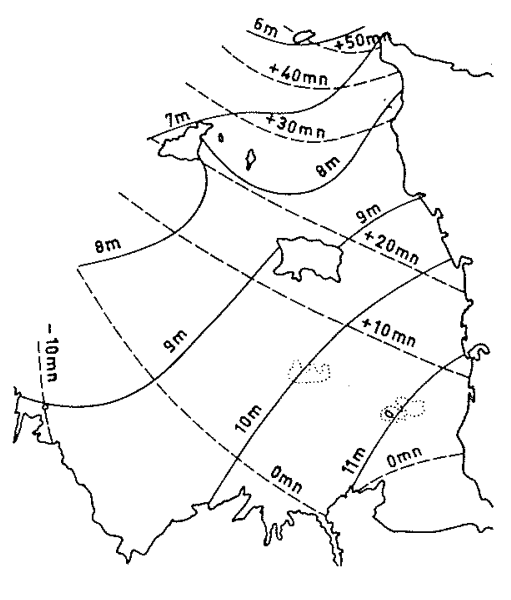

COEFFICIENT 95

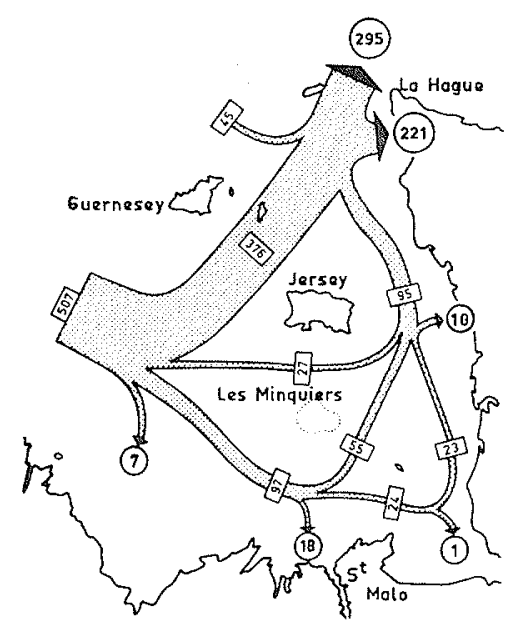

ENERGIE ACTIVE EN VIVE-EAU

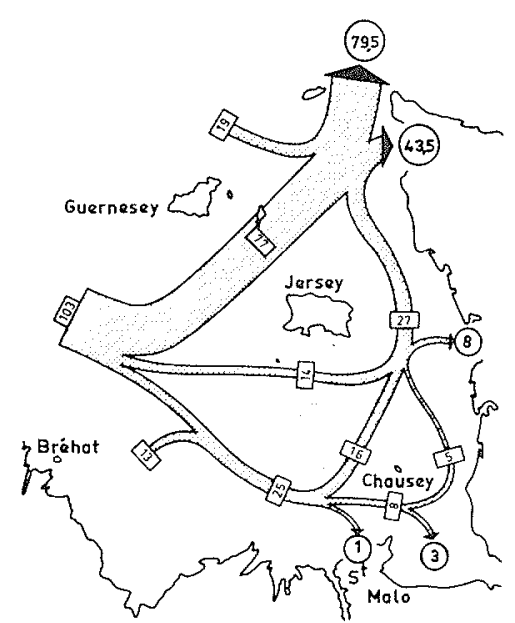

ENERGIE ACTIVEE EN MORTE-EAU

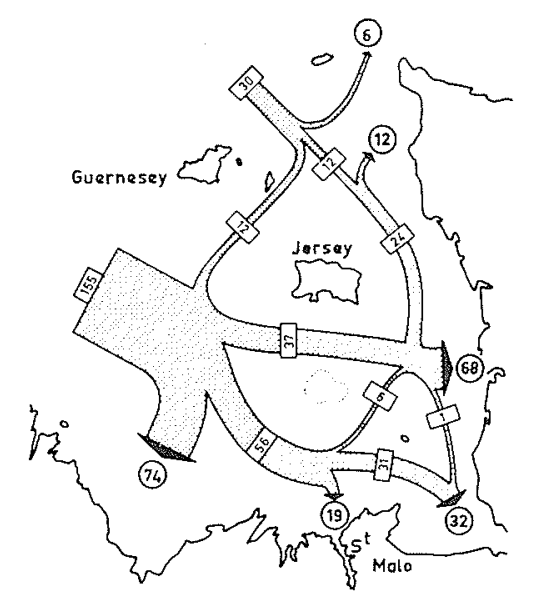

ENERGIE REACTIVE EN MORTE-EAU

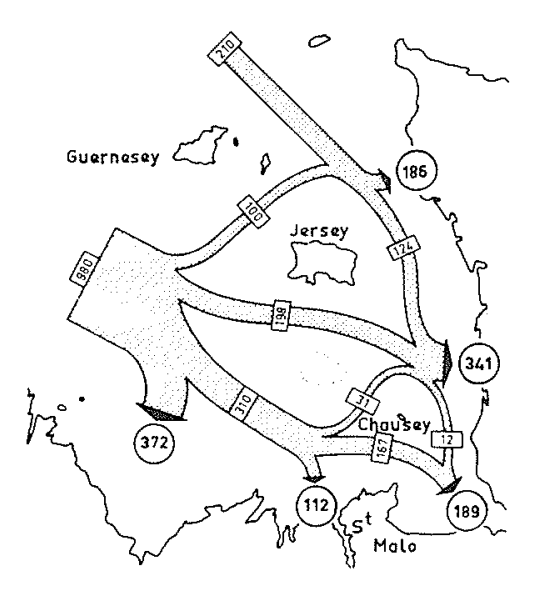

ENERGIE REACTIVE EN VIVE-EAU

(1) Energie consommée en GWh par marée

[2] Energie transportée en GWh par marée 

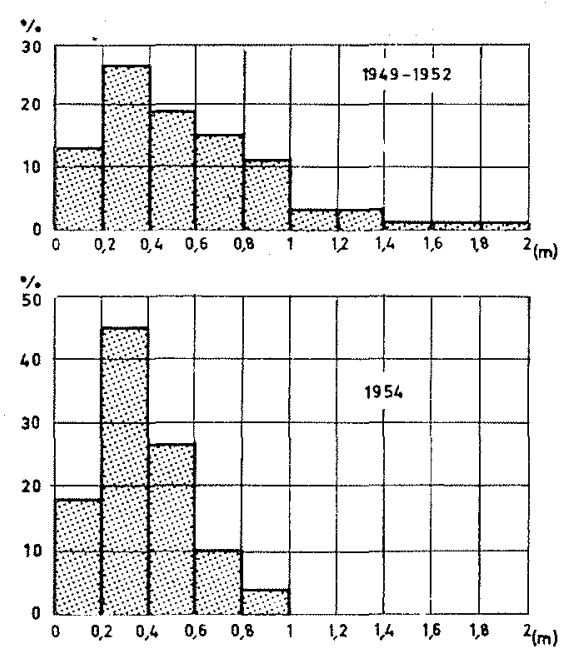

HAUTEURS MOYENNES

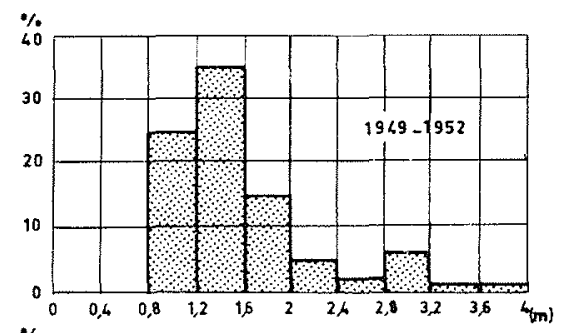

$\%$

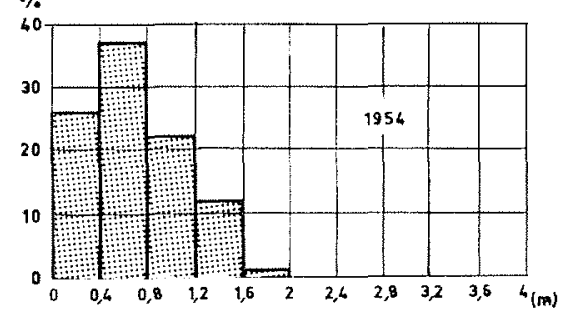

hauteurs maximales

22/ Mesure de houle à 1 mille au sud du phare de Chausey de mai 1949 à février 1952 et de juillet à novembre 1954
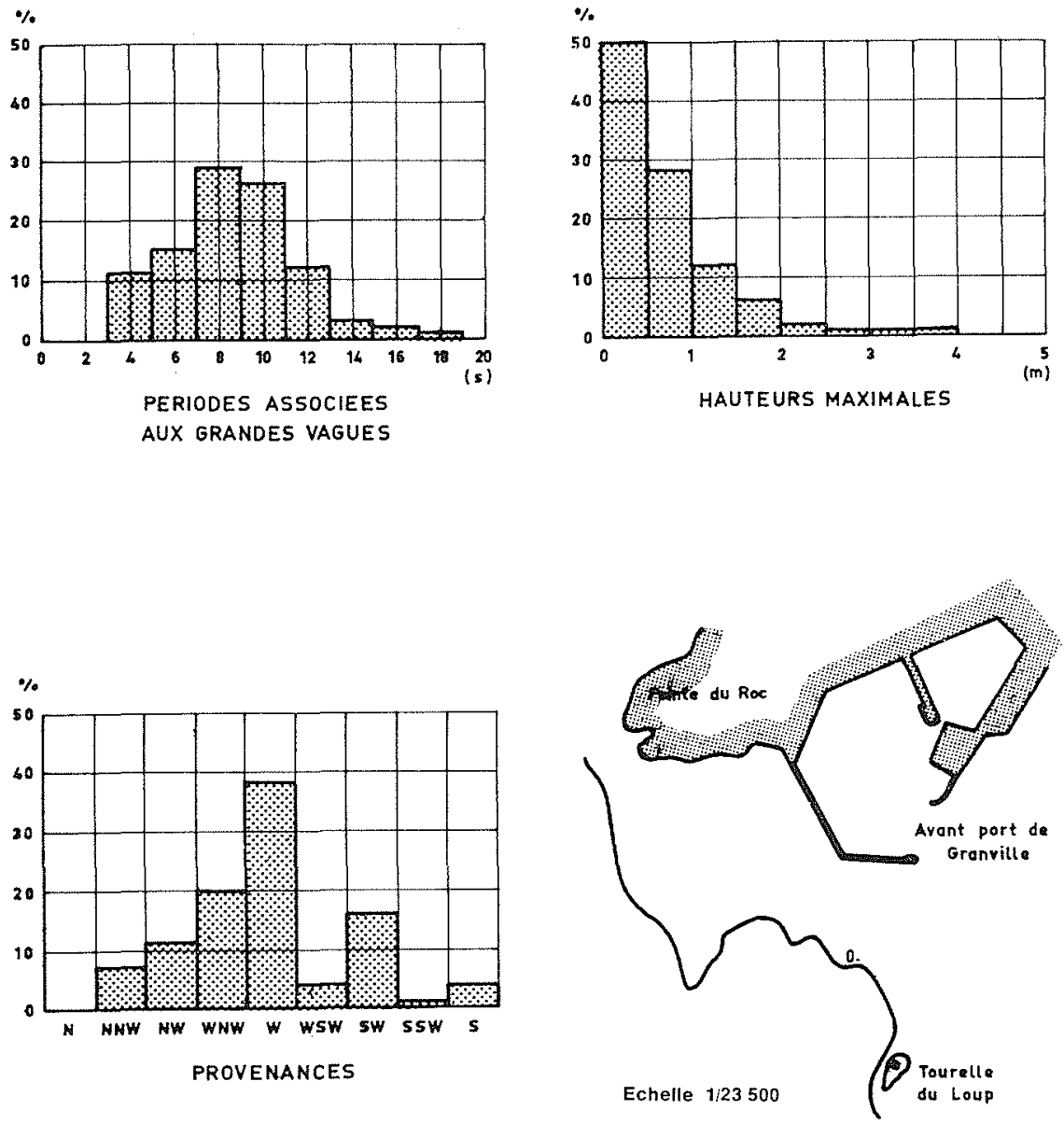

23/ Mesure de houle à la Tourelle-du-Loup (Granville) de juin 1965 à juillet 1966 
mêmes coefficients $[31,217 c]$. La figure 19 donne un aperçu des résultats obtenus.

\subsubsection{Les MESURES dE MARÉES}

Les premières mesures de marées dans la baie du Mont-Saint-Michel datent de 1953-54, lors des premières mesures de courants; par la suite, pour les besoins du modèle réduit, le S.E.U.M. entreprit, parallèlement aux mesures de courants, c'est-à-dire en 1957-58-59, une campagne de mesures de marées dans le golfe de Saint-Malo, à l'aide de marégraphes autonomes (35 points) installés à terre ou immergés, ou par lecture à des échelles de marée ( 6 points) toutes les $15 \mathrm{mn}$ de jour. Le principe de fonctionnement des marégraphes autonomes consistait à photographier toutes les $10 \mathrm{mn}$ un manomètre et un chronomètre. Les durées des observations en chaque point étaient de l'ordre de 2 à 6 mois (15 mois à Saint-Servan).

Ces relevés ont permis de relier les marnages locaux aux coefficients de marée, déterminer les déphasages entre les différents points du golfe de Saint-Malo, et donc d'établir les cartes de lignes d'égal marnage et cotidales [32, 217b] (fig. 20).

\subsubsection{Les calculs évergétiques}

La densité des mesures de niveau d'eau et de courants a permis d'entreprendre l'étude énergétique de la marée dans le golfe de Saint-Malo. A travers une section verticale $S$ de la mer, la puissance transmise en fonction du temps est :

$$
\vec{P}=\int_{S} \rho g H \vec{V} d S
$$

en désignant par :

$\vec{V}$ le vecteur vitesse;

$H$ la charge par unité de poids ;

$$
H=(V \cdot / 2 g)+(p / \rho g)+z \text {; }
$$

$p$ la pression à la cote $z$ comptée positivement audessus de la surface libre:

p la masse volumique du fluide ;

$g$ l'accélération de la pesanteur.

En désignant par $\zeta(x, y, t)$ la cote de la surface libre, on obtient :

$$
\vec{P}(l)=\int_{N} \rho\left(g \zeta+1 / 2 V^{2}\right) \vec{V} d S
$$

somme de l'énergie potentielle et de l'énergie cinétique (cette dernière étant en général négligeable, sauf au raz Blanchart).

Dans ces conditions la puissance transmise se trouve être de la forme :

$$
\overleftrightarrow{P}=\rho g \bar{c} \vec{Q}
$$

en désignant par :

$\vec{Q}$ le débit à travers la section $S$;

$\bar{\zeta}$ la valeur moyenne de la cote de la surface libre le long de la trace horizontale du profil de la section considérée.

Comme pratiquement $\bar{\zeta}$ et $Q$ sont des fonctions sinusoïdales du temps, on voit apparaître l'analogie avec la puissance d'un circuit électrique; en particulier on peut, dans ces conditions, définir les puissances actives et réactives passant au travers d'un profil $[28,38,217 d]$.

La détermination des puissances actives sur un contour permet d'arriver au bilan énergétique du domaine considéré (fig. 21). En l'absence de toute usine marémotrice, et en dehors de l'énergie correspondant au travail du potentiel astronomique, cette énergie est consommée par frottement sur le fond de la mer. Au fond des golfes elle est en général faible : la marée et le courant sont presque en quadrature; par conséquent l'énergie réactive y est grande.

L'étude des différentes composantes de l'énergie réactive montre que celle-ci est plus riche que son homologue électrique; en plus de l'énergie capacitive, qui correspond au remplissage des baies et des golfes, et de l'énergie d'inertie dans les zones à fortes vitesses, il apparaît de nouvelles énergies réactives : d'une part astronomique, due au déphasage entre le potentiel astronomique et la marée et, d'autre part, due aux rotations du vecteur vitesse du fait de la rotation de la Terre.

L'intérêt de ces considérations est la connaissance des importances relatives des différents composants du bilan énergétique de la marée et, par conséquent, la possibilité d'estimer quelle pourrait être la perturbation apportée par une usine marémotrice aux flux énergétiques.

\subsubsection{La houle}

\subsubsection{LES MESURES ET LEUR ANALYSE}

\subsection{Les mesures du S.E.U.M.}

Les premières mesures de houle du S.E.U.M. datent de mai 1949 à février 1952. Pendant cette époque 600 enregistrements journaliers furent exécutés à 1 mille au sud du phare de Chausey par fond de $-20 \mathrm{~m}$; ils furent complétés par 175 enregistrements bi-quotidiens d'environ $15 \mathrm{mn}$, entre juillet et décembre 1954, par fonds de $-5 \mathrm{~m}$.

Les enregistrements de 1949-52 et 1959 furent analysés par le S.E.U.M. [211] en étudiant, pour chacun d'eux, les hauteurs moyennes et maximales et la période moyenne (les notions hauteur et période significatives étaient ignorées à l'époque) et les observations de vent à la pointe du Roc. La conclusion est que les hauteurs moyennes et maximales se répartissent assez bien suivant la loi de Rayleigh :

$$
p(H)=\frac{H}{\left.H_{1}\right)^{2}} e^{-1 / 2\left(H / H_{0}\right)^{2}}
$$

avec $H_{0}=0,32 \mathrm{~m}$ pour 1954

$$
H_{0}=0,47 \mathrm{~m} \text { pour 1949-52; }
$$

pour les hauteurs moyennes et le double pour les hauteurs maximales. Il en résulte les valeurs intéressantes suivantes:

$$
\begin{aligned}
& \text { - probabilité } 0,01 \text { pour } H \geqslant 1,9 \mathrm{~m} \\
& \text { - probabilité } 0,001 \text { pour } H \geqslant 2,3 \mathrm{~m}
\end{aligned}
$$

L'étude des enregistrements de 1954 permit d'estimer statistiquement les hauteurs de houle maximale et moyenne (fig. 22). Pour les deux campagnes, les houles 

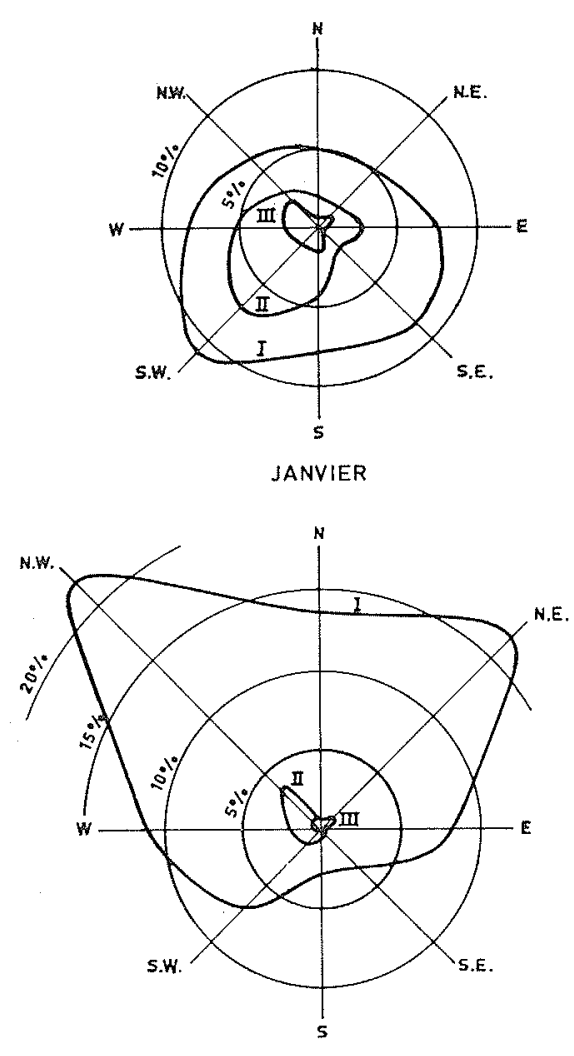

JUILLET

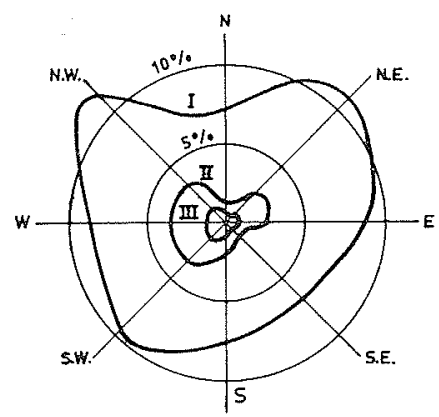

MOYENNE ANNUELLE
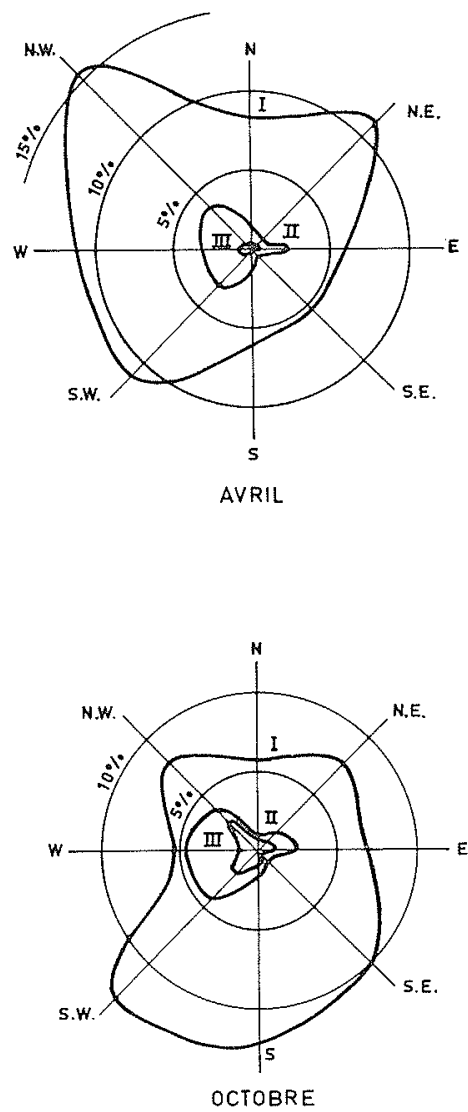

24 Force des vents au sommet du Grand-Mont (altitude $40 \mathrm{~m}$ ) sur la Grande-lle de Chausey (d'après J. Rouch)

les plus probables ont une amplitude moyenne de $0,3 \mathrm{~m}$; du fait que les mesures de 1954 ne couvrent qu'une saison, les valeurs enregistrées sont plus faibles qu'en 1949/52. Les hauteurs maximales observées atteignent $4 \mathrm{~m}$. Les périodes sont courtes, 3 à $7 \mathrm{~s}$, cette derniere valeur correspondant aux hauteurs maximales; il existe aussi des houles longues du secteur ouest, de période 10 à $20 \mathrm{~s}$, mais de hauteur en général inférieure à $1 \mathrm{~m}$.

\subsection{Les mesures du L.N.H.}

La deuxième série de mesures eut lieu de juin 1956 à avril 1960 au sud des îles Chausey et au nord de la pointe du Grouin; pour ces mesures, était utilisé un houlographe autonome du Laboratoire National d'Hydraulique. 58 films furent enregistrés; leur analyse sta- tistique est illustrée par les figures des planches 1 à 4; elle sera commentée plus loin $\left({ }^{3}\right)$.

Signalons enfin une troisième campagne de mesures, effectuée par le Laboratoire National d'Hydraulique [219], pour les besoins de l'étude du port de Granville; les résultats de l'analyse statistique sont présentés sur la figure 23 et les planches 5 et 6 , ils corroborent les précédents, en particulier la plus forte hauteur enregistrée est $4,3 \mathrm{~m}$, période $7,5 \mathrm{~s}$ (le 10 décembre 1965). Les "provenances " proviennent des observations au sémaphore de la Marine à la pointe du Roc du $1^{\text {er janvier au }}$ 31 juillet 1966. Ce sont les seules mesures de direction disponibles concernant la baie du Mont-Saint-Michel. Dans cette région, les plus fortes houles proviennent de W.-S.-W. et ont une période comprise entre 7 et $9 \mathrm{~s}$.

(3) On trouvera les planches citées à la fin de l'article. 


\subsection{Les mesures de vent}

La figure 24 présente les roses des vents mesurées à Chausey. L'autre source d'information locale, le sémaphore de la Marine de la pointe du Roc à Granville, conduit à la même conclusion :

- les vents violents proviennent de l'W.-S.-W. (maximum $25 \mathrm{~m} / \mathrm{s}$ );

- les vents faibles du secteur N.-N.-E. (maximum 15 $\mathrm{m} / \mathrm{s})$.

\subsubsection{LES INTERPRÉTATIONS PREVISIONNELLES SUR LA HOULE}

Une première étude de la houle fut demandée à SoGREAH par le S.E.U.M. en 1956 [208e]; elle concernait l'estimation des houles probables compte tenu des vents sur la grande île de Chausey (fig. 24) et des fetchs. Les vents prédominants sont du N.-W. et du N.-E. (printemps et été); les vents forts soufflent du N.-W. au S. $-W$. avec des vitesses moyennes de l'ordre de 15 à $30 \mathrm{~km} / \mathrm{h}$, sauf pour l'ouest $(75$ à $80 \mathrm{~km} / \mathrm{h})$. Le secteur par lequel peut pénétrer la houle du large, pour aborder la ligne Chausey-Grouin, est très limité (10 à 25") compte tenu de l'écran que constitue le plateau des Minquiers. Dans ces conditions, il semble que la houle maximale du site aurait une période de 6 à $7,5 \mathrm{~s}$ et une hauteur significative de l'ordre de $4 \mathrm{~m}$.

Une première esquisse de la détermination, par la méthode de Tukey, des spectres d'énergie d'une quinzaine d'enregistrements de houle [208k] a montré que la répartition des cotes de la mer est gaussienne et que donc les théories Longuet-Higgins et Cartwright sont applicables; l'étude des corrélations entre les caractéristiques spectrales et les paramètres météorologiques reste à faire. Quelques tentatives de reproduction de ces houles en canal ont été faites [208l].

En définitive, il est possible d'énoncer les conclusions suivantes :

- les houles les plus probables ont une hauteur de l'ordre de $0,6 \mathrm{~m}$ et une période assez longue, 9 à $12 \mathrm{~s}$, atteignant parfois la vingtaine de secondes (planche 2);

- les houles les plus fortes (de 4 à $5 \mathrm{~m}$ ) ont une période de l'ordre de 9 à $12 \mathrm{~s}$, elles proviennent de l'ouest; des vagues de près de $5 \mathrm{~m}$ avec une période de $18 \mathrm{~s}$ ont été enregistrées:

- la "houle décennale» est $6 \mathrm{~m}$ (ce qui est effectivement observé);

- il faut s'attendre à des vagues maximales de 5 à $6 \mathrm{~m}$, donc la « hauteur significative de projet 》 est $4 \mathrm{~m}$.

Enfin les planches 7 à 9 présentent quelques plans de vagues. Elles montrent que la houle ouest aborde l'usine frontalement, qu'une convergence de la houle de N.-W. est à craindre vers le centre de la digue pointe du Grouin-Chausey.

\subsubsection{L'iNflueNCE DE LA HOULE SUR L.e COMPORTEMENT DES GROUPES MAREMOTEURS}

\subsection{Les études E.D.F.}

Le cas d'une usine marémotrice soumise de plein fouet à la houle pose le problème de l'influence des fluctuations de niveaux et pressions dus à la houle de- vant l'usine. Ce problème a été examiné d'une part à E.D.F. par des essais sur «microréseau » [19] et d'autre part à SOgReah par des calculs hydromécaniques des oscillations du couple moteur des groupes [208c, 208h, $208 i]$.

Le microréseau servit à une étude paramétrique des phénomènes dans les domaines suivants :

- houle maximale $4 \mathrm{~m}, 6 \mathrm{~s}$ et $2 \mathrm{~m}, 12 \mathrm{~s}$;

- période de la houle de 0,5 à $12 \mathrm{~s}$;

- houle agissant en phase sur la totalité des groupes et en opposition de phase sur chaque moitié d'usine:

- avec ou sans régulateur de tension;

- variation de 3 à l'infini du rapport entre les puissances de court-circuit sur le réseau $225 \mathrm{~kW}$ à Chausey d'une part du réseau et d'autre part des groupes; la valeur 3 , estimée la plus probable pour les années de fonctionnement de l'usine (1975), fut spécialement étudiée pour une usine de $12 \mathrm{GW}$ équipée de groupes de constante inertie 1,55 et $5 \mathrm{~s}$ (on appelle constante d'inertie d'un groupe le rapport entre son énergie cinétique de rotation et sa puissance nominale).

Dans l'hypothèse défavorable où une grande partie de l'usine oscille en phase, les perturbations de tension ne seraient pas négligeables sur le jeu de barre de l'usine, même en supposant les groupes munis de régulateur de tension $(6 \%$ à $9 \%$ ). Mais, dans le cas plus probable où les effets de la houle se compenseraient sur un grand nombre de groupes, la perturbation de tension sera faible. Dans le cas pessimiste, forte houle en phase, avec une bonne régulation de tension les oscillations de tension seront de l'ordre de $\pm 2 \%$ pour les houles de périodes 6 et $12 \mathrm{~s}$.

L'influence de la houle se traduit aussi par des oscillations des courants induits, provoquant un suréchauffement des enroulements, qui, au contraire des oscillations de tension, ne sont pas réduites par les régulateurs de tension. Cet échauffement peut coriespondre à une surintensité de $8 \%$ environ; il faudrait en tenir compte dans les données constructives des alternateurs. En revanche la mise en service de régulateurs de tension sur les groupes éliminera tout risque du fait de la houle de perte de synchronisme entre les groupes et le réseau.

Dans le même ordre d'idée, rappelons l'étude sur microréseau de l'effet de la houle de période $6 \mathrm{~s}$, hauteur $1 \mathrm{~m}$, dans le cas de l'usine de la Rance [102]; elle a montré l'absence de danger pour la stabilité des groupes. L'étude de la résonance du réseau a aussi montré que celle-ci disparaît presque complètement lorsque la fréquence excitatrice est moindre que $1,5 \mathrm{~Hz}$; la résonance n'est donc pas à craindre pour la houle de fréquence comprise entre 0,25 et $0,1 \mathrm{~Hz}$ ( 4 et $10 \mathrm{~s}$ ).

\subsection{Les études SOGREAH}

En 1953 le S.E.U.M. demanda au Laboratoire Dauphinois d'Hydraulique (Grenoble) d'examiner théoriquement et en modèle réduit les effets de la houle sur le parement aval de l'usine des îles Chausey. Les principales conclusions sont les suivantes; en houle régulière $[208 a, 208 b, 208 c]$ :

- les formules théoriques de calcul de la pression dans le clapotis sont valables; 
- une digue immergée devant l'ouvrage, à des distances de l'ordre de 50 à $100 \mathrm{~m}$, est inefficace, même néfaste;

- les dépressions prédominent sur les surpressions et il apparaît un dédoublement de la période;

- le débit des groupes a peu d'influence sur les fluctuations de pression.

L'action de la houle irrégulière se manifeste par l'effet de gifle; les essais en modèle réduit [208d] montrent l'intérêt d'une fosse devant l'usine, permettant d'enfoncer les groupes.

Des recherches sur les dispositifs permettant de réduire le clapotis devant l'usine [208g] ont montré que les talus inclinés sont peu efficaces; en revanche, les dispositifs anti-réfléchissants résonnants (voile perforé), ou absorbants (talus rugueux), sur le toit de l'usine, seraient efficaces. L'étude du coefficient de réflexion de la houle fut reprise avec le parement du caisson usine du projet de juin 1957 [208j]; un type de parement a été défini; il donne un coefficient de réflexion de $85 \%$ quelque soit le niveau de la mer.

L'effet des fluctuations de pression sur la marche des groupes a été examiné sous la forme de l'établissement de méthodes de calcul des variations du couple d'un groupe sous l'effet des fluctuations de la charge [208h] et des pulsations de la puissance sous l'effet de la houle [208i]. Les rapports présentent les outils théoriques de base sous forme d'abaques adimensionnels pour calculer les fluctuations de puissance dans les divers cas de fonctionnement de l'usine.

\subsection{Les études hydrauliques}

\subsubsection{Les difficultés}

Les études hydrauliques du projet de l'usine des îles Chausey ont, dès le début, mis les ingénieurs devant des difficultés nouvelles à l'échelle de l'entreprise. Au début des années 1950, seuls les modèles réduits physiques étaient capables de répondre à la question fondamentale, objectif des études hydrauliques: quelles seront les modifications de la marée, d'une part au cours de la construction de l'ouvrage, d'autre part dues au fonctionnement de l'usine? La première partie de la question intéresse et conditionne l'exécution des travaux, donc une grande partie du prix de l'usine. L'ensemble de la question est essentielle au niveau du calcul de l'énergie produite par l'usine. Elle intéresse aussi les océanographes, les navigateurs et tous ceux qui s'occupent de l'aménagement du territoire, puisque les lois de niveau et de courant seront légèrement changées.

Or, il ne s'agissait pas de se contenter de construire des modèles réduits ne représentant que quelques kilo. mètres carré de surface marine. A priori, par son aspect énergétique, la dizaine de gigawatts, le projet intéresse un domaine qui s'étend hors du golfe de Saint-Malo, c'est-à-dire la zone entre Bréhat et le cap de la Hague où l'onde-marée accuse une puissance de $25 \mathrm{GW}$ en vive-eau [5]. Le projet est à l'échelle de la Manche entière où, en vive-eau, l'onde-marée apporte $180 \mathrm{GW}$ [6]. Mais la représentation sur modèle réduit d'une zone marine de cette importance soulève une difficulté originale; parmi les différentes forces qui gouvernent le mé- canisme de la marée, la force de Coriolis, due à la rotation de la Terre, n'est plus négligeable; par exemple, à la latitude $45^{\circ}$, l'accélération de Coriolis sur un courant de $1 \mathrm{~m} / \mathrm{s}$ est égale à celle résultant d'une pente de surface libre de $1 / 100000$, c'est-à-dire une pente équivalente à celle de l'onde-marée en Manche : $10 \mathrm{~m}$ d'amplitude sur $1000 \mathrm{~km}$ de longueur d'onde.

Or, représenter la force de Coriolis sur modèle réduit hydraulique, pose un problème difficile. Au niveau de la reproduction de la totalité de la Manche, il n'est pas possible d'esquiver ce problème; mais ne peut-on pas se le permettre pour des modèles d'emprise plus restreinte, par exemple pour les modèles d'étude des conditions hydrauliques aux abords de l'usine. Pour examiner cette question fut créé en 1954 un comité scientifique dénommé le "Pool Coriolis", avec le concours des représentants de la Faculté des Sciences de Grenoble, du Service Central Hydrographique de la Marine, du Laboratoire d'Océanographie Physique du Museum d'Histoire Naturelle, du Laboratoire Dauphinois d'Hydraulique (SOGREAH).

\subsubsection{Les modèles réduits}

Un premier modèle réduit de la Manche entière fut construit en 1954 au Laboratoire National d'Hydraulique $[217 a]$; ne tournant pas, il ne pouvait prétendre à une représentation correcte à l'onde-marée [38]. Toutefois, malgré ses échelles très petites (1/50 000 en plan, $1 / 500$ en hauteur), il permit d'énoncer quelques règles générales sur le programme de fermeture des bassins. Le deuxième modèle de la Manche, avec les mêmes échelles que le précédent, fut réalisé sur la plateforme tournante de $14 \mathrm{~m}$ de diamètre (fig. 25), construite par Sogreah pour le compte de l'Université de Grenoble [28g]. Cette réalisation, techniquement des plus remarquables, permit une reproduction très fidèle de la marée en Manche; de ce fait, elle constitue un outil de recherche fondamental dans ce domaine. Ce modèle (fig. 26) a déjà donné une première approximation du "remous» des digues (réduction de $50 \mathrm{~cm}$ du marnage de la marée de vive-eau), puis du remous dû au fonctionnement des groupes de l'usine marémotrice projeté à cette époque [36].

Les modèles d'ensemble de la Manche ont montré que, pour étudier sur modèle réduit les phases de construction de l'usine, il suffisait de représenter le golfe de Saint-Malo. Le modèle devait-il être tournant ou non? L'étude de cette question fut abordée sur modèles schématiques montés sur une petite plateforme tournante de $4 \mathrm{~m}$ de diamètre [36]. La réponse négative [21] tient au fait que l'onde-marée dans le golfe de Saint-Malo est le résultat non seulement de l'effet de la force de Coriolis qui, responsable de la formation d'une onde de Kelvin dans la Manche, accentue les marnages sur les côtes françaises, mais aussi de la diffraction de l'onde autour du musoir que constitue le cap de la Hague, de sa réflexion sur la barrière que constitue la presqu'ile du Cotentin, du frottement sur la profondeur faible et enfin de la topographie compliquée du site. Il en résulte que l'effet de la force de Coriolis perd ici de son importance et qu'un modèle qui l'ignore peut, cependant, assez bien représenter le phénomène hydraulique, et par suite ses modifications par la présence de l'usine. 


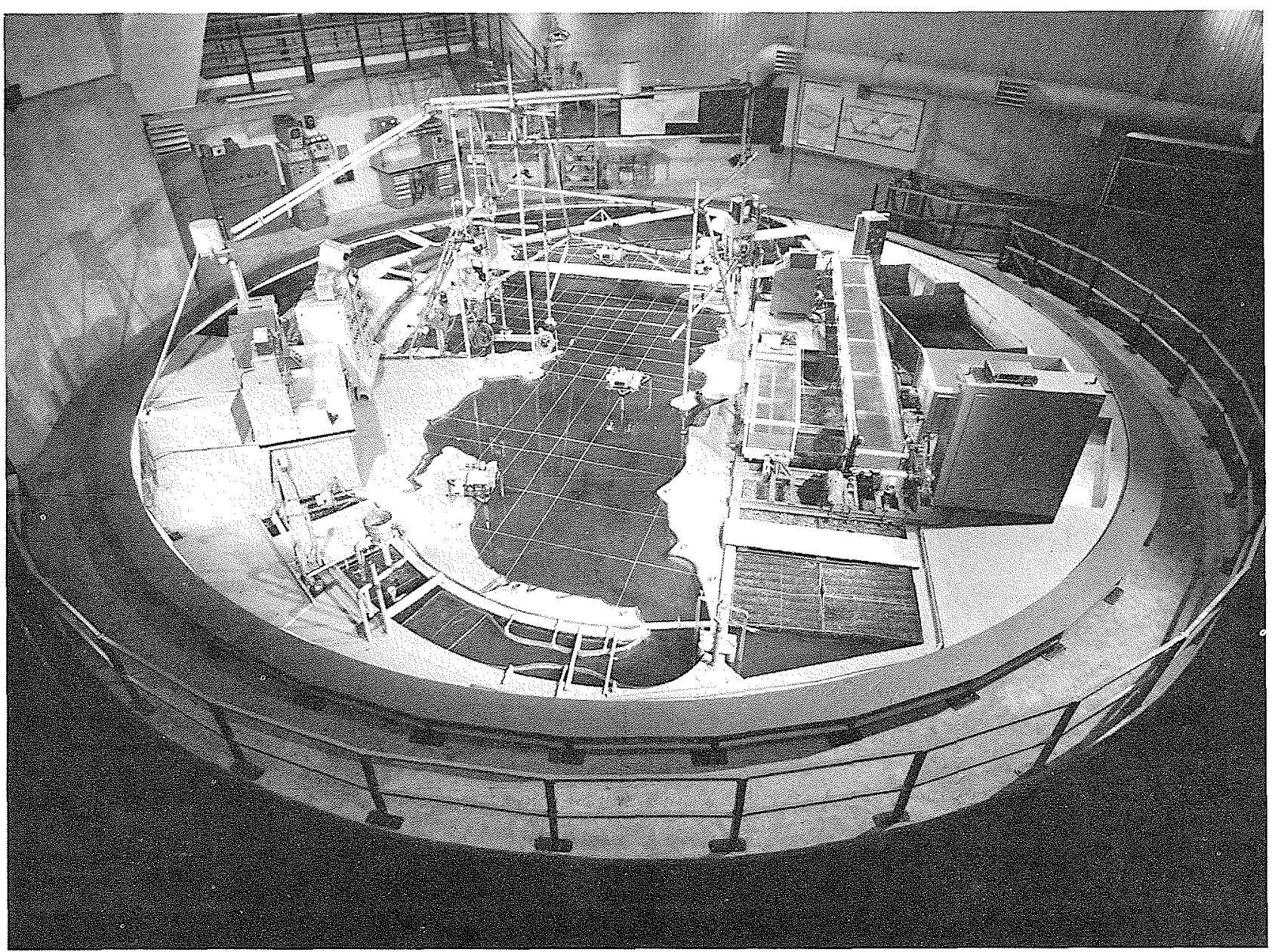

25/ Le modèle de la Manche sur la plate-forme tournante de l'Université de Grenoble

La construction du modèle non tournant représentant le golfe de Saint-Malo commença en 1957, avec les échelles de 1/2 500 en plan et 1/300 en hauteur; occupant une zone de $50 \times 60 \mathrm{~m}$, ce modèle fut, à son époque, le plus grand des modèles réduits du monde [217a]. La somme de mesures en nature qu'il nécessita pour son étalonnage est considérable (cf. point 3.1.1); mais cette contribution est des plus intéressante du point de vue de la connaissance océanographique du golfe de Saint-Malo $[217 b, 217 c, 217 d]$. L'étalonnage hydraulique du modèle se termina en 1964, mais l'étude fut interrompue avant que soient précisées les conditions hydrauliques au cours de la construction de l'usine.

Parmi les enseignements à tirer de cette entreprise, considérons ceux permettant de savoir s'il faudrait recommencer dix ans après. Actuellement, les modèles mathématiques éviteraient de construire un si grand modèle; il serait possible de se contenter de modèles locaux dont les conditions aux limites seraient, soit calculées, soit déduites de modèles physiques de grande emprise mais d'échelle plus petite, de l'ordre du $1 / 10000$ par exemple ou même, par des mesures très précises et nombreuses sur le modèle tournant, au $1 / 50000$. Le grand avantage serait de réduire le temps des essais.
Parallèlement aux modèles physiques, l'étude théorique de la marée avec application à la Manche connut à cette époque un grand essor, dû au dynamisme du Pool Coriolis $[11 c, 18,20,36]$; puis les succès des modèles physiques suscitèrent l'émulation des modèles mathématiques $[25,34]$. Aucune étude sédimentologique ne fut entreprise; or il est bien évident qu'au cours de la construction de l'usine, des problèmes de ce type se poseraient du fait de l'épaisse couche de sédiments qui recouvre la baie du Mont-Saint-Michel. De même n'a pas été regardée l'influence des déformations de la loi de niveau de la mer par rapport à une sinusoïde; or le premier harmonique de la marée vaut 7 à $8 \%$ du fondamental dans cette région.

\subsubsection{Les principaux résultats}

Les principaux résultats obtenus sur le modèle de la Manche non tournant $[216 b, 216 c]$ sont les suivants:

- la présence d'une digue de Chausey à Granville ou de Chausey à Bréhal n'a pas d'influence sur la marée ;

- la digue nord étant édifiée la première, la digue entre la pointe du Grouin et Chausey perturbe la marée dans la zone à l'est de la ligne Bréhat-Jersey- 


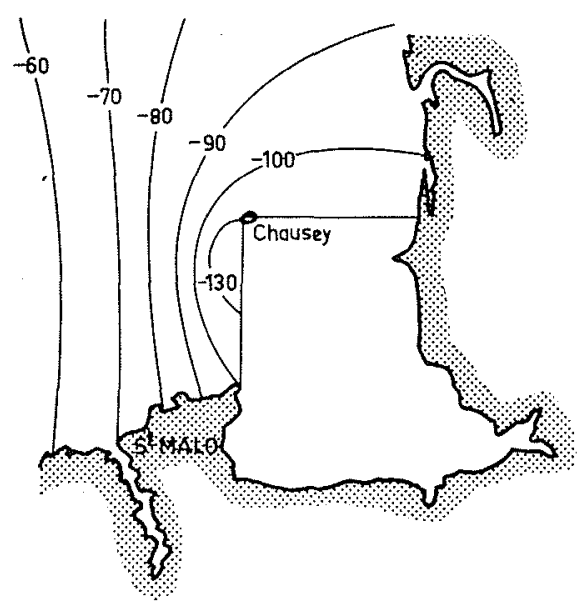

Débit maximal $400000 \mathrm{~m}^{3} / \mathrm{s}$

$(22 \mathrm{GW})$

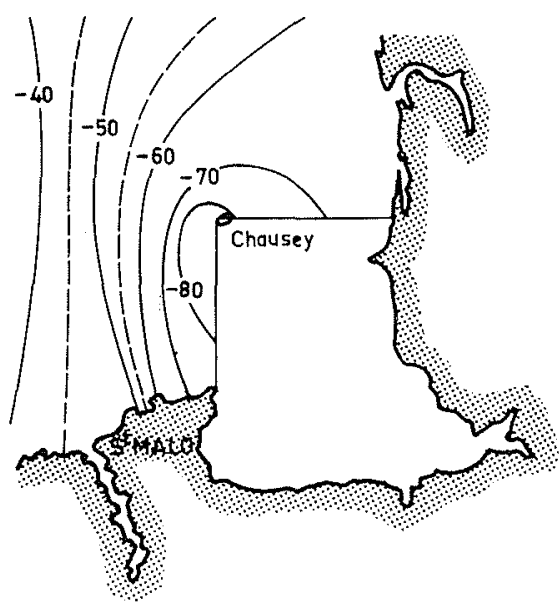

Débit maximal $200000 \mathrm{~m}^{3} / \mathrm{s}$

( $11 \mathrm{GW}$ )

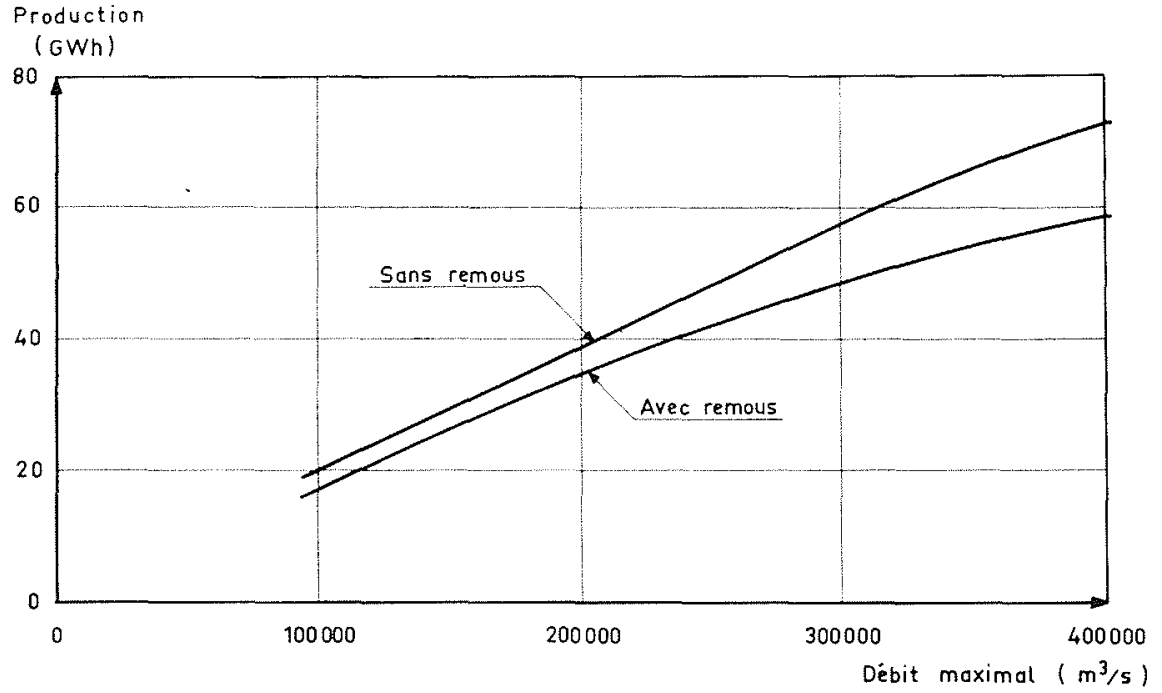

26/ Remous (en $\mathrm{m}$ ) du fonctlonnement de l'usine et effets sur la production pour une marée de vive-eau (coefficient 95)

Carteret; il s'agit d'une augmentation du marnage, qui est maximal au large de la pointe du Grouin $(50 \mathrm{~cm})$, et d'une diminution des courants de $0,5 \mathrm{~m} / \mathrm{s}$;

- si la digue nord n'est pas construite, la présence de la digue ouest est très perturbatrice: les courants augmentent de $1,5 \mathrm{~m} / \mathrm{s}$ sur la ligne ChauseyGranville.

L'étude des courants dans les brèches de fermeture des digues en cours de construction [216 l] montre que le courant atteint $5 \mathrm{~m} / \mathrm{s}$ lorsque la largeur de la brèche est de l'ordre de $1 \mathrm{~km}$. Le meilleur programme de travaux est d'édifier la digue Chausey-Bréhal, la direction de l'avancement étant indifférente, puis construire la digue pointe du Grouin-Chausey en commençant à partir du sud; cette façon de procéder permet de fermer les trois quarts de la distance Grouin-Chausey, avant de rencontrer dans la brèche des courants de $3 \mathrm{~m} / \mathrm{s}$ en vive-eau. Les conditions difficiles se situent au-delà : $5 \mathrm{~m} / \mathrm{s}$ pour une brèche de $2 \mathrm{~km}$ près de Chausey, $6 \mathrm{~m} / \mathrm{s}$ pour une brèche de $1 \mathrm{~km}$.
Si l'usine comporte deux bassins séparés par la digue reliant la pointe de Carolles au milieu de la distance pointe du Grouin-Chausey, il y a intérêt à édifier d'abord cette digue intermédiaire : lors de la fermeture du bassin Sud, les courants sont plus faibles que dans le cas de la fermeture du bassin total $(4,5 \mathrm{~m} / \mathrm{s}$ pour une brèche de $1 \mathrm{~km}$ ). De même il y a intérêt à construire d'abord la digue Chausey-Bréhal délimitant le bassin Nord.

Les premères études d'influence de l'usine effectuées, soit sur les modèles physiques [36], soit par les calculs numériques [27f], permirent d'obtenir les ordres de grandeur du remous de l'usine et, en particulier, la linéarité de l'effet avec le débit de l'usine, tout au moins jusqu'au débit de $400000 \mathrm{~m}^{3} / \mathrm{s}$ (correspondant à la puissance de $22 \mathrm{GW}$ ).

L'étude plus précise du remous de l'usine fut entreprise à nouveau, sur le modèle tournant de la Manche à Grenoble, avec les hypothèses soit d'une usine arrêtée, soit d'un fonctionnement de l'usine schématisé par un débit alternatif de phase variable avec la marée, donc 
pouvant simuler le turbinage, le pompage et les situations intermédiaires $[36,220]$. La figure 26 donne les lignes d'égale perturbation de la marée dans le cas où le débit est en retard d'une demi-période de marée, déphasage correspondant au maximum de production d'énergie. Le calcul comparatif des productions avec ou sans remous permet de préciser l'influence énergétique.

Dans les conditions suivantes :

- bassin de $728 \mathrm{~km}^{2}$ à la cote $+13,5 \mathrm{~m} \mathrm{CM}$;

- groupes identiques au groupe-bulbe expérimental de Saint-Malo;

- marée sinusoïdale de coefficient 95 (marnage $11 \mathrm{~m}$ );

- cycle à double effet avec pompage;

l'effet est résumé dans le tableau 7.

\begin{tabular}{|c|c|c|c|c|}
\hline \multicolumn{5}{|r|}{ Tableau 7} \\
\hline \multirow{3}{*}{$\begin{array}{l}\text { PUISSANCE } \\
\text { INSTALLEE } \\
(\mathrm{GW})\end{array}$} & \multirow{3}{*}{$\begin{array}{c}\text { DEBIT } \\
\text { WAXIMAL } \\
\left(\mathrm{m}^{3} / \mathrm{s}\right)\end{array}$} & \multicolumn{2}{|c|}{ PRODUCTION } & \multirow{3}{*}{$\begin{array}{c}\text { PERTE } \\
\text { ENERGETIQUE } \\
(\%)\end{array}$} \\
\hline & & $\begin{array}{l}\text { sans } \\
\text { remous }\end{array}$ & $\begin{array}{l}\text { avec } \\
\text { remous }\end{array}$ & \\
\hline & & $(G W h)$ & $(G W h)$ & \\
\hline 5,5 & 100000 & 20 & 18 & 10 \\
\hline 11 & 200000 & 39 & 35 & 11 \\
\hline 16 & 300000 & 57 & 49 & 15 \\
\hline 22 & 400000 & 72 & 58 & 20 \\
\hline
\end{tabular}

En conclusion, pour la puissance envisagée, la réduction de marnage sera de l'ordre du mètre devant l'usine et la perte d'énergie de plus de $10 \%$. Il n'est donc pas possible d'estimer la production énergétique de l'usine sans tenir compte du remous côté mer. Quel serait l'effet du remous côté bassin? La question n'a pas été examinée.

\subsection{Les études géologiques}

L'étude géologique du projet de l'usine marémotrice reçut un développement rarement égalé, à la mesure de l'ensemble de l'entreprise envisagée. Les difficultés à vaincre étaient considérables : il fallait créer un matériel spécifique et des méthodes inédites: mettre au point des engins océaniques, savoir compléter les informations des sondages par l'interprétation géologique basée sur la reconnaissance des affleurements rocheux naturels, ceci tout en étant soumis aux aléas des conditions climatiques.

\subsubsection{Mesures bathymétriques}

De juin à septembre 1957 et en septembre 1958, le S.E.U.M. effectua une série de sondages hydrographiques de la baie du Mont-Saint-Michel. Quatre cartes au
1/20000 furent établies, présentées dans le Système Lambert I et rapportées au NGF $(+6,60 \mathrm{~m} \mathrm{CM})$.

Les extraits de cartes présentés ici sont établis à partir des résultats de cette campagne, sauf ceux illustrant les mesures de champ de courant.

Il y aurait intérêt à poursuivre ces mesures sur une bande de $5 \mathrm{~km}$ plus à l'ouest et plus au nord.

\subsubsection{Mesures géophysiques}

En 1952-53, le S.E.U.M. demanda à la Compagnie Générale de Géophysique (50, rue Fabert, Paris-7 ${ }^{\circ}$ ) une série d'études du substratum de la baie. La première série d'essais sismique-réfraction en 1952 [202] mit en relief une distinction possible entre les schistes Briovérien (Précambrien supérieur) et les granites de Chausey (célérités respectivement inférieures à $3500 \mathrm{~m} / \mathrm{s}$ et supérieures à $4000 \mathrm{~m} / \mathrm{s}$ ), laissant ainsi entrevoir la possibilité d'une reconnaissance de la nature du substratum par la méthode sismique-réfraction. La campagne de 1953 [203, 205] consista à prospecter un couloir de $9 \mathrm{~km}$ de long et 1 à $2 \mathrm{~km}$ de large au S.-E. des îles Chausey; le principal résultat est la localisation du contact granites-schistes à $1,5 \mathrm{~km}$ au sud de la bordure de l'archipel. Il est à noter que, dans cette zone, les sondages ultérieurs n'ont pas décelé de granites. D'autres affleurements granitiques ont été décelés plus au S.-E, mais leur présence n'a pas été confirmée par les sondages.

En outre, la Compagnie Générale de Géophysique commença en 1952 [203] la détermination, par prospection électrique, de l'épaisseur des sédiments meubles dans l'archipel de Chausey (sables sur granites). La méthode utilisait le fait que ces terrains sont électriquement bien différenciés : la résistivité des sables imbibés d'eau de mer est au moins dix fois inférieure à celle des granites. 278 sondages électriques furent effectués. Les épaisseurs de sable (ou de granite altéré) sont de l'ordre de la dizaine de mètres à l'est et au nord de l'archipel, et de l'ordre de 1 à $3 \mathrm{~m}$ dans la partie centrale

\subsubsection{Mesures géologiques}

\subsubsection{NÉCESSITÉ ET CONTINGENCES D UNE ÉTUDE GEOLOGIQUE}

L'interprétation des résultats apparut incertaine: il n'y avait guère accord entre les données géologiques reçues (exprimées par la carte géologique en usage alors) et celles fournies par les sondages jusqu'en 1955. Il fut donc décidé d'entreprendre, avec la collaboration d'un géologue-conseil, une série de campagnes géologiques. De ce fait, à la lumière des cinq campagnes de 1955 à 1959, et le développement des nouvelles recherches de M. J. Graindor quant à la tectonique, les résultats géophysiques ont pris toute leur valeur.

\subsubsection{Méthodes et Moyens techniques}

Les sondages géologiques furent effectués, dans les roches indurées, par forage au trépan et extraction de carottes. Les premiers sondages commencèrent en 1955, de part et d'autre de l'alignement Granville-pointe du Grouin-Chausey, au moyen d'une plateforme autoélévatrice, «le Diplodocus» soutenue par quatre pieds ca- 


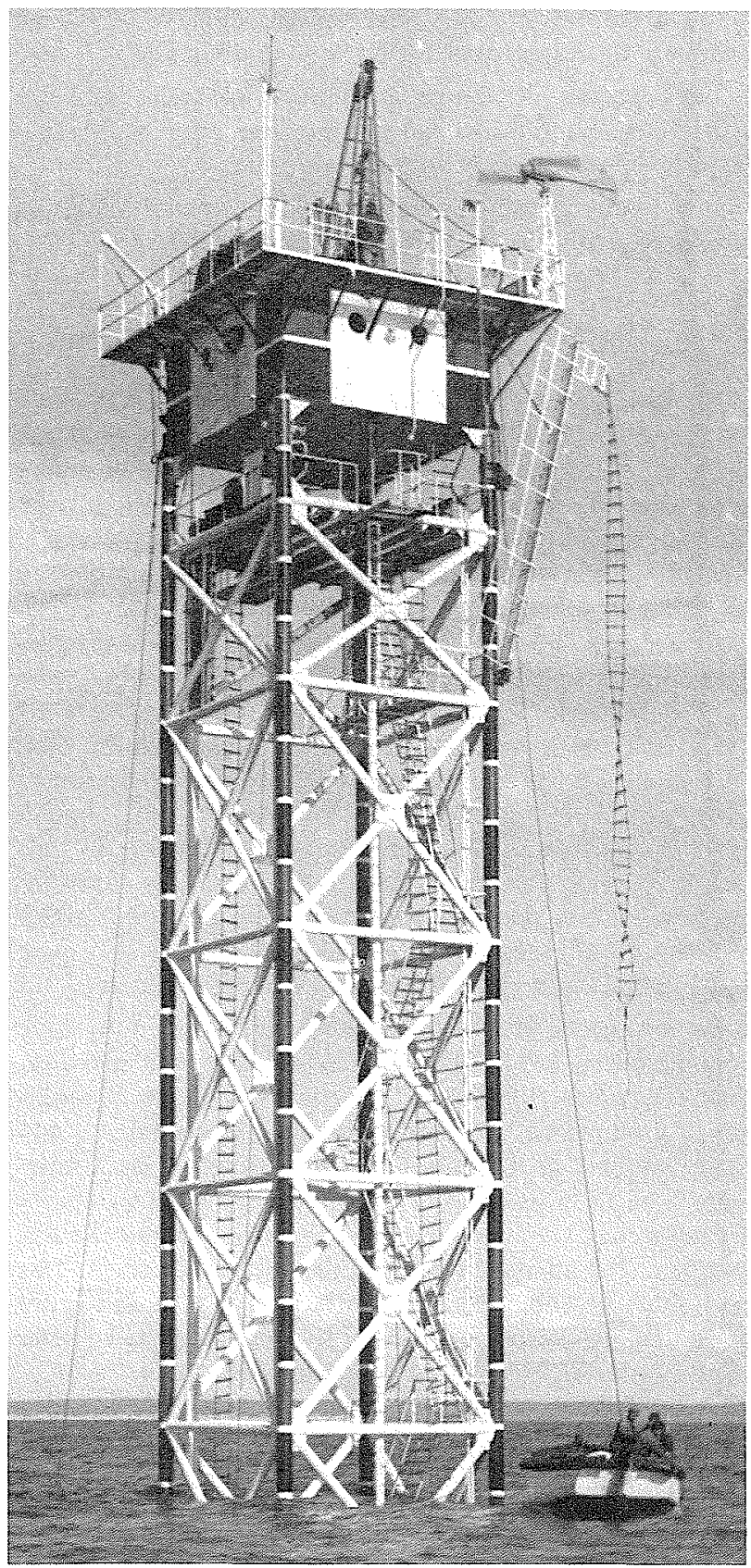

27/ Le Ludion

pables de la maintenir hors d'eau jusqu'au-dessus du fond de $25 \mathrm{~m}$ et comportant 2 derricks de sondages. L'inconvénient de l'engin était de ne pas permettre d'envisager des carottages de plus de $12 \mathrm{~m}$; il effectua cependant, en 1955, 12 sondages sur 12 emplacements différents; l'épaisseur sondée cumulée est $102 \mathrm{~m}$.

De 1958 à 1959 les sondages furent effectués à partir d'une tour de sondage de $40 \mathrm{~m}$ de haut appelée " Ludion» (fig. 27). L'engin comportait un caisson cloisonné (après essais sur modèle réduit au Laboratoire National d'Hydraulique), de façon à ce qu'il puisse s'arracher du fond et venir flotter en surface, ou au contraire se poser sur le fond sans prendre de gîte, en ne commandant la manœuvre que par une seule vanne.
L'engin reposait sur une semelle de 150 tonnes, de diamètre extérieur $17 \mathrm{~m}$, assurant sa stabilité au courant et à la houle. Au cours des trois campagnes de 1957-58-59, 185 carottages furent effectués sur 90 emplacements dans une zone de $10 \mathrm{~km} \times 15 \mathrm{~km}$ au sud de l'archipel des îles Chausey. L'épaisseur totale sondée est $1541 \mathrm{~m}$; le plus long sondage atteint $29 \mathrm{~m}$. Les carottages ont servi à déterminer les épaisseurs de sédiments et de roches altérées, en particulier de tracer la carte du bon rocher (fig. 28) dans la zone de Grouin à Chausey [218].

Des essais de perméabilité des terrains, effectués à la fin des sondages, ont conduit à des débits de 5 à $10 \mathrm{l} / \mathrm{m} / \mathrm{mn}$ pour des pressions de 5 à 9 bars.

Une tour de $25 \mathrm{~m}$ de haut, appelée "Micro-tour», fut utilisée en parallèle pour déterminer les épaisseurs de sédiments par sondage à la lance (fig. 29). Facile d'emploi (fig. 30), elle effectua 1954 sondages de 1957 à 1959.

Le Ludion permit un travail remarquable; mais il est regrettable que souvent les sondages aient dû être interrompus pour des raisons extérieures (courant, houle, époque de relevage,...). Il semble que l'engin était trop léger pour les sondages profonds, et trop lourd pour les sondages de reconnaissance; pour ces derniers, serait idéale la Micro-tour utilisée pour les sondages à la lance.

Bien que la contribution des sondages constituât une importante source de connaissance, elle ne pouvait remplacer l'observation géologique directe. Les sondages furent donc complétés par des prises d'échantillon de roche en place par plongeurs. Deux méthodes furent utilisées pour la reconnaissance des affleurements [10,22]: l'une visuelle par plongeur embarqué sur un engin submersible, dit "aquaplane ", traîné au-dessus du fond par un bâtiment se déplaçant en surface, l'autre au moyen d'un sondeur à ultra-son. Une fois déterminés les emplacements des affleurements, il était procédé à l'étude sur place par des plongeurs initiés au cours d'un stage préliminaire. Sur chaque affleurement le plongeur identifiait une orientation, de préférence celle des couches stratigraphiques et le pendage, et notait l'étendue de l'affleurement et ses caractéristiques. De 1955 à 1959 furent effectuées 2027 plongées qui permirent de rapporter 1433 échantillons, dont 610 avaient un intérêt structural. D'autres échantillons furent récoltés en vue de compléter les recherches géologiques et de mieux cerner le problème de la baie du Mont-Saint-Michel dans son cadre géologique, dont on ne connaissait guère les données dans le domaine maritime. Des reconnaissances géologiques furent effectuées, d'une part, le long de la côte occidentale du Cotentin $[12,14]$, d'autre part aux Minquiers [15].

Toutes les carottes firent l'objet d'une étude géologique au laboratoire, étude résumée sur fichier. Chaque échantillon de plongée étant orienté, il était possible de retrouver au laboratoire les données habituellement recueillies sur les affleurements à terre $[17,23]$.

\subsubsection{Interprétation géologique}

Les cinq campagnes géologiques successives, grâce à l'ensemble des moyens mis en ceuvre, ont fourni une 


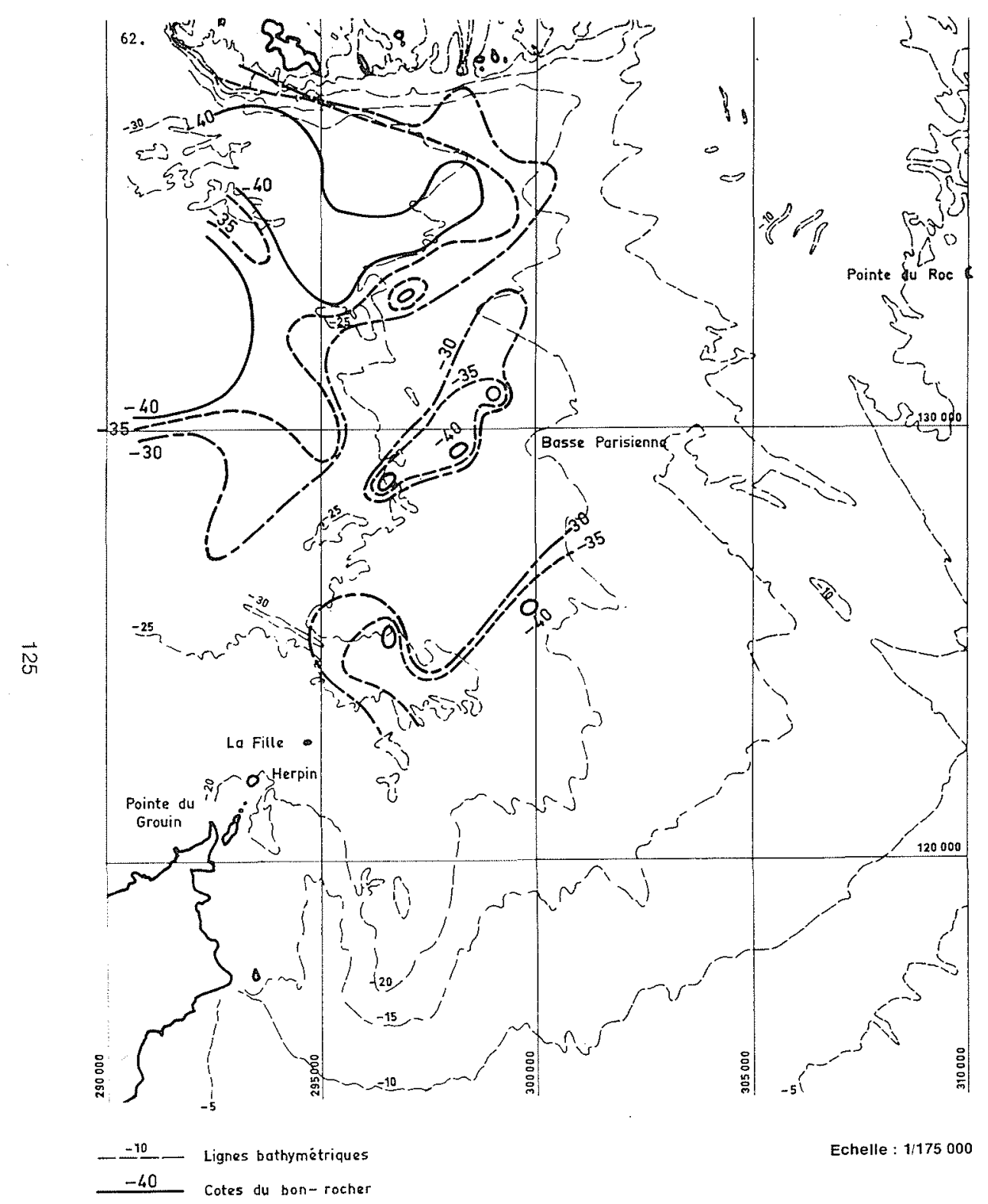

28/ Cote du bon rocher sous le N.G.F.

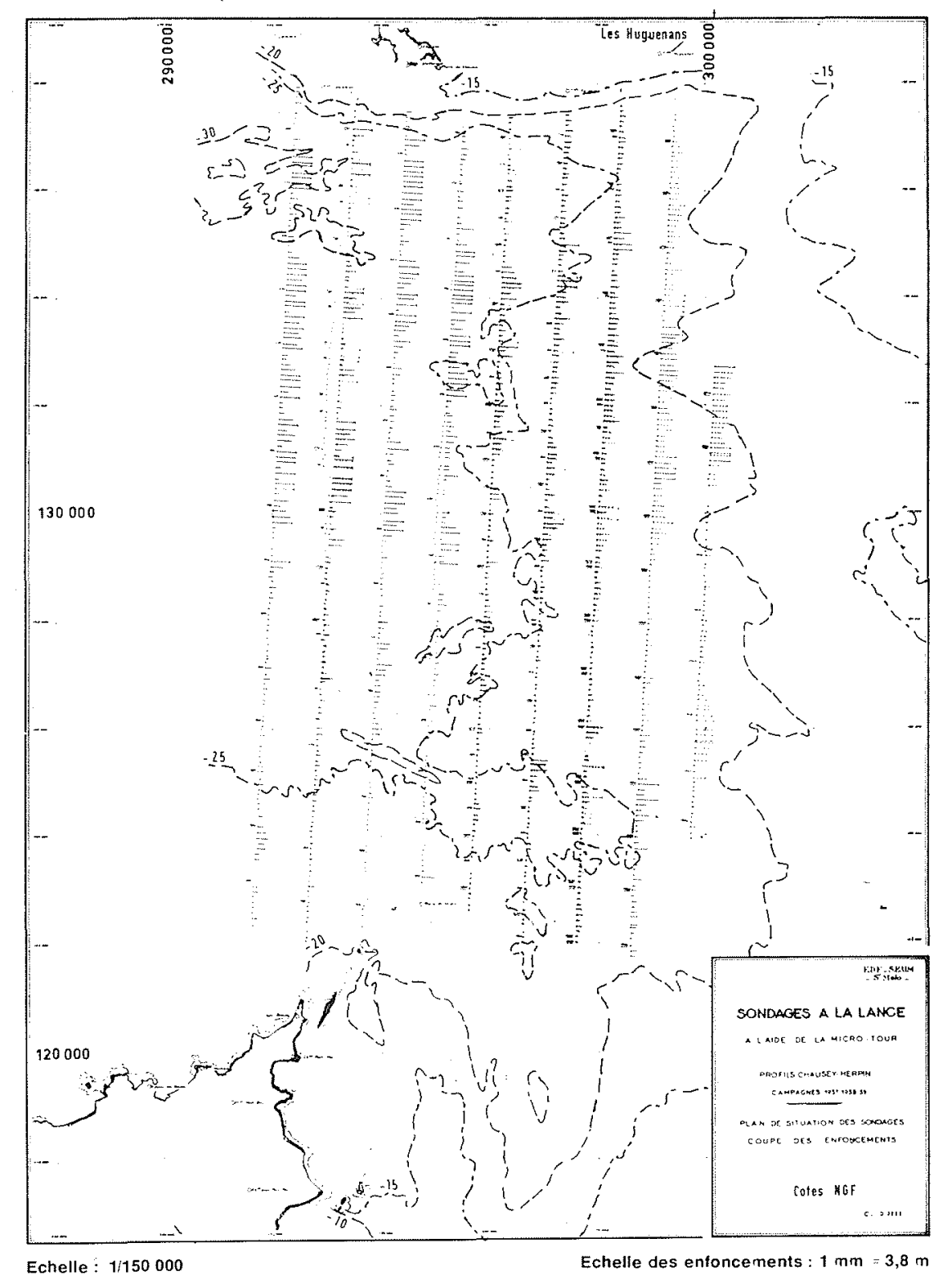

29/ Epaisseur de sédiments d'après les sondages à la lance par la Micro-Tour 


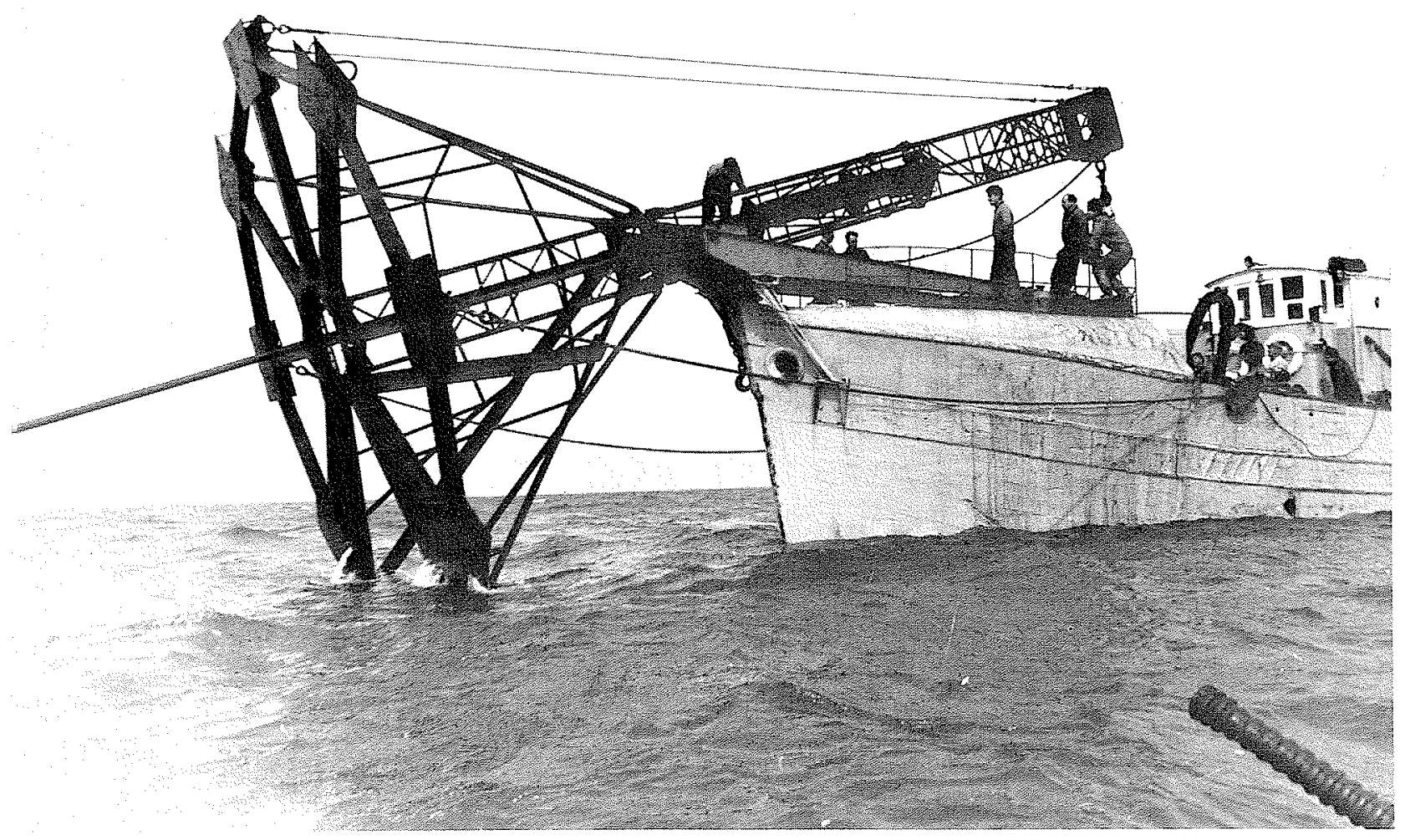

30/ La tour Micro-Ludion

documentation exceptionnelle en qualité et abondance. Elles autorisent donc à proposer l'interprétation géologique fondée, suivante, de M. J. Graindor (fig. 31).

Dans la plus grande partie de la baie du MontSaint-Michel dominent des roches appartenant au Briovérien (du nom latin de Saint-Lô: Briovéra), c’est-àdire au Précambrien supérieur. Ce système stratigraphique, bien connu dans tout l'ensemble de la BasseNormandie, est constitué par des phyllades, des grauwackes et des grès dont la monotonie déroute les noninitiés et rend souvent perplexes les spécialistes.

Dans la périphérie de la baie affleurent trois massifs granitiques: au nord, celui de l'archipel des îles Chausey, à l'est, celui de Carolles qui, en direction de l'est, est prolongé par le granite de Vire, enfin au sud, le granite de Saint-Broladre; ces trois massifs sont antécambriens, mais postérieurs au Briovérien. Enfin, dans la partie S.-W. se trouve, en mer, le prolongement du massif de roches migmatitiques, cristallophylliennes, formant le massif de Saint-Malo. Tout l'ensemble constitue un socle ancien.

L'ensemble de ce matériau ne poserait aucun problème particulier pour les travaux du génie civil, si la très vieille histoire géologique de cette contrée ne s'était accompagnée d'épisodes tectoniques. Ceux-ci ont affecté tout l'ensemble du socle, de manière puissante, à diverses reprises. Il ne s'agit pas de plissements, au sens propre du terme, comme ceux qui ont modelé le
Précambrien local, mais de phénomènes de serrage liés aux compressions entre blocs de la croûte, sollicités par la translation continentale. De ces contraintes mécaniques résultent d'importantes dislocations, chevauchements et cisaillements notamment. Pratiquement, ces phénomènes mécaniques se traduisent par des faisceaux de fractures subordonnés aux dislocations majeures en nombre limité, trois ou quatre au plus, et d'inégale intensité. Ces fractures, allant de la grande faille aux petites diaclases, jouent un rôle géologique important; par exemple, en raison des points faibles qu'elles constituent dans la roche, elles ont déterminé le tracé des cours d'eau, empruntant le trajet le plus facile, ébauché par l'état de fracturation et d'écrasement des roches qui se rencontre dans les failles.

L'étude approfondie, permise par la large mise en œuvre des moyens utilisés pour la prospection géologique, met en évidence la présence de zones fragiles et particulièrement entre Chausey et Cancale (zone mylonitisée, fig. 31) au contact entre deux blocs crustaux de structures différentes: l'une provenant d'une zone profonde, donc très métamorphique, l'autre totalement dépourvue de métamorphisme. La soudaineté du passage dépasse tout ce qui pouvait être prévu; c'est là qu'ont été décelés les profonds enfoncements des sondages à la lance (fig. 29). Le passage entre le bloc de Saint-MaloCancale et le Briovérien de la baie se fait le long d'une grande dislocation de direction N. 120". L'intersection 

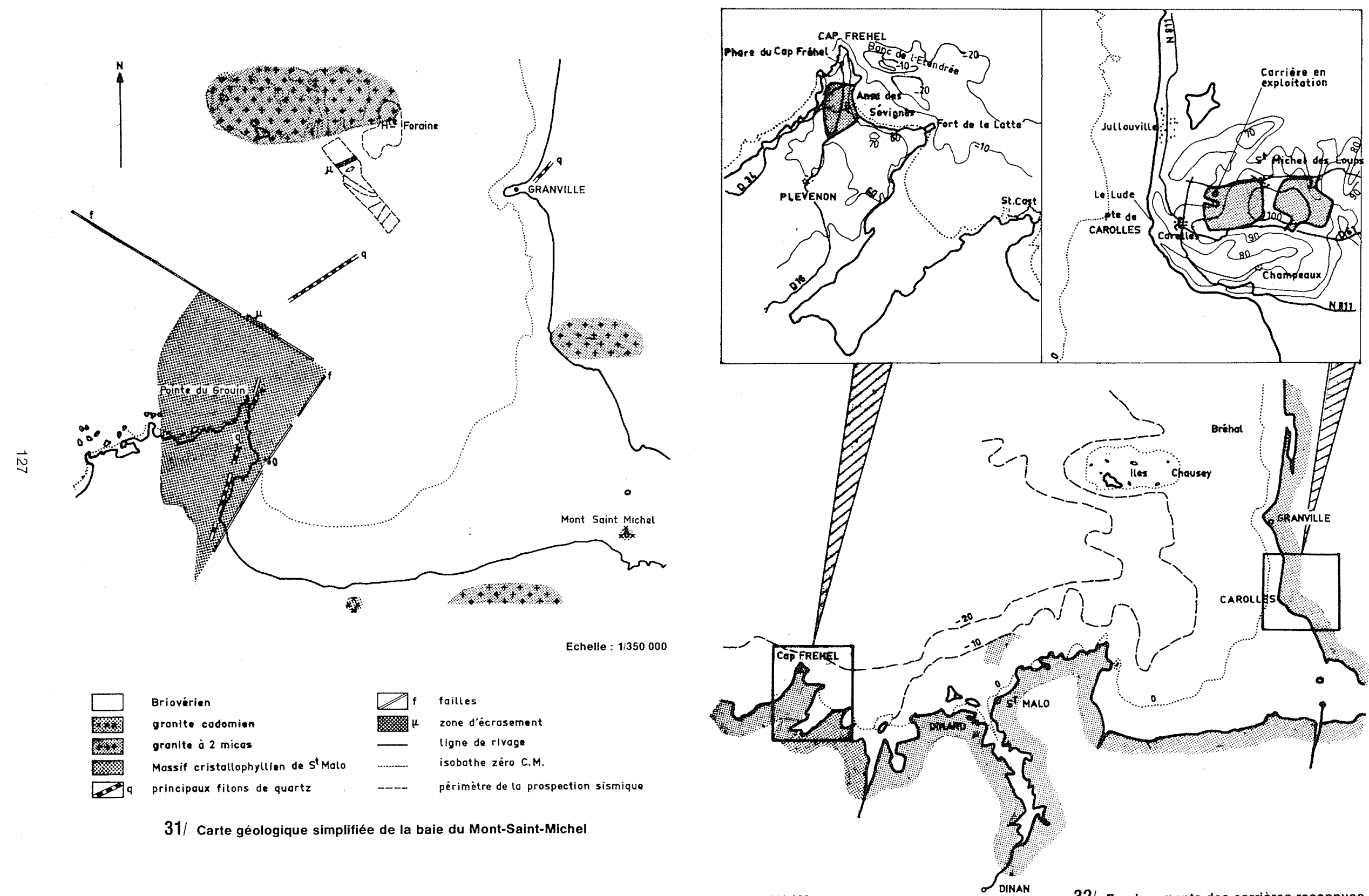

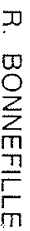




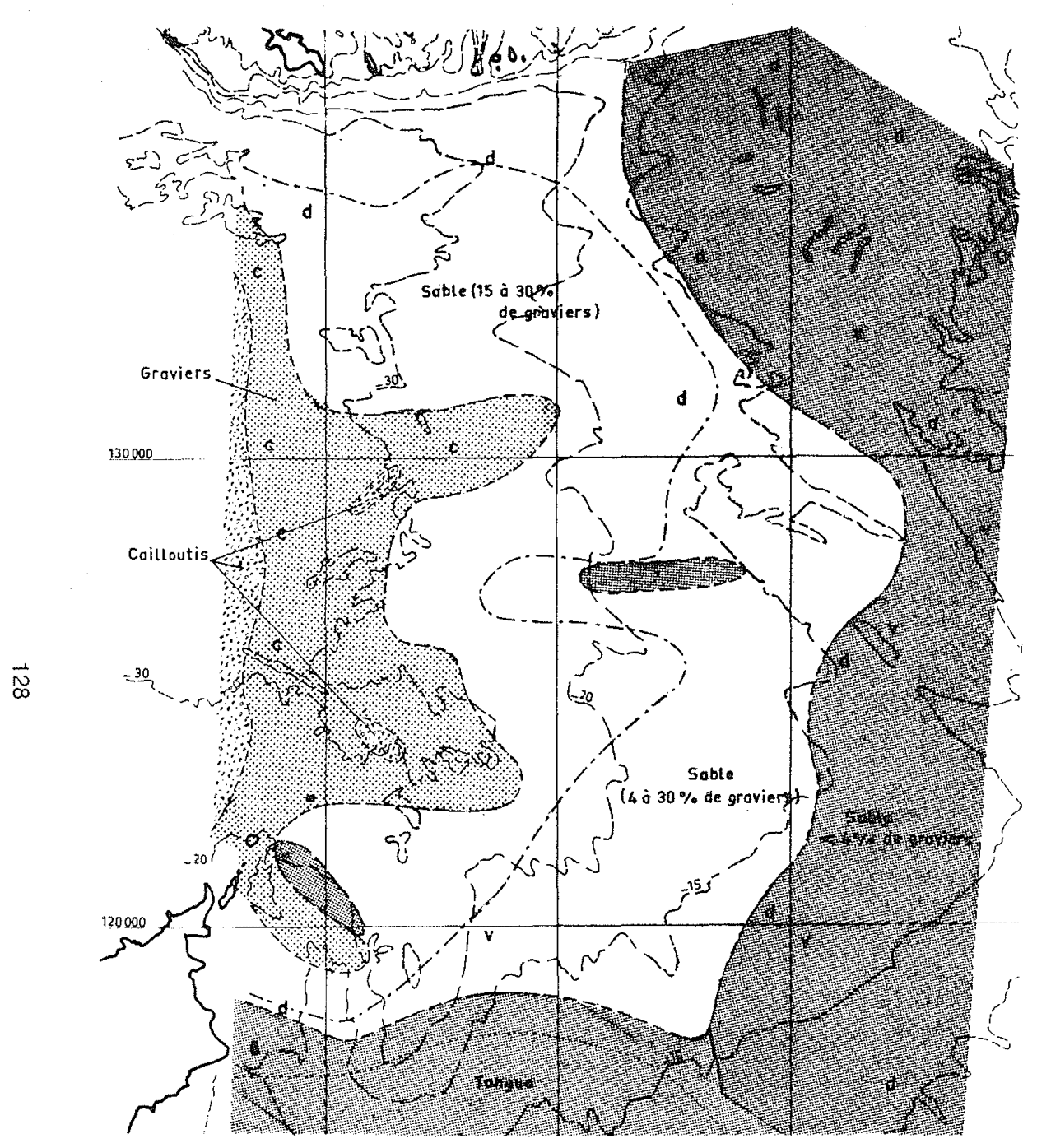

Cotes N.G.F.

33/ Répartition des sédiments dans la baie du Mont-Saint-Michel

$$
c:>25 \% \text { de cailloux } v:>20 \% \text { de vase de débris coquilliers }
$$

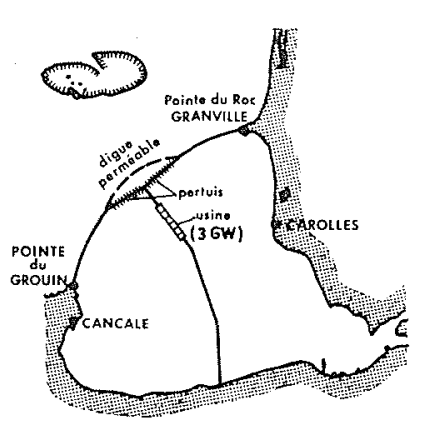

PROJET DE L'ENERGIE DES MAREES 1942

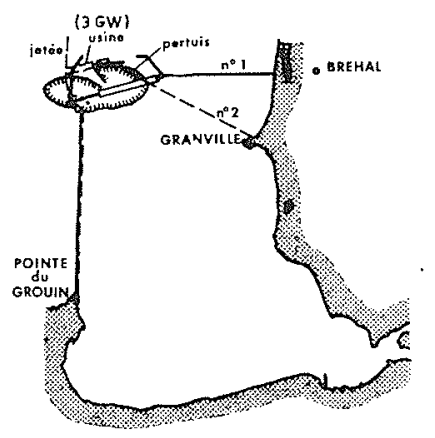

PROJETS J.VELUT 1951 (n 1 at 2)

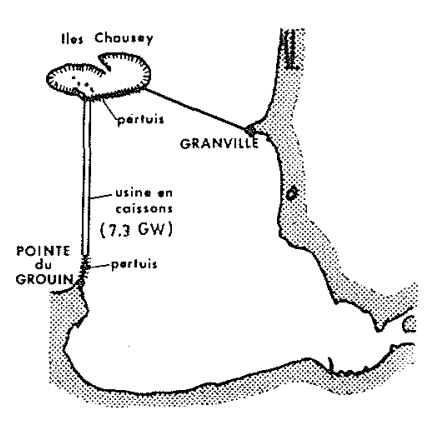

PROJET BOUVIER 1953

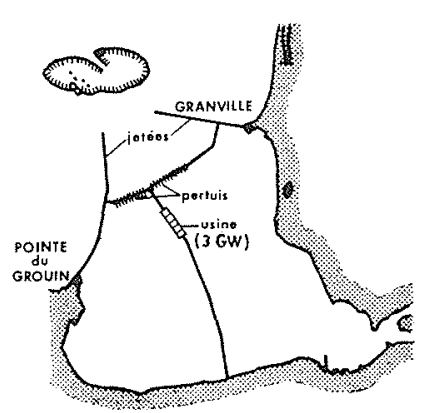

PROJET FLEURY 1947

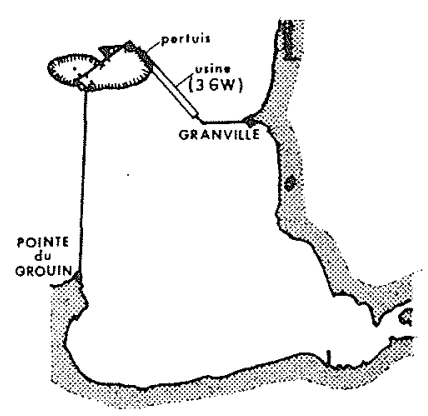

PROJET J.VELUT 1952

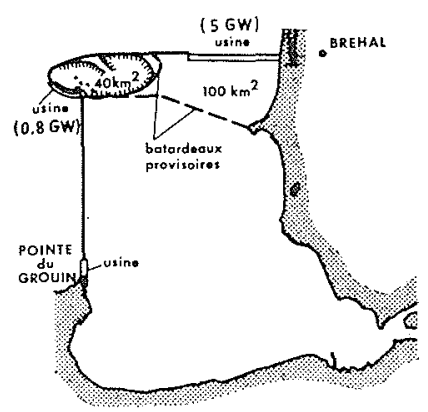

PROJET J.VELUT 1953

34/ Les différents projets de Chausey de 1942 à 1953 
avec la ligne Grouin-Grande lle de Chausey a lieu à $5,2 \mathrm{~km}$ au nord de la pointe du Grouin. Les autres fractures, accompagnées aussi de roches broyées, de filons ou de faisceaux de failles, ne constituent pas des obstacles à l'implantation d'une digue, comparables à celui de l'alignement nord-sud au nord de Cancale.

L'ensemble des observations sur le terrain, le dépouillement des rapports de sondage et des observations des plongeurs, l'étude au laboratoire des carottes et des échantillons et les analyses, fournissent un très volumineux dossier dont les données seront publiées ultérieurement dans un mémoire scientifique détaillé. L'essentiel des conclusions scientifiques se résume comme suit : un ensemble de roches fortement plissées (pendages compris entre $60^{\circ}$ et $90^{\circ}$ ), roches dures appartenant au Précambrien supérieur (antérieur à 600 millions d'années) ayant une direction axiale N. $60^{\circ}$, constitue la majeure partie du substratum de la baie. Le Briovérien de Granville et de Saint-Pair-sur-Mer ou de Saint-Léonard-de-Vain (c'est-à-dire la côte de la baie) donne, sur terre, une bonne image de ce terrain. Au nord, le granite des îles Chausey est en contact mécanique avec le Briovérien, mais le chevauchement ne pose pas le problème du cisaillement signalé au S.-E. de la baie. De nombreuses failles, dont chacun des faisceaux a pour direction dominante, N. $30^{\circ}$, N. $60^{\circ}$, N. $120^{\circ}$, N. $155^{\circ}$, parcourent la région; certaines fractures ont servi à l'intrusion de magma filonien. Enfin, plaqué contre le Briovérien, le bloc crustal cristallophyllien, auquel appartient le massif de Saint-Malo, complète la partie S.-E. de la baie. Les caractéristiques de ce terrain sont bien connues, ce sont celles qui ont été rencontrées dans l'estuaire de la Rance.

\subsubsection{Recherche des carrières}

Une reconnaissance des emplacements possibles fut effectuée par les Carrières de l'Ouest [204]. Quatre sites méritent l'attention (fig. 32) :

- le plateau granitique à l'est de Carolles et au sud de Saint-Michel-des-Loups, pourrait fournir $80 \mathrm{Mm}^{3}$; parmi les solutions d'évacuation, signalons la création d'un port au débouché de la vallée du Lude à $1 \mathrm{~km}$ du site, ou le transport par téléphérique jusqu'à l'enracinement de la digue Nord à $20 \mathrm{~km}$ environ au nord;

- l'archipel de Chausey comporte de nombreuses traces d'anciennes carrières et de petits ports d'embarquement; un gisement possible est situé au nord du Sound:

- un gisement pouvant fournir $50 \mathrm{Mm}^{3}$ a été reconnu au sud de la zone classée du cap Fréhel; un port d'évacuation serait à construire dans l'anse des Se vignes;

- enfin un gisement de grès rose serait exploitable dans les falaises Nord du cap d'Erquy

Il semble que, de tous ces sites, celui de Carolles est le plus réaliste; il pourrait éventuellement suffire pour édifier la digue Nord du projet Chausey. Actuellement, des carrières de granit (pierres tombales et bordures de trottoir) sont ouvertes près de Saint-Michel-des-Loups; en revanche, il serait difficile d'ouvrir une carrière au cap Fréhel.

\subsection{6. Étude sédimentologique}

L'étude sédimentologique de la baie fut confiée à J. Bourcart [26]. Les échantillons furent obtenus par dragage en surface et à partir des carottages [212]. Les sédiments recueillis en surface sont très variés: blocs, cailloux, graviers, sable, coquilles, tangue (poudre calcaire très fine); ils contiennent tous des débris organogènes. Les algues calcaires et les animaux sont abondants; il se forme des masses globulaires, parfois cimentées par les algues calcaires autour d'un gravier qui a servi de support initial. A ces apports organogènes se mêle une importante fraction terrigène d'origine marine.

La fraction biogène (contenant des débris calcaires) se situe au fond de la baie. La fraction terrigène (débris silicieux) se cantonne au large entre Chausey et la pointe du Grouin; elle est aussi plus riche en granules, graviers et cailloux. La taille des apports détritiques augmente vers le large, ce qui prouve leur origine marine, dans les zones où les courants sont forts (fig. 33).

La tangue ( $50 \%$ de poudre calcaire, sablon quartzeux et éléments planctoniques et benthiques) occupe le fond de la baie, où elle est en formation, et les polders et marais en arrière des digues. Sa formation est due à la faible vitesse des courants de flot ne transportant en suspension que les particules fines. Sur la ligne Chausey-Granville $[13,212]$ les argiles, datées du Flandrien moyen, de composition voisine de celle des schistes sous-jacents, se sont déposées en milieu lacustre et ont été peu remaniées par la transgression marine.

La répartition verticale des sédiments montre que la partie terrigène (graviers) s'accroît avec la profondeur; on trouve successivement :

- une couche de dépôts marins calcaires organogène, épaisse de $10 \mathrm{~cm}$ au large et $6 \mathrm{~m}$ au fond de la baie;

- une couche continentale de limons dépourvus d'apports coquillers contenant des cailloux anguleux de nature pétrographique différente de celle du substratum; cette couche est très plate (pente maximale $3 \%)$

- une zone d'altération de la roche sous-jacente d'argile et sablon, c'est-à-dire les constituants des schistes; elle pourrait être due à des fractures qui ont broyé les schistes; son épaisseur peut atteindre $40 \mathrm{~m}$.

Signalons enfin la thèse de J. Fortin [42], qui a établi une carte des dépôts meubles du plateau des îles Chausey et précisé l'origine, dans le temps et l'espace, du matériel sédimentaire de cet archipel. A l'ouest dominent les dépôts terrigènes; à l'est les courants de marée ont édifié des bancs de sables coquilliers. La rencontre des courants provenant du nord et du sud transforme la partie orientale en un piège hydraulique efficace, alimenté à partir des fonds sous-marins environnants.

\subsubsection{Conclusion}

En résumé, la seule difficulté majeure du point de vue géologique semble se localiser sur le tracé nord-sud de l'usine. Pour l'éviter, dans le cas où les arguments géologiques auraient un poids suffisant dans la définition du projet, il faudrait adopter pour la digue-usine un tracé courbe à convexité vers l'ouest, quitte à y prolonger la 


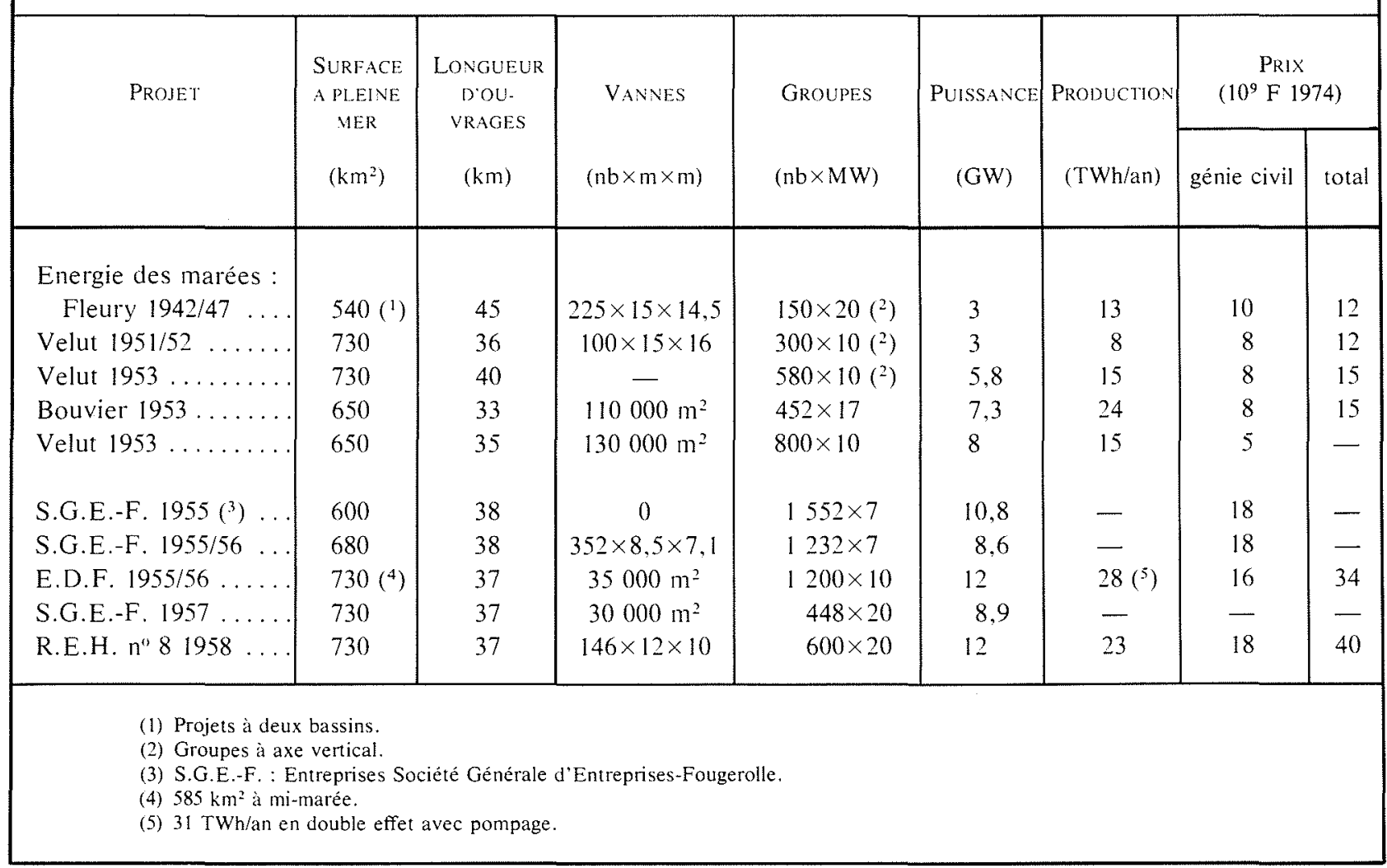

prospection géologique. La zone difficile, au voisinage de la pointe du Grouin, étant traversée par une digue de fermeture, ne poserait qu'un problème secondaire de génie civil.

Il convient enfin de souligner que, du fait de ces études, la géologie a bénéficié de premières informations qui enrichissent nos connaissances, et que rarement un problème de ce genre n'a reçu plus amples moyens d'investigation avec autant de continuité.

\subsection{L'historique des avant-projets de l'usine de Chausey}

\subsubsection{Les projets antérieurs à 1950}

\subsubsection{LE PROJET DE 1942 ( $3 \mathrm{GW}$ )}

Le premier avant-projet détaillé fut établi par la Société «L'Energie des Marées " en 1942 [201]. C'est un projet à deux bassins (fig. 34), limité côté mer par une digue semi-circulaire entre la pointe du Grouin et la pointe du Roc. L'usine est située dans la digue intermédiaire isolant deux bassins de surfaces sensiblement égales (bassin supérieur côté Carolles $295 \mathrm{~km}^{2}$, bassin inférieur côté Cancale $245 \mathrm{~km}^{2}$ ). Les pertuis de vannage sont protégés contre la houle par une digue perméable de $3,2 \mathrm{~km}$ de long constituée de piliers elliptiques $(25 \times 10 \mathrm{~m})$, espacés de $22 \mathrm{~m}$, débitant $187000 \mathrm{~m}^{3} / \mathrm{s}$. Les digues de clôture $(6,5 \mathrm{~km}$ à l'est, $8 \mathrm{~km}$ à l'ouest) et intermédiaires $(18,3 \mathrm{~km})$ sont en enrochements. Les ca- ractéristiques du projet sont résumées dans le tableau 8 pour faciliter la comparaison avec les projets ultérieurs.

L'usine était équipée de 150 groupes de $20 \mathrm{MW}$, à axe vertical, de diamètre $8 \mathrm{~m}$ et de débit $334 \mathrm{~m}^{3} / \mathrm{s}$ $\left(50000 \mathrm{~m}^{3} / \mathrm{s}\right.$ au total)

La production serait, dans les trois hypothèses suivantes:

- fonctionnement en recherchant la production maximale, c'est-à-dire sans limitation artificielle: 12,8 TWh/an ;

- fonctionnement en recherchant la production continue, c'est-à-dire marche sans arrêt : 10,8 TWh/an;

- équipement réduit à $1 / 3$, pendant les phases de travaux : 6,4 TWh/an.

Les points faibles du projet sont la trop grande concentration des vannes, les pertes de charge dans la digue perméable et l'usilisation des groupes à axe vertical. Le rapport $E / L$, entre l'énergie naturelle (66 $000 \mathrm{GWh} / \mathrm{an})$ et la longueur totale des ouvrages $(45 \mathrm{~km})$, vaut $1,45 \mathrm{GWh} / \mathrm{m}$.an; il est faible. En conclusion le projet, sous-équipé. traduit la prudence des pionniers; il est intéressant de rappeler que les auteurs du projet proposaient de construire au préalable une usine «maquette» de $2 \mathrm{MW}$ dans l'anse de Primel (baie de Morlaix).

Le projet fut repris en 1954 [210], en le supposant équipé de groupes bulbes type Rance, ce qui permit de conclure à la possibilité d'une production de 14 TWh/an pour le prix de 23 milliards de francs 1939, se décomposant en : 
- digue étanche ..... 5 milliards de francs 1939

- digue de protection.. 3

- vannes.......... 5

- digue usine ....... 6

- équipement ....... 4

\subsubsection{Le PROJET « FLeURY » 1947}

Le tracé des ouvrages est sensiblement le même que celui du projet 1942, mais les pertuis de vannage sont protégés par des jetées délimitant un avant-port ouvert au N.-W., face à l'archipel de Chausey, jouant le rôle de brise-lame (fig. 34). Le projet est beaucoup plus sérieux du point de vue travaux maritimes; il comporte en outre une évaluation des dépenses de génie civil : 20 milliards de francs 1939 (10 milliards de francs 1974) dont 4 milliards pour les $27.10^{6} \mathrm{~m}^{3}$ de matériaux des digues d'enclôture [150 F/m ${ }^{3}$ (1939)].

Les projets de 1942 et 1947 ont été réestimés de 1945 à 1947. L'étude des prix faite par Miche est la plus intéressante; basée sur l'expérience des travaux maritimes de l'époque, elle tient compte de la longueur du transport $(60 \mathrm{~km})$ et des conditions de navigation très sévères du site. Les estimations en francs 1939 varient de 50 à $250 \mathrm{~F}$ le $\mathrm{m}^{3}$ moyen de digue; une moyenne de $150 \mathrm{~F} / \mathrm{m}^{3}$ (1939), soit $75 \mathrm{~F} / \mathrm{m}^{3}$ (1974), est à retenir.

\subsubsection{Les projets de 1950 à 1955 [210]}

A cette époque, la Commission d'Etudes, dirigée par le S.E.U.M., aborda les problèmes concrets et ouvrit le débat entre les modes de construction entre batardeaux, en usine souterraine ou par échouage de caissons préfabriqués.

\subsubsection{LES PROJETS « J. VelUT » ( 3 ET $5,8 \mathrm{GW})$}

Quatre études successives furent présentées de 1951 à 1953 (fig. 34) :

\subsection{Les deux projets 1951 (usine sur l'archipel de Chausey)}

L'idée directrice est de construire à sec, sur le plateau de Chausey, l'usine et les pertuis, à l'abri de batardeaux édifiés aux limites de l'archipel; les terrassements $\left(58.10^{6} \mathrm{~m}^{3}\right)$ à effectuer pour les ouvrages serviraient à construire la moitié des digues d'enclôture, du bassin $\left(70.10^{6} \mathrm{~m}^{3}\right)$ et les jetées de protection contre la houle $\left(12.10^{6} \mathrm{~m}^{3}\right)$.

L'usine de 300 groupes à axe vertical de $10 \mathrm{MW}$ fonctionnerait à simple effet et produirait $8 \mathrm{TWh} / \mathrm{an}$.

\subsection{Le projet 1952 (usine face au Cotentin)}

Pour supprimer les jetées de protection contre la houle du large, l'usine est ouverte face à la côte du Cotentin. Les ouvrages en béton sont construits à sec à l'abri de batardeaux $\left(12.10^{6} \mathrm{~m}^{3}\right)$. Les trois digues d'enclôture ont les caractéristiques suivantes:

$\begin{array}{lrr}\text { - Nord (sur Chausey) ....... } & 6 \mathrm{~km}, & 4.10^{6} \mathrm{~m}^{3} \\ \text { - Ouest (Chausey-Grouin) } \ldots \ldots & 18 \mathrm{~km}, & 48.10^{6} \mathrm{~m}^{3} \\ \text { - Est (Granville) ............ } & 6 \mathrm{~km}, & 7.10^{6} \mathrm{~m}^{3} .\end{array}$

En plus des fouilles et le réemploi des matériaux des batardeaux, les carrières devraient fournir $50.10^{6} \mathrm{~m}^{3}$.
Les principales objections à faire à cette disposition sont les mauvaises conditions d'alimentation de l'usine dans une zone de faible profondeur. Aux trois projets J. Velut il a été, de plus, objecté les inconvénients de concentrer les travaux sur la zone exiguë de Chausey, et de construire des batardeaux dans des régions très exposées à la houle.

\subsection{Le projet 1953 (usine face au nord)}

Pour augmenter la puissance installée, une usine de 500 groupes de $10 \mathrm{MW}$ est installée face au nord, dans une zone de faible profondeur ( $-5 \mathrm{~m} \mathrm{CM}$ ); ceci facilite la construction dans une enceinte batardée, mais nuit à l'alimentation de l'usine.

Une petite usine de 80 groupes est installée sur la bordure S.-W. de l'archipel. Il est prévu que 12 de ces groupes soient mis en service au bout de 5 ans, puis 50 au bout de 8 ans en utilisant un bassin de $40 \mathrm{~km}^{2}$ ceinturé par des digues autour de l'archipel, bassin étendu à $100 \mathrm{~km}^{2}$ pour les 80 groupes. Le batardeau nord de ce bassin serait progressivement remplacé par les caissons usines échoués, dans des conditions idéales, à partir de la côte. Au bout de la treizième année, 150 groupes seraient en service; parallèlement serait construite la digue Chausey-Grouin.

Ce projet a l'intérêt de montrer les complications entraînées par le souci de produire rapidement de l'énergie pour réduire le poids des intérêts intercalaires. Ceci suggère qu'il vaudrait mieux construire "vite " un projet simple, ce qui revient à dire qu'un effort intensif et bien préparé serait un gage de succès des usines marémotrices.

\subsubsection{Le Projet «Bouvier » OU SGE-Fougerolle 1954} $(7,3 \mathrm{GW})$

Ayant mis en évidence les difficultés rédhibitoires de l'usine entre Chausey et Granville, le projeteur propose (1953-54) une usine composée de caissons échoués entre la pointe du Grouin et Chausey, bordée par des caissons-pertuis, et de Chausey à Granville une digue morte en enrochements ou en sable (fig. 34). Les groupes bulbes apparaissent pour la première fois.

Il était prévu 597 caissons, soit :

- 531 caissons répartis en 9 usines, chacune comportant 48 groupes bulbes de $17 \mathrm{MW}$ et 11 caissons divers (ateliers, transformateur, etc.) comportant en outre chacun $150 \mathrm{~m}^{2}$ de pertuis $\left(88000 \mathrm{~m}^{2}\right)$;

- 66 caissons vannes $\left(22000 \mathrm{~m}^{2}\right)$ de caractéristiques suivantes: longueur $52 \mathrm{~m}$, largeur $26 \mathrm{~m}$, hauteur $38 \mathrm{~m}$, tirant d'eau $23 \mathrm{~m}$, masse 32000 tonnes.

\subsubsection{L'USINE SOUTERRAINE (3 GW)}

L'usine souterraine proposée par J. Velut, en 1950, comportait des groupes à axe vertical dont une particularité était la possibilité d'établir une communication entre la bâche et l'aspirateur, susceptible de remplacer les pertuis de vannage, le tout étant disposé dans un tunnel sous la digue. Le projeteur proposait d'installer 150 groupes de $20 \mathrm{MW}$, distants de $150 \mathrm{~m}$ sous la digue entre Chausey et Granville. Le projet avait l'avantage de bien répartir les groupes, de permettre l'équipement 
progressif de l'usine; mais les problèmes de creusement et de maintien des tunnels ne furent pas évoqués.

Une variante fut proposée par J. Velut en 195I; la turbine était disposée au fond d'un caisson-batardeau, d'abord circulaire, puis rectangulaire $(40 \mathrm{~m} \times 20 \mathrm{~m})$ à - $37 \mathrm{~m} \mathrm{CM}$, alimenté par un aspirateur tronconique. Tout un système de disposition des caissons permettait de réduire les actions de la houle.

\subsubsection{Projet « J. Velut » de 1953 (8 GW)}

Le projet est une extrapolation du projet Rance dans le rapport 30 ; il est très voisin du projet Bouvier, dont il ne diffère que par les dimensions des caissons et les dispositions des vannes. Pour retrouver une symétrie de l'écoulement par les groupes bulbes, ceux-ci sont disposés tête-bêche; ils offrent une section de $50000 \mathrm{~m}^{2}$ de pertuis, et sont calés très bas, donc protégés de la houle. Le vannage complémentaire est obtenu par $80000 \mathrm{~m}^{2}$ de pertuis dans l'archipel de Chausey, donc protégé de la houle.

Les caissons de ce projet ont un faible tirant d'eau $(17 \mathrm{~m})$ et ne comportent que $5000 \mathrm{~m}^{3}$ de béton. Les 800 caissons prévus seraient échoués en dix ans; les pertuis dans l'archipel et la digue Chausey-Granville seraient construits en trois ou quatre ans. Les estimations de prix sont $240.10^{6} \mathrm{~F} 1953$ par caisson $\left(6.10^{6} \mathrm{~F} 1974\right)$, soit 4,8 milliards de francs 1974 pour 800 caissons.

J. Velut a proposé en outre de construire les digues mortes en injectant du sable sous un tapis large de $120 \mathrm{~m}$ en "lucoflex", disposé au préalable sur le fond et lesté à ses extrémités. Il faudrait six ou sept ans pour remblayer les $50.10^{6} \mathrm{~m}^{3}$ de sable nécessaires, pour un prix de l'ordre de 15 milliards de francs 1951 .

\subsubsection{Réflexions sur les projets antérieurs à 1955}

Les projets ont un point commun : la fermeture de la baie du Mont-Saint-Michel par un ensemble d'ouvrages de 30 à $40 \mathrm{~km}$ environ délimitant un bassin de 500 à $700 \mathrm{~km}^{2}$. Les ouvrages de génie civil, usines et pertuis, n'occupent qu'une partie des ouvrages d'enclôture.

L'historique des projets traduit le passage des machines à axe vertical aux groupes bubles. Les groupes bulbes apportent un accroissement de la puissance installée par mètre linéaire de digue, donc une diminution du volume de béton à mettre en cuvre; de plus ils permettent les cycles à double effet, L'expérience de la Rance confirme leur intérêt.

L'historique des projets traduit l'évolution des digues en enrochements vers les ouvrages préfabriqués en béton. Cette dernière technique offre des avantages certains : économie, préfabrication, utilisation du béton, ce qui reporte loin du site la détérioration des paysages par les carrières. Mais ce procédé, en plein essor à l'époque où le prix du fuel diminuait, donc parallèlement le prix du béton gros consommateur de pétrole, sera-t-il encore le seul valable à la fin de ce siècle. Il faudrait actuellement tenir compte des progrès réalisés dans la mise en œuvre des grandes digues en sable recouvertes d'une protection légère, technique qui n'était pas courante il y a vingt ans, sans oublier l'utilisation possible des tétrapodes dans les zones particulièrement exposées.
Les premiers projeteurs avaient le souci constant de défendre leurs ouvrages contre la houle; peut-être avaient-ils une meilleure connaissance des problèmes maritimes que leurs successeurs qui, ensuite, ont plus ou moins ignoré ce problème. L'économie de leur projet s'en ressent; en particulier le mauvais choix, confirmé par les essais sur modèle réduit, de la ligne ChauseyGranville pour implanter l'usine, condamne beaucoup ces projets. Les essais en canal à SOGREAH et sur microréseau à E.D.F. ont montré que la crainte de la houle était exagérée; mais ce sujet n’a pas encore été assez approfondi. On se rappelle les incidents lors de la construction de la Rance dus à une sorte de scepticisme vis-à-vis des effets de la houle sur le site. Compte tenu du progrès réalisé depuis 1950 dans la technique antihoule (caisson Jarlan, anti-franchissement, etc.) le problème serait actuellement facilement résolu.

Le prix élevé des ouvrages montre que la validité du projet est essentiellement liée à la rapidité d'exécution du génie civil. Il y a intérêt à produire rapidement un peu d'énergie, quitte à ce que l'équipement hydroélectrique soit ensuite progressif. C'est donc en se basant sur ce critère que doit être choisi le type d'ouvrage: par exemple la progression de digues en sable ou en enrochements est peu perturbée par le mauvais temps, sauf les tempêtes; en revanche, la mise en place des caissons exige un certain calme. L'utilisation des caissons peut d'ailleurs être un sujet à soumettre à l'imagination des constructeurs. En effet les caissons doivent satisfaire à des exigences contradictoires:

- tirant d'eau minimal ou conduisant au minimum de travaux à exécuter en mer;

- ouverture maximale entre la mer et le bassin;

- liaison étanche entre eux et avec le fond;

- protection contre la houle.

Les premiers projets ont aussi mis en relief l'absurdité de construire l'usine sur les hauts fonds, par exemple sur l'archipel de Chausey, par suite des énormes déroctages que cela entraînerait. L'idée en a germé qu'il est préférable de choisir les talwegs comme tracé des usines marémotrices; L. Vantroys l'a concrétisé sous forme de deux tracés :

- pointe du Grouin-Granville, déjà proposé en 1942, long de $25 \mathrm{~km}$, et isolant un bassin de $300 \mathrm{~km}^{2}$ à mi-marée ;

- cap Fréhel-pointe d'Agon, long de $65 \mathrm{~km}$, isolant un bassin de $1300 \mathrm{~km}^{2}$ à mi-marée, et passant par des fonds de l'ordre de 20 à $25 \mathrm{~m} \mathrm{CM}$.

Finalement, il reste à se demander si l'utilisation simultanée des digues en sable et des caissons n'est pas une solution valable comme le suggérait $J$. Velut en 1956. La méthode pourrait être la suivante :

- réalisation de digues en sable à l'avancement, ou plus précisément en boudins de sable, matériau abondant localement et provenant des fouilles de l'usine; ces digues en sable seraient des ouvrages définitifs pour la digue Nord, mais provisoires pour la digue Ouest, cette dernière construite parallèlement à l'usine et un peu au large;

- échouage des caissons usine-pertuis derrière la digue en sable à l'avancement;

- dès le bassin totalement fermé par les digues en sable, mise en service de la première portion d'usine, 
après démontage de la digue en sable située au large :

- continuation de l'avancement de l'usine en caissons, puis mise en route de la deuxième portion d'usine, etc.

Les raccordements usine-digue en sable seraient préparés sous forme de batardeaux en palplanches perpendiculaires à l'axe de l'usine mis en place avant l'édification de la digue en sable.

\subsubsection{Les projets postérieurs à 1955}

La période 1953-55 est une phase de réflexions sur les différents modes de construction, assorties d'estimations diverses qui seront reprises plus loin. Des projets plus concrets et plus détaillés sont préparés; tous concernent une usine équipée de groupes bulbes entre la pointe du Grouin et Chausey et un seul bassin, l'usine fonctionnant à double effet.

\subsubsection{LE PROJET S.G.E.-Fougerolle 1955 (10,8 GW)}

II s'agit d'une variante du projet Bouvier, équipé de 1552 groupes de $7 \mathrm{MW}$ type Rance de l'époque (diamètre $5,65 \mathrm{~m})$, comportant :

- Entre Grouin et Chausey :

336 caissons à 4 groupes par fonds de $-20 \mathrm{~m} \mathrm{CM}$, 104 caissons à 2 groupes par fonds de $-15 \mathrm{~m} \mathrm{CM}$, 56 caissons divers:

- Entre Chausey et Granville, une digue en enrochements et sable.

Il n'y a pas de vannes.

Le planning de travaux prévoit la mise en place des 496 caissons en dix ans et de l'ensemble du génie civil en quatorze à dix-huit ans. Aucune étude d'optimisation de ce projet n'a été entreprise, ni d'étude de sa production. Les estimations de dépenses de génie civil sont les suivantes, en francs 1956 :

- une digue-usine $(13,1 \mathrm{~km}) 1,25.10^{6} \mathrm{~m}^{3}$ de béton, $655.10^{9} \mathrm{~F}$ soit $50 \mathrm{MF} / \mathrm{m}[1,2 \mathrm{MF} / \mathrm{m}(1974)]$;

- digues mortes $(24,6 \mathrm{~km}) ; 113.10^{9} \mathrm{~F}$ soit $5 \mathrm{MF} / \mathrm{m}$ [120 000 F/m (1974)];

— total génie civil : $769.10^{9} \mathrm{~F}\left(8.10^{9} \mathrm{~F} 1974\right)$.

\subsubsection{Le projet S.G.E.-Fougerol.te $1955 / 56(8,6 \mathrm{GW})$}

Les entreprises présentèrent, en juin 1956 [213b], un dossier détaillé sur un avant-projet dénommé 1955/56. comportant 1232 groupes de $7 \mathrm{MW}$ type Rance, répartis en 11 usines de 112 groupes superposés comme dans le projet précédent (fig. 35 ).

Les 429 caissons de $57 \times 29 \times 39 \mathrm{~m}$, tirant d'eau $23 \mathrm{~m}, 33900$ tonnes, se répartissent en :

$-28 \times 11=308$ caissons quadrigroupes;

- $2 \times 11=22$ caissons transformateurs;

- $2 \times 11=22$ caissons bi-vannes $\left(4400 \mathrm{~m}^{2}\right.$ au total);

- 77 caissons quadrivannes $\left(30800 \mathrm{~m}^{2}\right.$ au total).

Le projet comporte $35200 \mathrm{~m}^{2}$ de pertuis; compte tenu des groupes ( $37 \mathrm{~m}^{2}$ chacun), la surface de vannage est $80300 \mathrm{~m}^{2}$.

Par rapport au projet 1955 , le tracé de la digue Nord est différent; il rejoint la côte du Cotentin aux Roches de Bréhal. Il est prévu d'utiliser les carrières du cap Fréhel. L'exécution durerait quinze ans et coûterait $770.10^{9} \mathrm{~F} 1956$ répartis en :

— installation, terrassements et divers ... $145.10^{9} \mathrm{~F}$

- digues et palplanches ............ $90.10^{9} \mathrm{~F}$

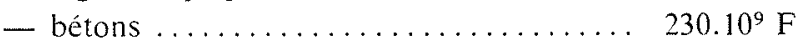

- aciers .................... 305.10 $0^{9} \mathrm{~F}$

\subsubsection{LE PROJET E.D.F. $1955 / 56$ [210]}

A la fin de 1955, le S.E.U.M. souhaitant chiffrer un avant-projet concret techniquement réalisable, ce Service étudia neuf équipements différents. Les groupes sont du type Rance $(5,65 \mathrm{~m}, 88,2 \mathrm{tr} / \mathrm{mn}, 10 \mathrm{MW})$; les neuf équipements sont les combinaisons des choix suivants :

- nombre de groupes : 1 000, 1552 (S.G.E.-Fougerolle 1955) ou 2000 ;

- surface de vannage : $0,35000 \mathrm{~m}^{2}$ ou $70000 \mathrm{~m}^{2}$

La surface de vannage, $35000 \mathrm{~m}^{2}$, est obtenue en multipliant celle relative à la Rance $\left(1000 \mathrm{~m}^{2}\right)$ par le rapport des surfaces des bassins (35) des usines de Chausey et de la Rance.

La stratégie d'exploitation est la recherche du maximum d'énergie avec des cycles à double effet sans pompage. Les estimations sont basées sur les prix unitaires suivants (1956) :

— caissons ......... 30 à $40 \mathrm{MF} / \mathrm{m}$,

— digues ......... 6 à $12 \mathrm{MF} / \mathrm{m}$ suivant la profondeur,

- électromécanique ... prix du projet Rance.

Dans ces conditions, les valeurs optimales sont 1200 groupes de $10 \mathrm{MW}$ et $35000 \mathrm{~m}^{2}$ de vannes, donc très proches de celles du projet S.G.E.-Fougerolle 1955/56. La production annuelle est $27,5 \mathrm{TWh} / \mathrm{an}$ sans pompage et $31 \mathrm{TWh} / \mathrm{an}$ avec pompage; en se basant sur $16 \mathrm{~km}$ de caissons, $21 \mathrm{~km}$ de digues, 50 fois la Rance, le prix serait de $34.10^{9} \mathrm{~F} 1974$.

\subsubsection{L'USINE SOUTERraINe ( $12 \mathrm{GW})$}

Un avant-projet d'usine souterraine fut établi en janvier 1957 [213c]. Il s'agit d'un tunnel de $16 \mathrm{~km}$ de long comportant 600 groupes de $20 \mathrm{MW}$ distants de $25 \mathrm{~m}$, conduisant à effectuer $52.10^{6} \mathrm{~m}^{3}$ de déblais en souterrain et $13.10^{6} \mathrm{~m}^{3}$ de béton en souterrain, disposés entre la pointe du Grouin et Chausey. Les digues de fermeture suivent le tracé du projet E.D.F. 1955/56. Il est à noter que les déblais souterrains suffiraient pour confectionner les agrégats du béton, et pourraient fournir $70 \%$ des matériaux constructifs des digues; le reste, $34.10^{6}$ tonnes, proviendraient des carrières du cap Fréhel.

Le projet a été évalué à 1.5 milliard de F 1957 pour le génie civil; mais la nature géologique du sous-sol condamne ce projet.

\subsubsection{L'AVAVT.. projet par CaISSONS} S.G.E.-Fougeroli.e 1957 (8, 9 et $10,6 \mathrm{GW})$

\subsection{Projet de février 1957 [213d]}

Les études du projet E.D.F. $1955 / 56$ furent reprises en 1957 en utilisant des groupes de $20 \mathrm{MW}$ (diamètre 


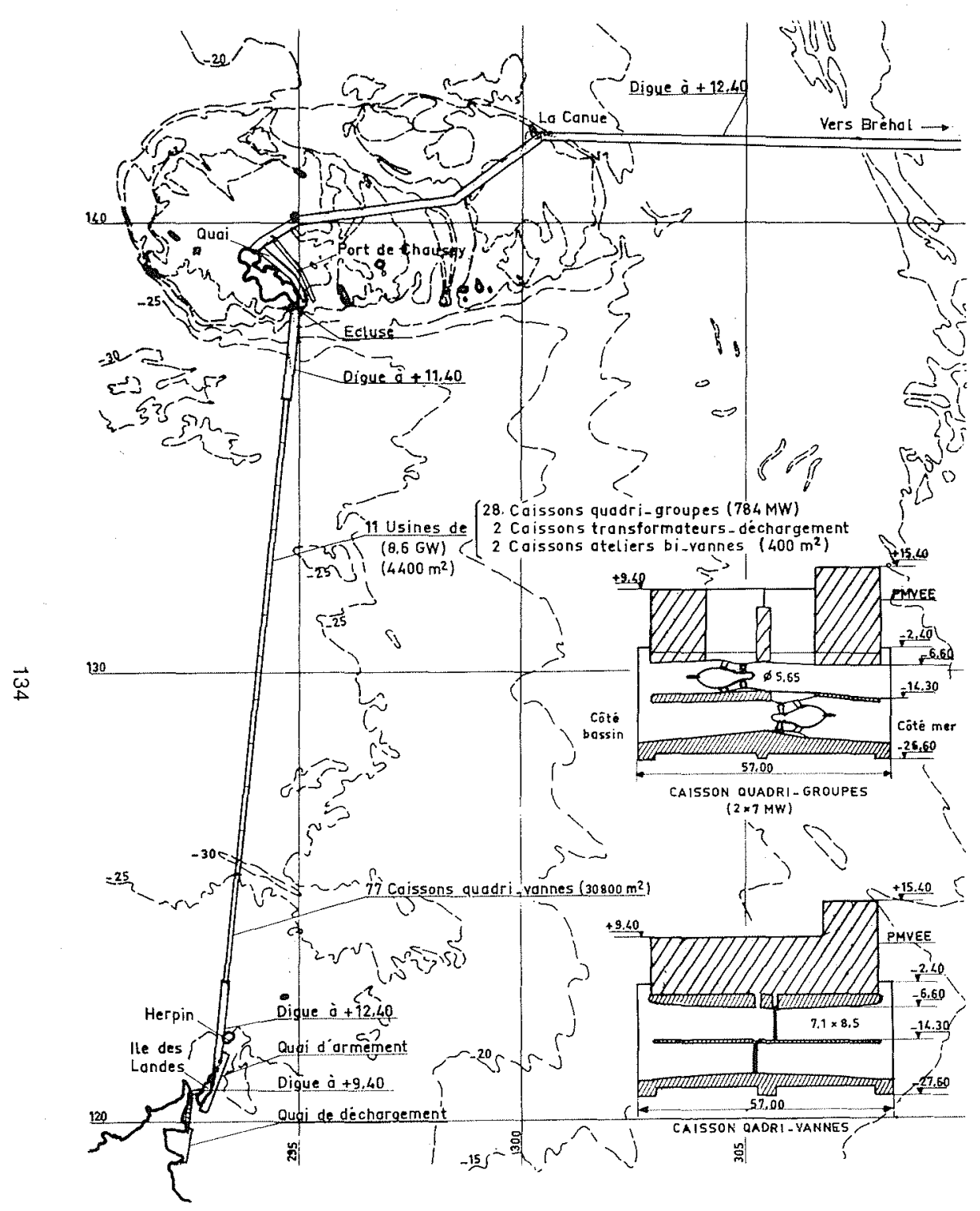

Cotes N.G.F. $(+6,60$ C.M.)
Echelle : 1175000

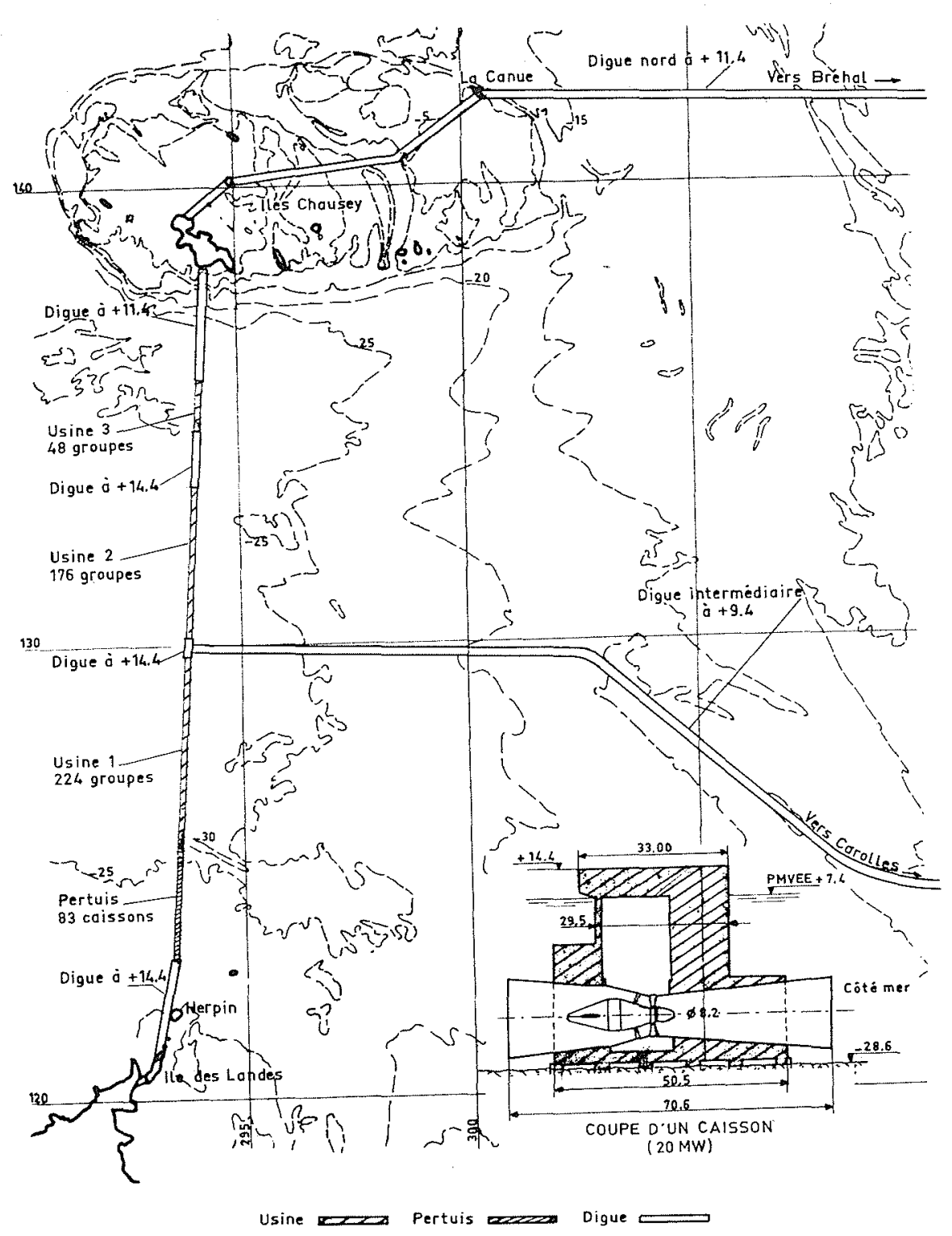

Cotes N.G.F. $(\omega 6,60$ C.M. $)$ 
$8,2 \mathrm{~m}$ ) et en tenant compte des résultats des premières campagnes de mesures géologiques, de courant et de houle. De plus fut examinée l'idée d'une digue intermédiaire permettant la mise en service anticipée de la moitié de l'usine. En fait ce projet pèche par la méconnaissance de la cote du bon rocher.

Le projet comporte 224 caissons bi-groupes (448 groupes, 8,96 GW) de 62000 tonnes (un caisson monogroupe, pas assez large vis-à-vis de ses autres dimensións, aurait une stabilité précaire). Leur implantation résulte d'un compromis entre la nécessité d'un fonçage des caissons à - $30 \mathrm{~m} \mathrm{NGF} \mathrm{du} \mathrm{fait} \mathrm{de} \mathrm{l'augmentation}$ de la taille des groupes $(-21 \mathrm{~m}$ pour les groupes de $10 \mathrm{MW}$ ) et la position des fonds rocheux supposée entre -27 et $-30 \mathrm{~m}$ (le rocher est en fait entre -30 et - $40 \mathrm{~m}$ ). De ce fait, il est proposé de construire trois usines (fig. 36) dont les radiers des caissons seraient calés à des cotes variant de -27 à $-31 \mathrm{~m}$, séparées par des digues en enrochements arasées à $+14,4 \mathrm{~m}$ dans les zones d'érosion importantes (fond à $-35 \mathrm{~m}$ ). Les 83 caissons-pertuis $\left(30000 \mathrm{~m}^{2}\right)$ sont disposés comme dans le projet E.D.F. 1955/56; ils sont foncés à des cotes variant de -24 à $-27 \mathrm{~m}$.

Compte tenu des problèmes posés par l'implantation de caissons-usines dans des zones où le rocher est à une cote très basse, une variante à 264 caissons $(528$ groupes, $10,56 \mathrm{MW}$ ) fut proposée en reportant l'usine plus au sud, en disposant de 16 caissons à la cote $-27 \mathrm{~m}$ et de 4 caissons à $-33 \mathrm{~m}$.

Le calendrier des travaux tient compte des résultats des études sur modèle réduit hydraulique, à savoir qu'il est préférable de commencer l'usine par la pointe du Grouin, et que l'exécution simultanée de la digue Nord est possible.

Cet avant-projet sommaire ne comprend pas d'estimation de délai ou de prix. Son intérêt essentiel est de mettre en relief les problèmes posés par les cotes du rocher et la nécessité d'enfoncer les groupes de $20 \mathrm{MW}$.

\subsection{Projet d'octobre 1957 [213e]}

L'usine est réalisée en échouant à la file les caissons bigroupes de 40000 tonnes (tirant d'eau $21,57 \mathrm{~m}$, $50,6 \mathrm{~m}$ de long et $47,5 \mathrm{~m}$ de large à la fondation). Les caissons sont prolongés par des trompettes métalliques de $10 \mathrm{~m}$ de long; ils sont plus petits que ceux prévus en février 1957 (base de $74,6 \mathrm{~m} \times 37,1 \mathrm{~m}$ ). Les caissonsusines ont une très faible largeur $(29,5 \mathrm{~m})$ au-dessus du niveau de basse-mer; ceci assure la stabilité de l'usine en dépit du faible cube de béton $\left(735 \mathrm{~m}^{3}\right.$ par mètre d'usine).

\subsubsection{Le PROJET G.T.M. 1957 (12 GW)}

Dans le projet E.D.F. 1955/56, le prix du génie civil de l'usine-caisson est approximativement le double de celui qu'il aurait été si l'usine était construite à sec comme celle de la Rance, il s'imposait donc d'examiner la réalisation par batardeaux. Ce procédé, de plus, facilite la mise en service progressive des groupes et l'exécution des bouchures, lesquelles seront aussi difficiles que celles de la Rance, du fait du très petit nombre de groupes en service aux moments critiques dans le cas d'une mise en service progressive des groupes.
Le projet de la Société des Grands Travaux de Marseille (G.T.M.) est une concrétisation des idées de L. Vantroys [214]. Il concerne uniquement l'édification des batardeaux nécessaires pour construire les $14 \mathrm{~km}$ d'usine. Pour atteindre la cadence de bétonnage de l'usine de $140000 \mathrm{~m}^{3}$ par mois de façon à l'achever en quinze ans, il faut disposer en permanence de $4 \mathrm{~km}$ d'enceinte batardée; le chantier serait alors constitué de 4 enceintes de $1 \mathrm{~km}$ de long sur $300 \mathrm{~m}$ de large, les batardeaux d'une enceinte, où le bétonnage est terminé, étant replacés immédiatement autour d'une nouvelle enceinte (fig. 37). Les batardeaux longitudinaux sont constitués par des caissons rectangulaires de $65.3 \mathrm{~m} \times 20 \mathrm{~m} \times 25 \mathrm{~m}$, posés sur des banquettes en tout venant de carrière arasées à la cote $-17,6 \mathrm{~m}$, protégées par des enrochements contre la houle côté mer. Certains caissons comportent des portes mobiles utiles pour les opérations de coupure; les batardeaux transversaux sont en gabions de palplanches pouvant s'enlever à sec lors de la mise en communication de deux enceintes contiguës.

Le programme de travaux est le suivant (figures 38 et 39):

- 6.5 ans : fermeture du bassin Sud et mise en service de $1 \mathrm{GW}$;

- 8,9 et 11 ans: mise en service de 3, 4 et $7 \mathrm{GW}$;

- 12 ans : fermeture du bassin Sud et mise en service de $8 \mathrm{GW}$.

- 15 ans : mise en service de $12 \mathrm{GW}$.

En milliards de francs 1957 , le devis estimatif du génie civil est :

— installations provisoires ............ 2,9

— épuisements ................... 32,5

— batardeaux longitudinaux . . ......... 136,8

- batardeaux transversaux .............. 21,6

soit $4,3.10^{9} \mathrm{~F} 1974$

193,8

Notons que L. Vantroys, en 1955, avait estimé ce travail à 200 milliards de francs [210] et le total de l'usine à 1000 milliards de francs $\left(25.10^{9} \mathrm{~F} 1974\right)$.

L'intérêt de ce projet c'est la disponibilité de $1 \mathrm{GW}$ au bout de 6,5 ans; ensuite 1 à $2 \mathrm{GW}$ supplémentaires seraient mis en service annuellement.

\subsubsection{L'AVANT.PROJET R.E.H. N 8 DE 1958 (12 GW)}

La campagne géologique a montré (fig. 31) que, sur la ligne pointe du Grouin-île de Chausey, sur la moitié de la longueur, les fonds rocheux sont à $-35 \mathrm{~m}$; en revanche, ils se situent autour de $-25 \mathrm{~m}$ sur la plus grande partie de la ligne pointe du Grouin-Les Huguenans, nouveau tracé proposé [215].

Le projet comporte (fig. 40) 600 groupes de $20 \mathrm{MW}$ et $32000 \mathrm{~m}^{2}$ de pertuis de vannage. Les ouvrages, construits à sec entre les batardeaux GTM, se répartissent en :

- 7 usines comprenant chacune 80 groupes et 6 pertuis de $12 \times 10 \mathrm{~m}$ au col du Venturi;

- 1/2 usine à 40 groupes et 4 pertuis;

- 2 ensembles de 50 pertuis de vannage de même dimension. 

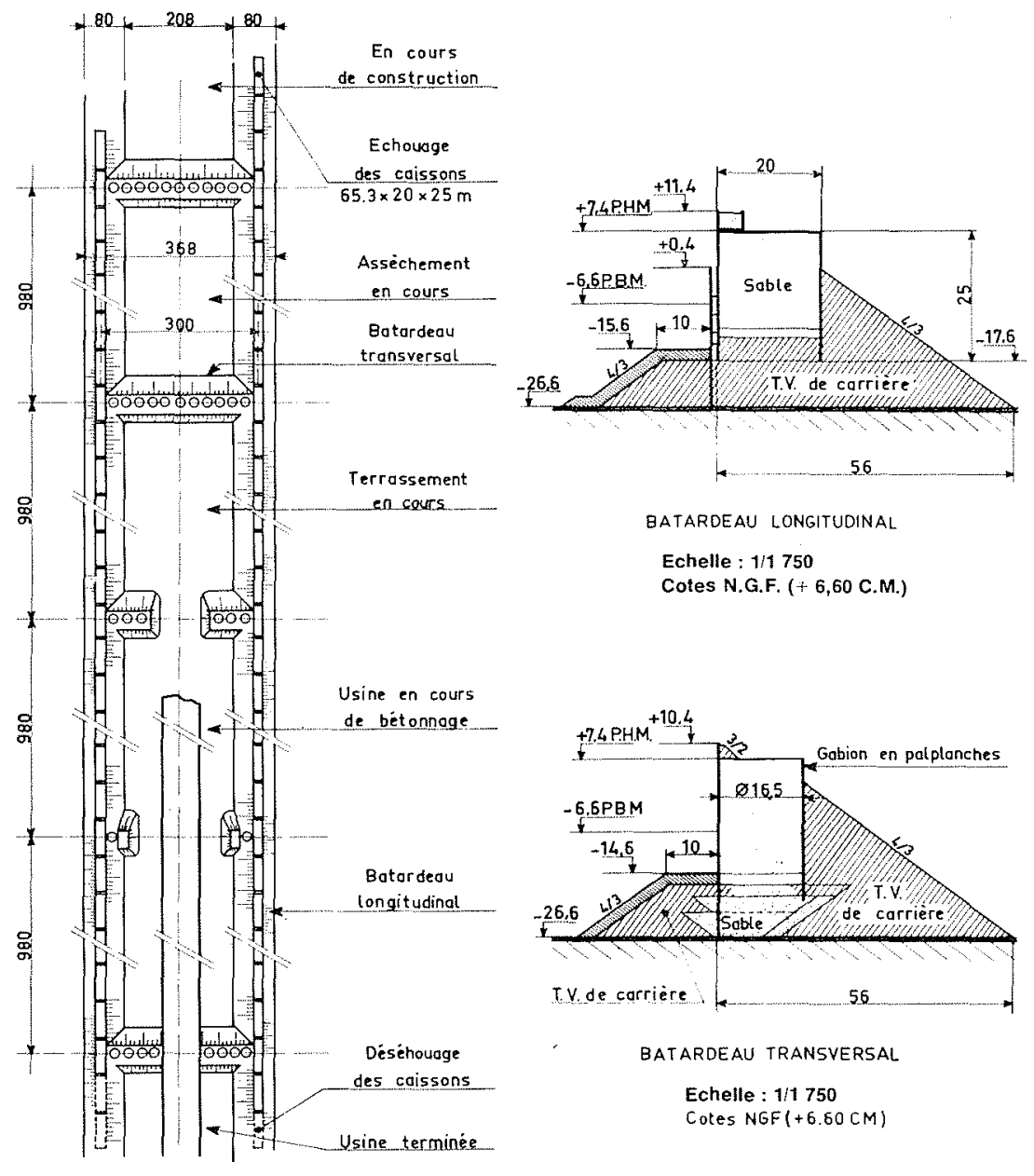

Echelle : 1/17 500

37/ Principe d'exécution des batardeaux du projet G.T.M. 1957

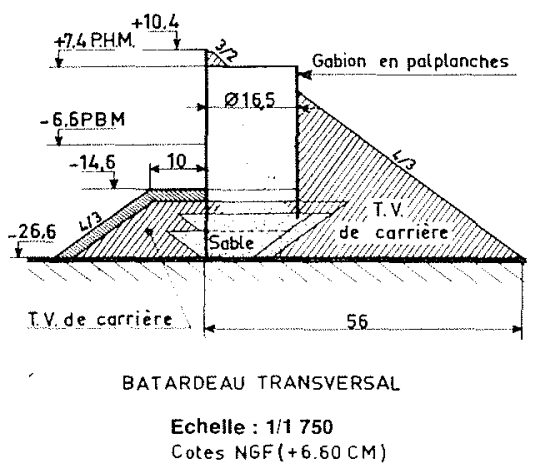

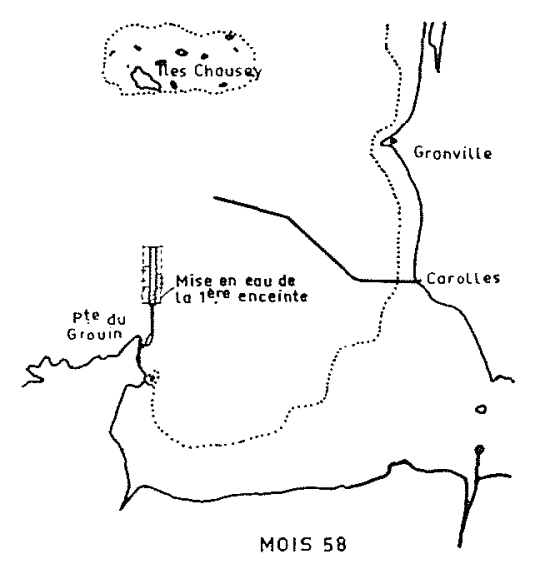

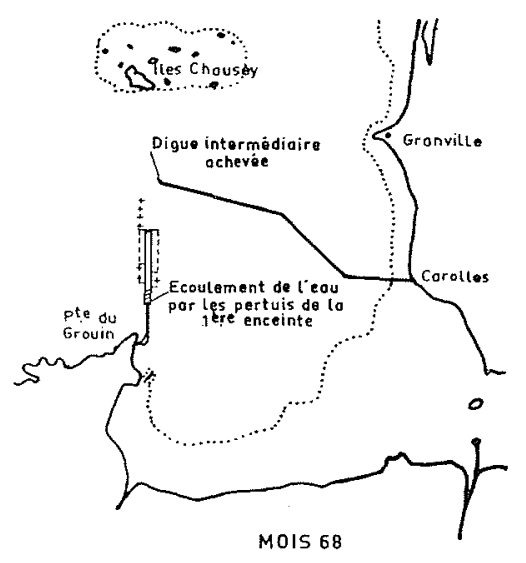

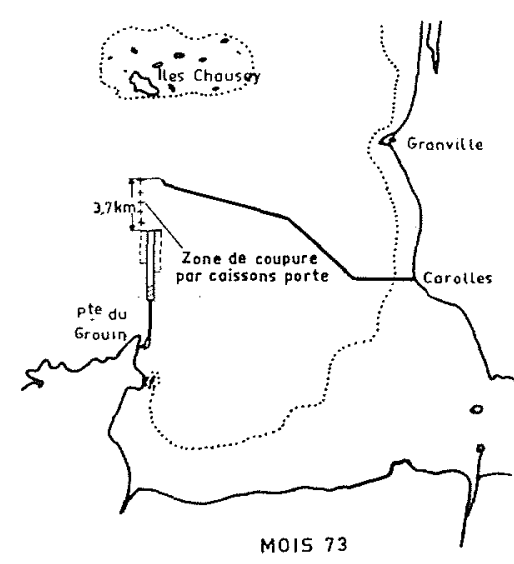

COUPURE DU BASSIN SUD

$4++++$ Coissons ò portes Pertuls

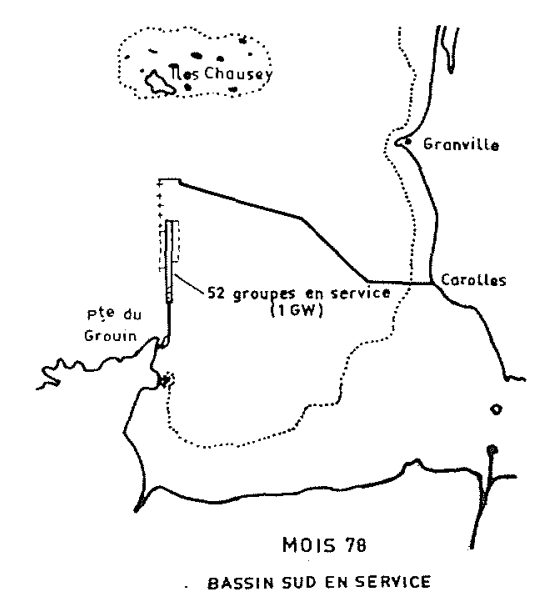

Echelle : 1700000
38/ Phases de travaux du projet G.T.M. 1957 Années 1 à 6,5 

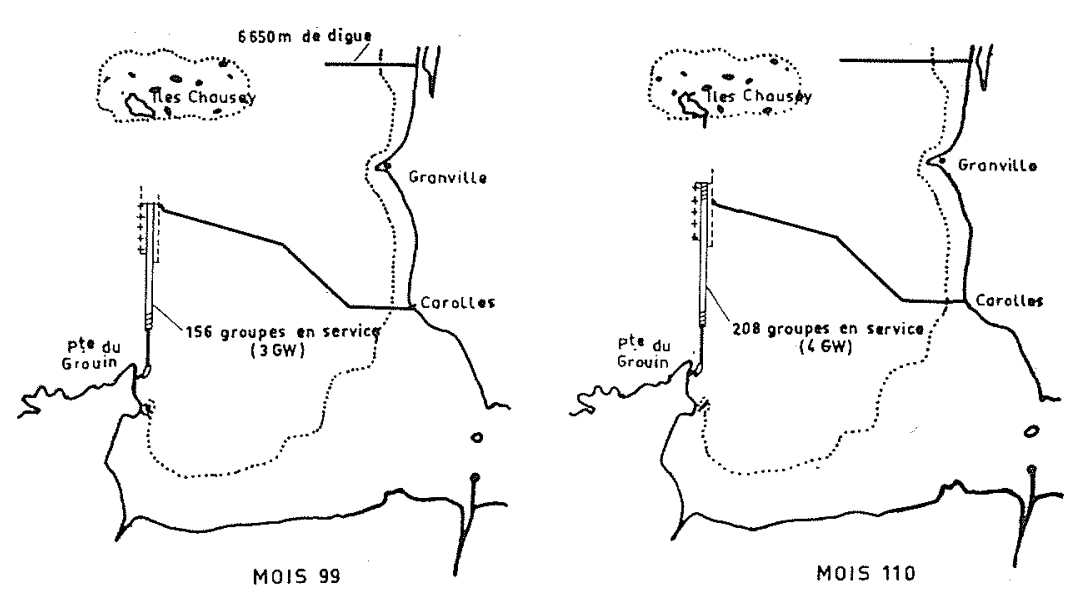

$\vec{\omega}$

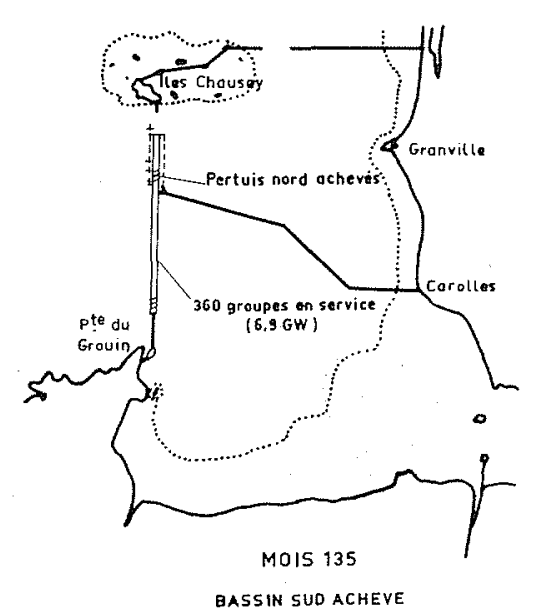

Echelle : $1 / 700000$

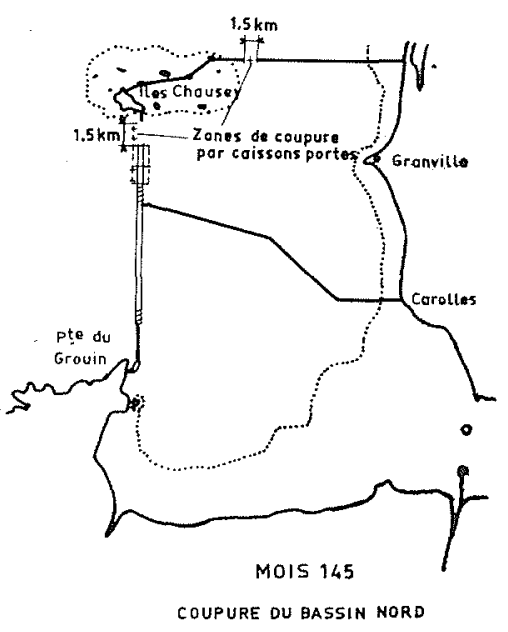

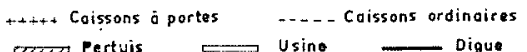

39/ Phases de travaux du projet G.T.M. 1957 Années 6,5 à 12

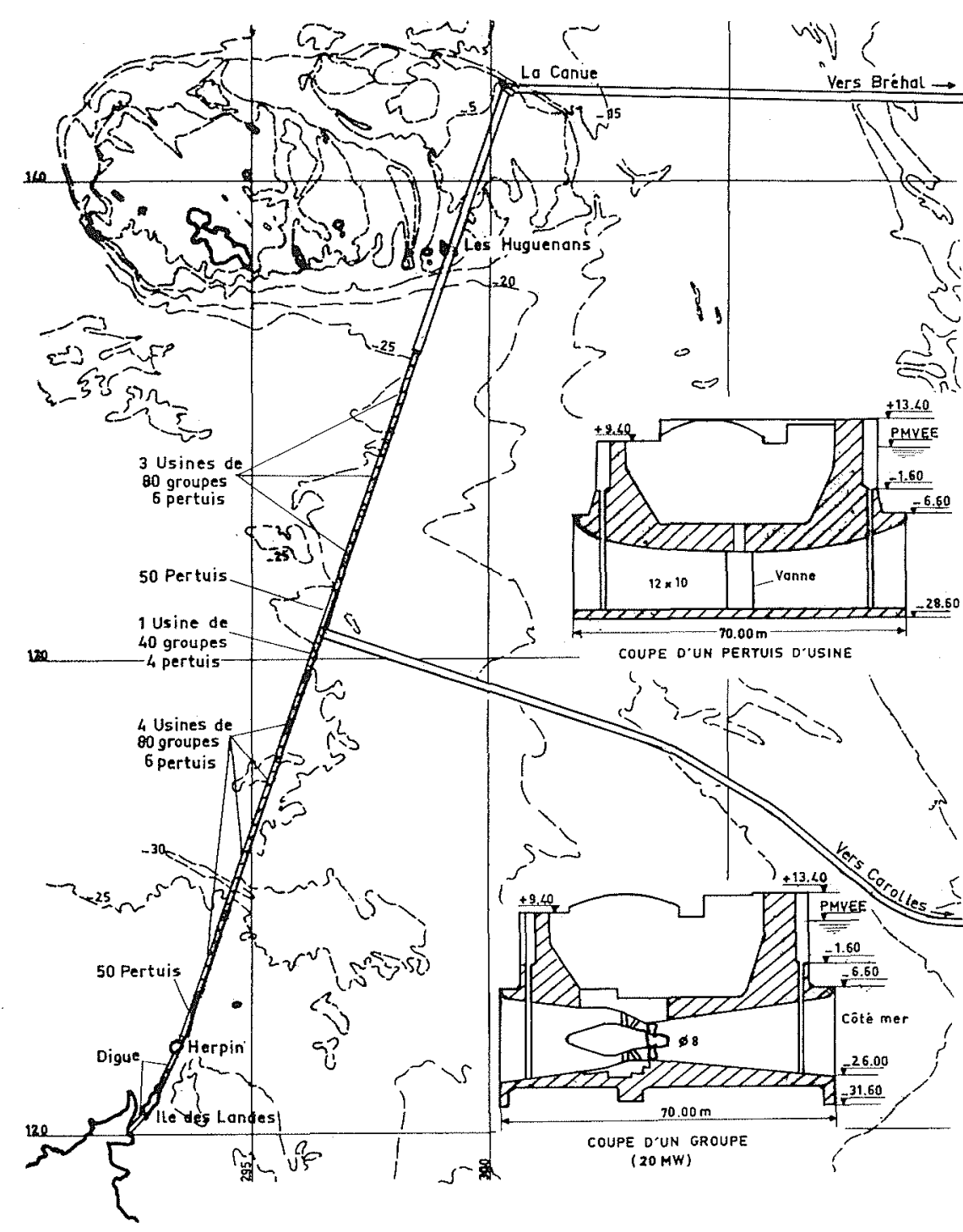

Usine à 80 groupes Usine à 40 groupes
Echelle : $1 / 175000$ Cotes N.G.F. $(+6,60$ C.M. $)$ 
Chaque usine est divisée en 2 demi-usines symétriques, comportant chacune 2 blocs identiques de 20 groupes constitués de 2 tranches de 10 groupes (200 MW) reliées en couplage-bloc avec un transformateur de $400 \mathrm{MVA}-380 \mathrm{kV}$. Du point de vue électrique chaque usine peut être considérée comme un ensemble de 8 groupes de $200 \mathrm{MW}$ réunis à 4 transformateurs de $400 \mathrm{MW}$. La puissance pourrait être évacuée en réalisant une des trois solutions suivantes:

- totalité par la pointe du Grouin;

- bassin Sud par la pointe du Grouin et bassin Nord par Bréhal ;

- bassin Sud par la pointe du Grouin et la pointe de Carolles et bassin Nord par Bréhal.

Les quantités de matériaux à mettre en ceuvre seraient les suivants :

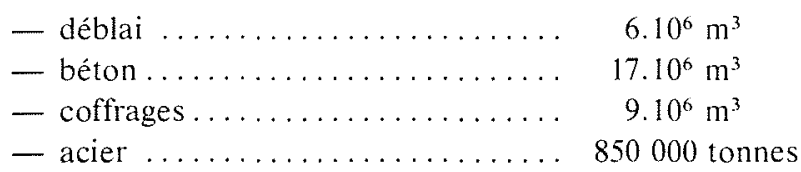

\subsubsection{Réflexions sur les projets postérieurs à 1955}

La période 1956-1958 est caractérisée par l'avènement des groupes bulbes, d'abord de $10 \mathrm{MW}$ type Rance, puis de $20 \mathrm{MW}$. Actuellement il ne serait pas interdit d'envisager des groupes de $50 \mathrm{MW}$ bien que ceux-ci posent des problèmes d'efforts sur les paliers.

La structure de l'usine s'organise autour des considérations suivantes:

- La hauteur de l'usine est fixée par la cote de fondation; il y a intérêt à utiliser au maximum la hauteur de l'usine, d'où l'idée des groupes superposés, dans la limite des effets de la houle; dans le cas d'une usine exécutée à sec, l'approfondissement du terrain est relativement peu onéreux; or on sait que le bon rocher est à une cote profonde, ce qui était une gêne devient un avantage avec des groupes de $40 \mathrm{MW}$, dont on peut estimer le diamètre à $12 \mathrm{~m}$ environ.

- La longueur de l'usine est inversement proportionnelle à la puissance linéaire, donc sensiblement inversement proportionnelle à la racine carrée de la puissance unitaire des groupes; il en est donc de même pour le prix des batardeaux provisoires.

- La largeur de l'usine est fonction de la longueur des galeries d'alimentation du groupe, sensiblement proportionnelle à la racine carrée de la puissance élémentaire; compte tenu des sous-pressions, pour une profondeur donnée, il existe une largeur optimale de l'usine assurant la stabilité avec le minimum de poids.

Tous les projets sont basés sur un délai d'exécution de quinze ans; une possibilité de mise en service des premiers groupes après six ans est apparue. La digue intermédiaire, à partir de Carolles, est économiquement intéressante, d'autant plus qu'elle accède directement aux carrières de Carolles.

La nature du sol impose des sujetions dont les projets ne tiennent pas souvent compte :
- Le tracé Ouest traverse une zone de transition géologique; au sud, le rocher est à $-26,-32 \mathrm{~m}$; l'épaisseur des sédiments est faible (2 à $3 \mathrm{~m}$ ); au nord, le rocher est profond $(-40 \mathrm{~m})$ et recouvert d'une épaisse couche de sable vaseux ( 8 à $9 \mathrm{~m}$ ).

- Sur le tracé Nord, le rocher est à $-24 \mathrm{~m}$, recouvert de $12 \mathrm{~m}$ de sable très coquillé.

La baie n'est pas très riche en matériaux de qualité (sable propre, galets,...). Il ne semble pas qu'un déplacement de quelques kilomètres des tracés soit intéressant, sauf à chercher les grands fonds rocheux plus au large dans le cas de groupes de grandes puissances.

La hauteur maximale de la cote de l'eau peut être fixée à $+7,4 \mathrm{~m} \mathrm{NGF}(+14 \mathrm{~m} \mathrm{CM})$; la houle peut atteindre une hauteur de $6 \mathrm{~m}$. Pour n'être absolument pas franchie, l'usine devrait culminer à +14,4 $\mathrm{m} \mathrm{NGF}$ $(+21 \mathrm{~m} \mathrm{CM})$, mais ceci sans profiter des ouvrages anti-franchissements mis au point depuis 1958. La houle reste cependant le paramètre sur lequel il y aurait encore a etudier, du triple point de vue : prévision, efforts sur l'usine, fluctuations de puissance.

Les études sur modèles réduits hydrauliques ont montré l'intérêt de construire la digue Nord avant l'achèvement de la digue Ouest, de façon à réduire les courants dans les brèches. De même, il y aurait intérêt à construire d'abord la digue intermédiaire.

En 1955, on assiste au triomphe des méthodes de construction par caissons; en 1958, cette méthode perd de son intérêt, car le fonçage du caisson devient très onéreux si la cote du rocher est inférieure à $-30 \mathrm{~m}$. Le procédé de construction de batardeaux, par caisson en béton armé échoués sur banquette, a alors des avantages certains; toutefois un seul avant-projet a été étudié ; il est possible qu'il pourrait être amélioré en utilisant, par exemple, des caissons métalliques.

Il reste cependant des questions sans réponses

- l'entraînement des sédiments fins dans les brèches;

- l'étanchéité des batardeaux dans les zones à forte épaisseur de sédiment.

\section{Les Minquiers}

Pour décrire les Minquiers, laissons parler A. de Chevremont [2] : "Quant aux plateaux rocheux, les uns, comme Chausey, sont devenus des archipels bas et pour ainsi dire émiettés; les autres, comme les Minquiers, plus avancés dans leur évolution vers l'abîme, ne laissent voir qu'à mer basse le plus grand nombre des sommets de leurs collines primitives; à mer haute ce sont plus guère que des récifs sur lesquels blanchit la lame".

Utiliser ce plateau comme appui des digues a tenté les ingénieurs; ils ont ébauché, en 1955, un projet de bassin de $600 \mathrm{~km}^{2}$ limité par $63 \mathrm{~km}$ de digues partant du cap Fréhel et de la pointe du Decollé vers les Minquiers; l'usine (12 GW) serait installée face à celle de Chausey. La digue morte, entre le cap Fréhel et les Minquiers, protégerait les deux usines contre la houle 


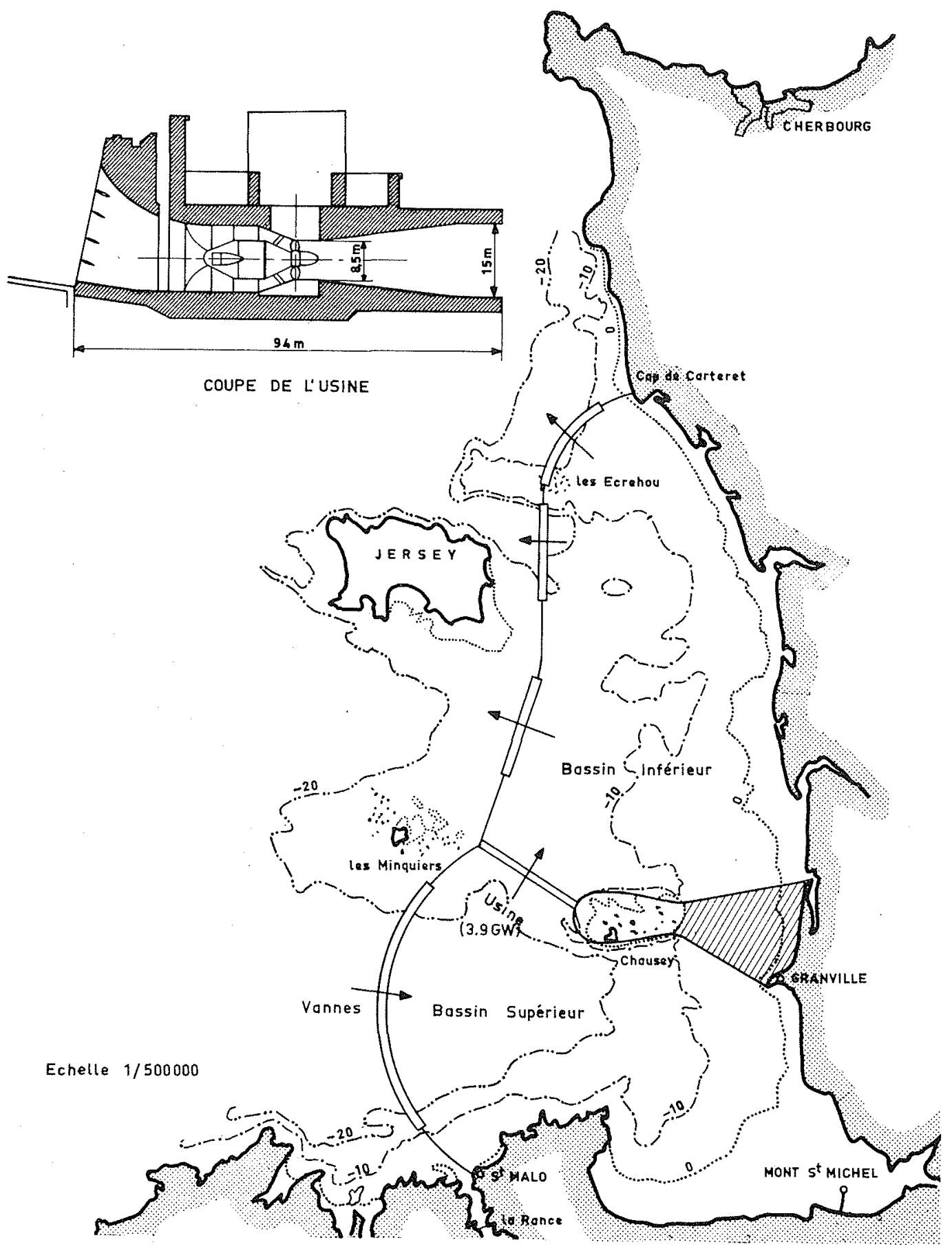

41/ Le projet d'usine de A. Caquot (1971)

du large; on retrouve ici la crainte des effets de la houle. Mais actuellement, compte tenu de la connaissance du processus d'alimentation hydraulique de l'usine, toutes réserves sont à faire sur l'idée de construire deux usines si proches; on sait combien un projet Chausey de $12 \mathrm{GW}$ commence à réduire l'amplitude locale de la marée; demander plus d'énergie du site est techniquement sans intérêt.

En 1971, Albert Caquot [107] propose de construire une très grande usine à deux bassins de $1100 \mathrm{~km}^{2}$, séparés par un ouvrage comportant une usine, entre les Minquiers et Chausey, équipée de 6 GW (200 groupes de $30 \mathrm{MW}$ ) fonctionnant toujours avec le même sens de l'écoulement (fig. 41). Les autres caractéristiques du projet sont :

- débit des vannes $500000 \mathrm{~m}^{3} / \mathrm{s}$

- production. $34 \mathrm{TWh} / \mathrm{an}$

$3,9 \mathrm{GW}$

- longueur des digues............

$35 \mathrm{~km}$

- longueur des vannes ..........

$50 \mathrm{~km}$

Les ouvrages extérieurs ont été dessinés en suivant une ligne d'égal marnage. L'idée de base du projet est d'essayer d'assurel une puissance garantie, d'où l'utili- 
sation du cycle de Belidor. Le projet est évalué à environ 25 milliards de francs 1974.

Le projet est conçu pour être à but multiple. Il est proposé d'édifier une plateforme de 12000 ha entre l'archipel de Chausey et Granville pour y installer un complexe industriel, un équipement nucléaire, un port en eau profonde.

Il est permis de s'interroger si, pour réaliser une usine marémotrice dont le but principal est de fournir de l'énergie non polluante, il faut sacrifier le site au profit d'un complexe industriel. De plus, est-il bien nécessaire de demander à cette usine une puissance garantie, au lieu de l'utiliser comme usine de pointe, surtout à une époque où l'équipement de base sera nucléaire. Il est aussi un argument écologique à opposer à l'usine : dans chacun des bassins, le niveau de l'eau ne variera que de $5 \mathrm{~m}$ environ, au lieu de $13 \mathrm{~m}$ actuellement; ce qui signifie basse-mer et pleine-mer permanentes dans les bassins inférieur et supérieur. En fait ce projet ne satisfait pas aux exigences de 1974. Nous croyons savoir qu'un projet fournissant de l'énergie de pointe, fonctionnant couplé avec des usines nucléaires, est en cours d'élaboration.

Enfin rappelons que le projet des Minquiers poserait un problème juridique depuis la décision de la Cour Internationale de La Haye attribuant à Jersey la souveraineté sur l'archipel des Minquiers. Mais il ne semble pas que ce problème juridique soit insoluble, puisque le représentant britannique a parlé en ces termes dans le plaidoyer devant la Cour Internationale: «Nous avons consulté nos experts sur ces projets, et leur réponse est qu'il est parfaitement exact qu'il s'agit là non de rêves d'ingénieur mais de projets réalisables. Même le plus ambitieux de ces trois projets, semble-t-il, est techniquement réalisable, bien qu'un grand nombre de difficultés pratiques doivent être résolues. Sous réserve des garanties nécessaires, jamais nous ne songerions à exciper de notre souveraineté sur les Minquiers pour empêcher la réalisation de travaux hydro-électriques qui pourraient être au bénéfice de la France. »

\section{Bibliographie}

\section{Documents publiés}

[1] BELLIDOR. - "Architecture hydraulique ou l'art de conduire, d'élever et d'aménager les eaux pour les différents besoins de la vie ». Tome I, livre II, ch. I, p. 304-309, 1737

[2] Chevrevont (A. de). - "Les mouvements du sol sur les côtes uccidentales de France et particulièrement dans le golfe Normand-Breton ». Paris, 1822

[3] MAYNARD (E.). - Etude sur l'utilisation des marées pour la production de la force motrice. Revue Générale de l'Electricité : $(2,9,16,23$ et 30 novembre, $7,14,21$ et 28 décembre 1918), p. 653-658, 697-715, 749-762, 793$802,823-843,865-877,903-914,947-959,977-1007$.

[4] GibRat (R.). - Notes sur la production d'électricité au moyen des marées. Revue de l'Industrie Minérale, $n^{\circ} 489$ (août 1944), p. 309-352.

[5] Allard (P.). - Forme et énergie de l'onde-marée de vive-eau entre les Heaux de Bréhat et le cap de la Hague. Annales de Géophysique, tome VII, no 1 (janvier-mars 1951), p. 9-44.
[6] Allakd (P.). - Bilan énergétique de la marée de viveeau pour la Manche entière. Bulletin du C.O.E.C., $\mathrm{n}^{\circ} 8$ (septembre-octobre 1952), p. 307

[7] Govgenheim (A.). - Sur la fréquence des coefficients de marée. Bulletin du C.O.E.C. (mars 1953), p. 119-133

[8] Gibrat (R.). - L'énergie des marées. Bulletin de la Société Française des Electriciens, $7^{\mathrm{e}}$ série, tome III, $\mathrm{n}^{\prime \prime}$ 29 (mai 1953), p. 283-332.

[9] Philipponneau (M.). - La Baie du Mont-Saint-Michel. Mémoires de la Société Géologique et Minéralogique de Bretagne, tome XI (1955).

[10] GRaINdor (M. J.). - Méthode de prospection géologique sous-marine mise au point dans la baie du MontSaint-Michel. Bulletin de la Société Linnéenne de Normandie, $9^{e}$ série, 9 vol. (1956/58), p. 30

[11] Communication aux $4^{\text {e }}$ journées de l'Hydraulique "Les Énergies de la mer». Société Hydrotechnique de France, Paris (juin 1956) :

[11a] Dieulot (G.). - Les enregistrements de houle à l'aide d'un sondeur inversé aux îles Chausey, p. 85-94.

[1 $1 b]$ Bonvefille (R.). - Etude de la marée en Manche, p. 166-175.

[11c] VantroYs (L.). - Le régime des marées dans la Manche. Influence de la rotation de la Terre. Rapport sur les travaux de la Commission d'Etudes, p. 176-181.

[11d] BIESEL (F.). - Quelques remarques à propos de l'influence de la force de Coriolis sur les marées de la Manche, p. 182-187.

[11e] Dieulot (G.). - Mesures de courants en baie du Mont-Saint-Michel, p. 209-217.

[11f] Kammerlocher (L) et Duclos (J.). - Evolution constructive des groupes de l'usine de la Rance, p. 511-521.

[12] Graindor (M. J.) et Roblot (M. M.). - Observations sur les îlots rocheux situés au nord de Granville. Bulletin de la Société Géologique de France, 6e série, tome 6 (1956), p. 115-126.

[13] Debrser (J.) et Fondeux (C.). - Application des méthodes granulométriques à l'interprétation d'une série détritique actuelle, étude de la sédimentation entre Granville et Chausey. Revue de l'Institut Français du Pétrole, vol. XI, no 5 (mai 1956), p. 551-572.

[14] Graindor (M. J.). - Observations sur les gneiss des Ecrehous. C.R. sommaire des Séances de la Société Géologique de France, $\mathrm{n}^{\circ} 5$ (11 mars 1957), p. 89.

[15] Graindor (M. J.) et Roblot (M. M.). - Géologie des Minquiers. Premières observations. Bulletin de la Société Géologique de France, $6^{*}$ série, tome 7 (1957), p. 118-221.

[16] Mémoires et Travaux de la Société Hydrotechnique de France, vol. II (1957)

[16a] Rath (R.) et Surel (G.). - La corrosion par la mer du matériel des usines marémotrices, $p$. 139-153.

[16b] BonNefille (R.). - Etude expérimentale de l'influence de la force de Coriolis sur la propagation de la marée dans la Manche, $p$ 154-161.

[17] Graindor (M. J.). - Sur la présence de gros blocs encastrés dans les sables du Golfe normanno-breton. C.R.A.S. (21 décembre 1958).

[18] VANTROYS (L.) - - "Le remous d'un ouvrage dans une mer à marée ". Thèse soutenue à Paris le 23 février 1957. Imprimerie Nationale (1958).

[19] Vaytroys (L.), Magnien (M.) et Barret (P.). - Influence de la houle sur le comportement des groupes marémoteurs. S.H.F. 5 * Journées de l'Hydraulique, p. 195-208, Aix-en-Provence (26-27 juin 1958)

[20] VANTroYS (L.). - Les divers aspects mathématiques dynamiques ou cinématiques du problème des marées. Bulletin du C.O.F.C., no 8 (sept.-oct. 1958), p. 469-483; no 9 (nov, 1958), p. 541-558; no 10 (déc. 1958), p. 657 690. 
[21] Valembois (J.) et Bonnefille (R.). - Etude de l'action de la force de Coriolis sur la propagation des marées en fonction de l'étendue de la zone marine considérée. La Houille Blanche, $\mathrm{n}^{\circ} 5$ (août 1959), p. 568-585.

[22] Graindor (M. J.). - Une méthode de géologie sousmarine. Revue de Géographie Physique et de Géologie Dynamique, vol. II, fasc. 1 (1959), p. 29-34.

[23] Graindor (M. J.) et Marie (P.). - Le Sénonien inférieur au S.-S.-W. des îles Chausey. C.R. sommaire des Séances de la Société Géologique de France, $\mathrm{n}^{0} 3$ (2 mars 1959), p. 56

[24] Munk (W. H.) et MAC Donald (G. F. J.). — « The rotation of the earth, a geophysical discussion ». Cambridge University Press (1960), p. 198-230.

[25] Gohiv (F.). - Détermination des dénivellations et des courants de marée. VII ${ }^{\mathrm{e}}$ Congrès du Coastal Engineering (1960), p. 485-509.

[26] Bourcart (J.) et Bollot (G.). - La répartition des sédiments dans la baie du Mont-Saint-Michel. Revue de Géographie Physique et de Géologie Dynamique (2) vol. III, fasc. 4 (1960), p. 189-199.

[27] La Rance. La Houille Blanche, $\mathrm{n}^{\circ} 2$, mars-avril 1962 :

[27a] Dubors (R.). - Les essais du groupe marémoteur expérimental de Saint-Malo, p. 131-140.

[27b] Chevalier (J.). - Comparaison entre les rendements industriels et modèle pour le fonctionnement en turbinage inversé des groupes bulbes de Cambeyrac et Saint-Malo, p. 141 152.

[27c] Licheron (S.). - La lutte contre la corrosion du matériel des usines marémotrices, p. 166 178.

[27d] Legrand (R.) et Lambert (M.). - Mesures électrochimiques appliquées à l'étude de la protection cathodique des ouvrages de la Rance, p. 179-195.

[27e] Sanhes (J.). - Protection contre la corrosion marine de la station marémotrice expérimentale de Saint-Malo, p. 196-206.

[27f] Bonnefille (R.) et Voyer (F.). - Calcul de la propagation de la marée dans une zone côtière, p. $225-254$.

[27g] Chabert diHiéres (G.). — Réglages et exploitation de la plaque tournante de Grenoble, p. 244-254.

[27h] Gauthier (M.). - Eléments nouveaux dans le calcul des cycles des usines marémotrices, $p$. 269-275

[27i] Caseau (M.). - Classification des cycles d'une usine marémotrice, p. 276-283.

[28] BonNefille (R.). - Etude énergétique de la marée dans le golfe de Saint-Malo à partir des observations en nature. Bulletin du C.R.E.C., $\mathrm{n}^{\circ} 5$ (1963), p. 53-65.

[29] Mémoires et Travaux de la Société Hydrotechnique de France, vol. 1, 1964 :

[29a] Bonnefille (R.) et Jeannel (M.). - Etude sur modèle réduit de la coupure de la Rance, $p$ $42-50$.

[29b] Duhoux (L.). - Fermeture de la Rance. Déroulement des travaux et analyse des observations, p. 51-68

[30] Bernstein (L. B.). - Prilivnye elektrostantsii v sovremennoi energetike, Moscou (1962). Traduit en anglais sous le titre "Tidal Energy for Electric Power Plant ", par Israel Program for Scientic Translation, Jerusalem (1965).

[31] Bonnefille (R.). - Les observations de marée effectuées par Electricité de France dans le golfe de Saint-Malo. Proceedings of the Symposium on Mathematical Hydrodynamical Methods of Physical Oceanography, Institut für Meereskunde der Universität, Hamburg (septembre 1965).

[32] Gibrat (R.). - «L'Energie des Marées». Collection la Science Vivante. Presses Universitaires de France, Paris (1966).
[33] La Rance, une grande réalisation inédite. Revue Française de l'Energie, 183 (sept.-oct. 1966).

[34] HyaCinTHE (J. L.). - Sur le modèle mathématique des mers littorales, application à la Manche. C.R.A.S., t. 263 (25-7-1966), série A, p. 181-184.

- Courbes relatives aux dénivellations de marée dans la Manche obtenues à l'aide d'un modèle mathématique. C.R.A.S., t. 263 (22-8-1966), série A, p. 271 274

- Courbes relatives aux courants de marée dans la Manche obtenues à l'aide d'un modèle mathématique. C.R.A.S., t. 263 (22-8-1966), série A, p. 292-295.

[35] Blanc-Ferraud (P.) et Huard (P.). - Gestion optimale de l'usine de la Rance par une méthode de programmation dynamique. E.D.F. Bulletin de la Direction des Etudes et Recherches, série B, no 3 (1967), p. 5-18.

[36] Bonnefille (R.) et Chabert D Hières (G.). - Etude d'un modèle tournant de mer littorale, application à l'usine marémotrice des îles Chausey. La Houille Blanche, $\mathrm{n}^{\circ} 6$ (1967), p. 651-658.

[37] Bonnefille (R.), Jeannel (M.) et Salomone (A.). Etude sur modèle réduit de l'usine marémotrice de la Rance. Bulletin de la Direction des Etudes et Recherches, série $\mathrm{A}, \mathrm{n}^{\circ} 2$ (1967), p. 5-106.

[38] Bonvefille (R.). - «Contribution théorique et expérimentale à l'étude du régime des marées". Thèse. Université de Grenoble (16 octobre 1968).

[39] Fiability of tidal power development in the Bay of Fundy. Board Report and Committee Report. Atlantic Tidal Programming Board (octobre 1969).

[40] Gibrat (R.). - Les usines marémotrices et le Canada Revue Francaise de l'Energie, $\mathrm{n}^{0} 233$ (juillet-août 1971), p 453-469.

[41] Tidal Power, édité par Gray et Gashin, Prenum Press (1972) :

[41a] Lawton (F. L.). - Tidal Power in the Bay of Fundy, p. 1-104.

[4lb] LaWTON (F. L.). - Economics of tidal power, p. $105-130$.

[4lc] Mauboussin (C.). - L'usine marémotrice de la Rance, p. 189-214.

[4ld] Bernstern (L.). - Kislaya Guba experimental tidal power plant and problem of the use of tidal energy, p. 215-238.

[4le] WILSON (W.). - Tidal power from Cook Inlet, Alaska, p. 239-256.

[4lf] Fentzloff (H.). - The tidal power plant « San José », p. 257-265.

[42] Fortin (J.). - " Les sédiments et la dynamique sédimentaire dans l'archipel des îles Chausey ». Thèse de $3^{\text {e }}$ cycle, Université de Caen, 17 juillet 1972.

[43] KaUfMANN (A.). - " Méthodes et modèles de la recherche opérationnelle", tome 2. Dunod, Paris (1972).

[44] Six ans d'exploitation de l'usine marémotrice de la Rance. La Houille Blanche, numéro spécial (2-3/1973):

[44a] Gandon (M.), Guillaumain (M.) et DE LaRQUIER (M.). - Exploitation de l'usine de la Rance. Méthode et résultats, p. 131"144.

[44b] GibRat (R.). - L'énergie marémotrice dans le monde; l'usine marémotrice de la Rance et l'environnement, p. 145-150.

[44c] Duhoux (L.). - Comportement en service des principaux ouvrages de génie civil de l'usine de la Rance, p. 153-161.

[44ll] Lefrançois (J.) et Marolleau (Y.), - Fonctionnement de l'usine de la Rance. Comportement du matériel électromécanique, p. 163 170

[44e] Bonnefille (R.) et SAlomone (A.). - Comparaison de quelques mesures en nature et sur modèles réduits hydrauliques, p. 171-177.

[44f] Cotillon (J.). - Les groupes bulbes : de Röstin en Avignon: l'essor d'une technique, $\mathrm{p}$. $179-200$ 
[44g] BEsLiN (M.) et al. - Idées actuelles sur la conception des groupes bulbes. Exemple de Caderousse, p. 201-215.

[44h] ANDRE (H.) et al. - De Cambeyrac à Strasbourg : quinze ans d'exploitation des groupes bulbes, p. 217-227.

[44i] CASACCi (S.) et al. - Les groupes bulbes; projets et perspectives, p. 229-246.

[44j] FARAL (M.). - Les différents types de protection contre la corrosion mis en auvre à l'usine marémotrice de la Rance, p. 247-250.

[44k] Leborgne (M.). - Comportement des métaux à l'usine marémotrice de la Rance, p. 251256

[44l] Legrand (R.) et Lambert (M.). - Bilan de protection cathodique à l'usine marémotrice de la Rance, p. 257-262.

[44m] Legrand (J.). - Etudes de matériaux susceptibles de résister à la corrosion par l'eau de mer, p. 263-269.

[45] Bernstern (L.). - Energy of the Northern seas. Marine Technology, Society Journal, 1973, n'2, p. 24.

[46] WILSON (E. M.). - Energy from the sea tidal power. Underwater Journal (août 1973), p. 175-187.

[47] Advenier (G.). - Aspect juridique de la réalisation de l'usine marémotrice de la Rance. Chronique. Cahiers Juridiques de l'Electricité et du Gaz, p. $489-506$ et 541-570 (octobre 1973).

[48] Cotillon (J.). - La Rance: Six years of operating a tidal power plant in France. Water Power (octobre 1974), p. 314-322.

\section{Documents non publiés}

\section{La Rance et divers}

[101] Baies de l'Arguenon et Lancieux. Etude théorique des cycles maximum d'énergie le jour. Neyrpic R 3130 (mars 1952).

[102] Note sur l'influence de la houle sur l'exploitation de l'usine marémotrice de la Rance. Direction des Etudes et Recherches, S.E.R., HR/2937 (19 avril 1956).

[103] Aperçu sur le projet d'usine marémotrice américanocanadien de Passamaquody. Direction de l'Equipement, S.E.P.H. (mars 1960).

[104] Sables de mer de la région de Saint-Malo. R.E.H. n" 8 , note 31 (29 mars 1960).

[105] Modèle mathématique de la Rance. Direction des Etudes et Recherches, C.R.E.C., HF/0289 (juillet 1965).

[106] Simulation de la marche de l'usine de la Rance. Direction des Etudes et Recherches, C.R.E.C., HF/0351 (mars 1966).

[107] Caquot (A.). - « La baie du Mont-Saint-Michel, grande source d'énergie» (février 1971)

[108] L'usine marémotrice de la Rance. Direction de l'Equipement, D.E.P.H. (juillet 1974).

[109] Rapports SOGREAH :

[109a] Cycles Rance, rapport $\mathrm{n}^{\prime 1}$, Documents de base. R 8742 (juin 1964).

[109b] Cycles Rance, rapport n" 2, Programme d'optimisation. R 9352 (octobre 1966).

[109c] Etude de la restructuration du programme Rance. R 9475 (février 1967).

\section{Chausey}

[201] Usine marémotrice de la Baie du Mont-Saint-Michel. Société l'Energie des Marées, Paris (septembre 1942).

[202] Essais de détermination par sismique réfraction de la nature du substratum autour de la baie du Mont-SaintMichel. Compagnie Générale de Géophysique, (5-7 mai 1952).
[203] Etude par prospection électrique de l'épaisseur des sables de grève aux îles Chausey. Compagnie Générale de Géophysique (17 juin-13 août 1952)

[204] Compte rendu de la mission de reconnaissance effectuée du 24 au 31 mars 1953.

[205] Détermination par sismique réfraction en mer de la nature du substratum au sud-est des îles Chausey. Compagnie Générale de Géophysique (26-31 mars et 14-20 avril 1953)

[206] Dievlor (G. W.). - Mesures de courants en baie du Mont-Saint-Michel, campagne 1953 (novembre 1953, novembre 1954).

[207] Mesures de courants, campagnes 1953-54. Chausey, tome I, notes explicatives et cartes.

[208] Rapports Neyrpic-SOGREAH :

[208a] Note sur les premiers essais d'amortissements des variations de pression dues aux clapotis à l'aval de l'usine, R 3610 (novembre 1953).

[208b] Etude de l'influence sur l'agitation d'un ouvrage immergé situé à plusieurs centaines de mètres de l'usine. R 3777 (juin 1954).

[208c] Variations de pression dues au clapotis à l'aval de l'usine. R 3975 (février 1955).

[208d] Etude de la répartition des pressions dues à la gifle le long d'une paroi verticale. (Notes de février, mars, avril et mai-juin 1956.)

[208e] Essais de prévision des caractéristiques des vagues dans la région des îles Chausey. R 4310 (mai 1956).

[208f] Analyse harmonique de la houle en mer et de la houle modèle. R 4345 (août 1956).

[208g] Etude de la réflexion de la houle sur un parement usine comportant une brisure de pente. R 4478 (mars 1957).

[208h] Variations de couple dues aux variations des pressions - Méthode de calcul et application $\grave{a}$ un groupe bulbe de $N=20000 \mathrm{~kW}$. R 4656 (octobre 1957).

[208i] . Note sur le calcul des pulsations de puissance d'une turbine soumise à l'effet de la houle. R 4716 (janvier 1958).

[208j] Comportement à la houle du caisson-usine, en l'absence du débit de la turbine. R 4717 (décembre 1957).

[208k] Détermination approchée du spectre d'énergie. R 7357 (février 1960)

[208l] Essai de reproduction des houles de la région de Chausey. R 7457 (avril 1960).

[209] Corne (A.) et Bel.tier (J.). - Note sur les études de l'aménagement (1942-1955) (août 1955).

[210] Note sur la conception de l'usine marémotrice Chausey - Mont-Saint-Michel, S.E.U.M., LV/DP (8-11-1955).

[211] Note succincte sur les principaux renseignements recueillis par S.E.U.M. sur le régime de la houle en baie de Chausey - Mont-Saint-Michel. S.E.U.M., note n"1, GWD/DP (27-11-1955); note $n^{\prime \prime} 2$, GWP/DP (12-12-1955).

[212] Debyser (J.). - Etudes sur les sédiments actuels recueillis dans les forages exécutés entre les îles Chausey et Granville. Rapport de I'Institut Français du Pétrole, n" 774 (janvier 1956).

[213] Les projets établis par les Entreprises S.G.E.-Fougerolle :

[213a] Premier projet avec groupes bulbes amont de $7000 \mathrm{~kW}$ du type Rance (1955)

[213b] Avant-projet 1955-56 (juin 1956)

[213c] Solution d'une usine souterraine entre Grouin et Chausey (janvier 1957)

[213d] Exécution par caisson. Première étude préliminaire (20 février 1957).

[213e] Exécution par caisson. Première étude préliminaire (octobre 1957). 
[214] Aménagement de l'usine entre des batardeaux. Grands Travaux de Marseille (15 mai 1957) et dossier com-

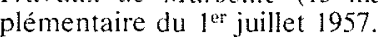

[215] Aménagement de Chausey. E.D.F., Région d'Equipement Hydraulique, avant-projet $n^{\circ} 8$, Dinard (mars 1958).

[216] Rapports du Laboratoire National d'Hydraulique sur le modèle réduit de la Manche :

[216a] $1^{\text {er }}$ compte rendu d'essais, B 527 (17 janvier 1956).

[216b] $2^{e}$ compte rendu d'essais, B 527 (19 juin 1956).

[216c] $3^{\mathrm{e}}$ compte rendu d'essais, T 90 (1 $1^{\mathrm{er}}$ avril 1957).

$[216 d] 4^{e}$ compte rendu d'essais, T 117 ( $^{\text {er }}$ novembre 1957).

[217] Rapports du Laboratoire National d'Hydraulique sur le modèle réduit des îles Chausey :

[217a] Rapport $n^{\circ} 1$. - Construction du modèle (1 juillet 1959). [217b] Rapport $\mathrm{n}^{\circ} 2$. - Les conditions naturelles du golfe de Saint-Malo: les mouvements du plan d'eau. T. 163 (1 ${ }^{\text {er }}$ octobre 1960).

[217c] Rapport $n^{\prime \prime} 3$. - Les conditions naturelles du golfe de Saint-Malo: les courants de marée. T 169 (1 ${ }^{\mathrm{er}}$ août 1961).

[217d] Rapport $n^{\prime \prime} 4$. Etude énergétique de la marée dans le golfe de Saint-Malo. T 315 (20 décembre 1963).

[218] Sondages dans la baie du Mont-Saint-Michel, Le Ludion, FV/SD ( 9 octobre 1961).

[219] Bonnefille (R.) et JEAnNel (M.). - Etude de l'aménagement d'un port de plaisance dans l'anse de Herel. Laboratoire National d'Hydraulique, T DHM 531 (avril 1967).

[220] Etude de l'usine marémotrice des îles Chausey sur modèle tournant de la Manche. Laboratoire National d'Hydraulique. T $603, \mathrm{HC} 031$ (juin 1968).

\section{Planches}

Etude statistique de la houle :

— au sud de Chausey ........................ 1-2

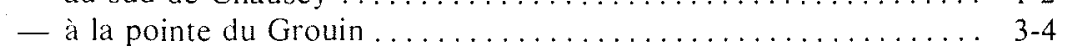

- à Granville .............................. 5-6

Plans de vagues :

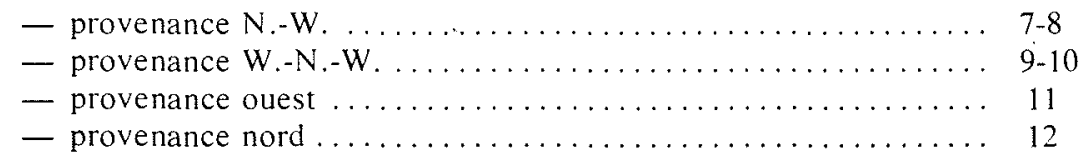




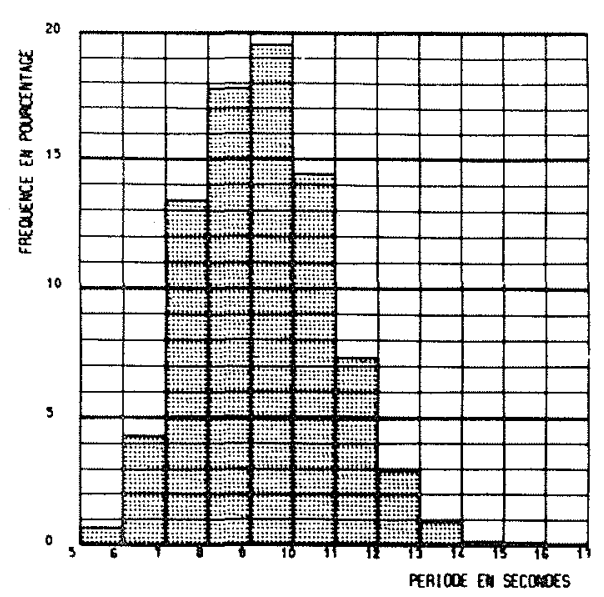

histogramme de la periode movenne

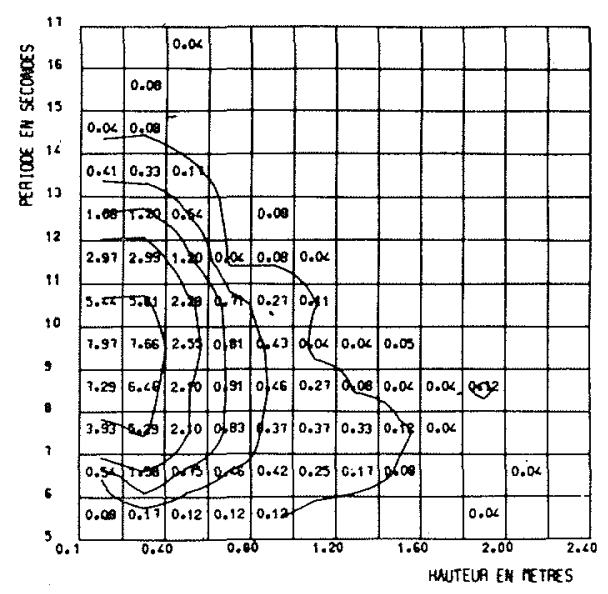

CORRELATION HAUTEUR-PERIOOE MOYENNES

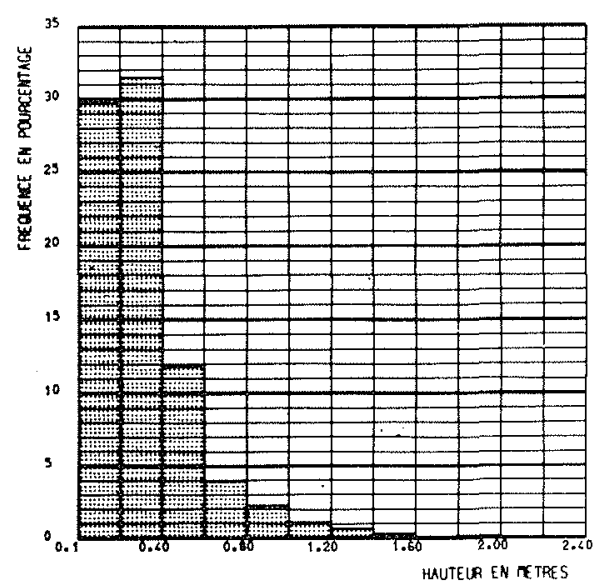

0 0-0 A3- A- E DE LA HAUTEUR MOYEnNe

\begin{tabular}{|c|c|c|}
\hline \multicolumn{2}{|c|}{ MOUILLAGE } & PERIDOE ANALYSEE \\
\hline 1 & 10 & $27-6-56$ А $25-8-56$ \\
\hline 2 & 10 & $28-8-56 \mathrm{AU} 12-10-56$ \\
\hline 3 & 10 & $13-10-56$ a $3-12-56$ \\
\hline 6 & 10 & $18-12-56$ N $19-1-57$ \\
\hline 5 & 10 & $7-2-57$ \&U $20-5-57$ \\
\hline 6 & 10 & $24-5-57$ \&u $23 \cdot 7-53$ \\
\hline 7 & 10 & $23=7-53$ a $5-5-10-57$ \\
\hline 8 & 10 & $8-10-57$ AU $2-12-57$ \\
\hline 9 & 10 & $4-12-57 \quad A \cup 23-1-58$ \\
\hline 10 & 10 & $29-1-58$ AU 17-3-58 \\
\hline 11 & 10 & $20-3-58 \mathrm{~A} \cup 1-7-58$ \\
\hline 12 & 10 & 5- $7-59 \AA \cup 25-8-58$ \\
\hline 13 & 10 & $29-8-59 \mathrm{A \cup} 2<-10-58$ \\
\hline 14 & 10 & $\angle-11-58 \wedge \cup 5-1-59$ \\
\hline 15 & 10 & $1<-1-59 \wedge \cup \div 9-2-59$ \\
\hline 16 & 10 & 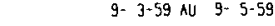 \\
\hline 17 & 10 & $15-5-59 \mathrm{~A} \cup<-9-59$ \\
\hline 18 & 10 & $12-10-59 \mathrm{k} \cup 23-11-59$ \\
\hline 19 & 10 & $19-12-59 \propto \cup<8-1-60$ \\
\hline 20 & 10 & $24-1-60 \mathrm{~N} \cup 29-2-60$ \\
\hline 21 & 10 & $5-3-60$ AU $<-<-60$ \\
\hline
\end{tabular}

ENREG ISTREMENT DE LA HOULE A SUD DE CHAUSEY

$$
\text { DU 27-6-56 AU 4- 4-60 }
$$

1204 JOURS D OBSERVATION - 223 JOURS DE CALME PLAT $(18.51)$

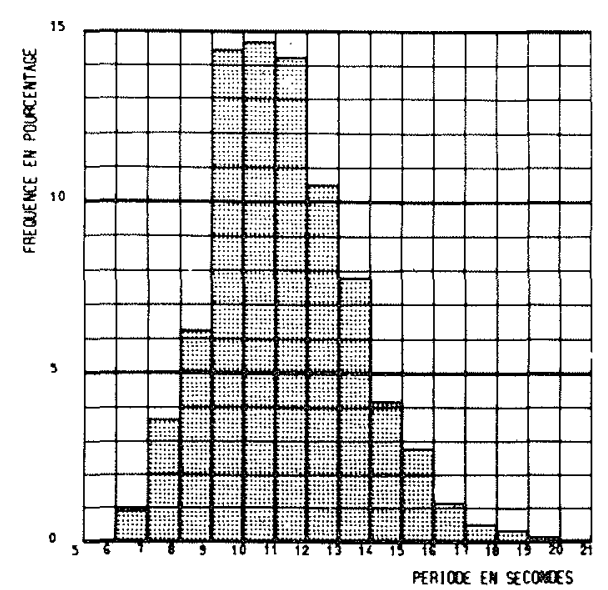

HISTOGRAMME OE LA PERIODE MAXIMALE

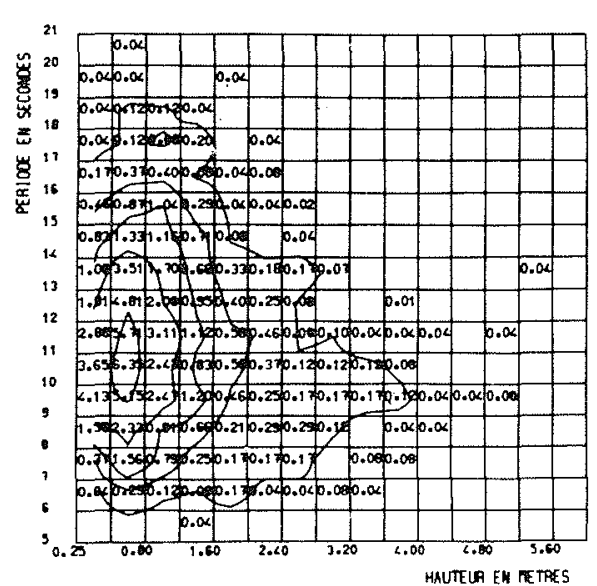

CORRELATION HAUTEUR-PERIODE MAXIMALES

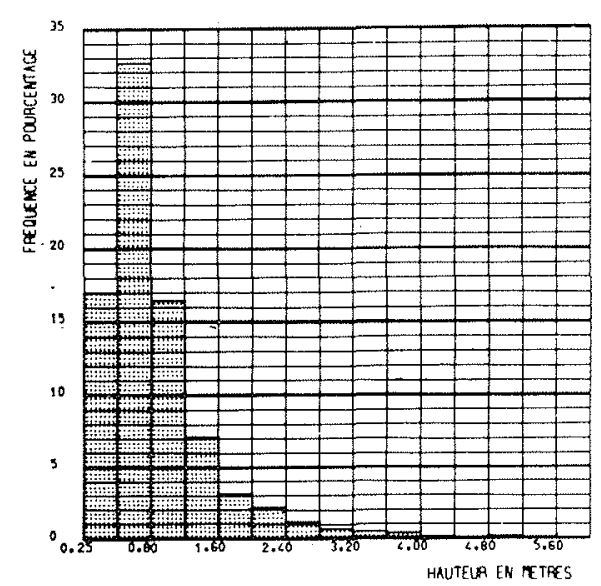

HISTOGRAMME OE LA HAUTEUR MAXIMALE

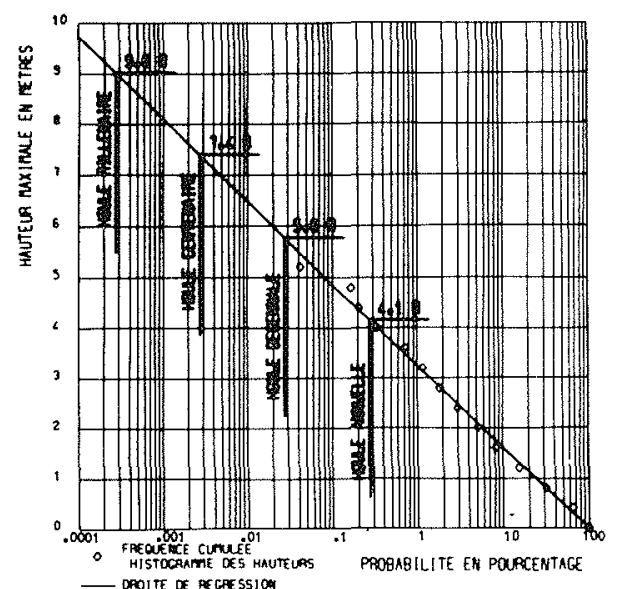

EXTRAPOLATION A LA HOULE CENTENAIRE ET MILLENAIRE
ENREG ISTREMENT DE LA HOULE A SUD DE CHAUSEY

$$
\text { DU 27-6-56 AU 4-4-60 }
$$

1204 JOURS D OBSERVATION - 223 JOURS DE CALME PLAT $118.5,1$ 


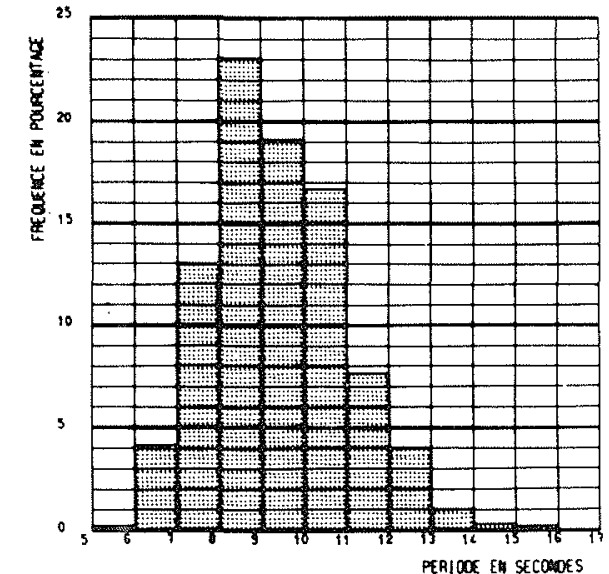

HISTOGRAMME DE LA PERIODE MOYENNE

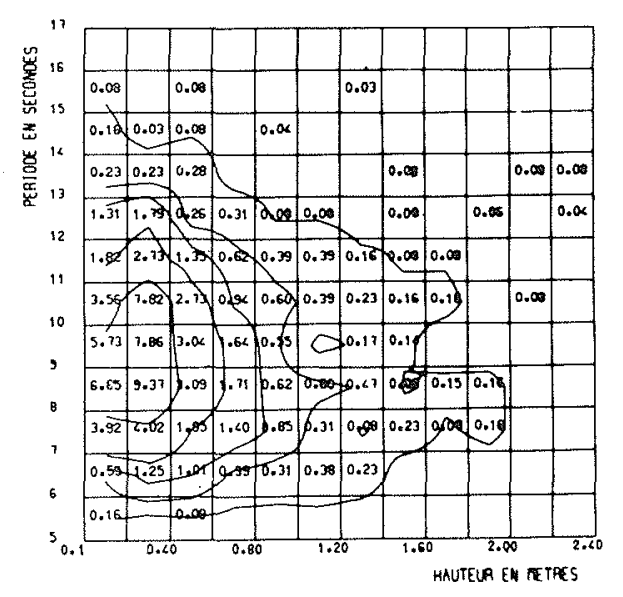

CORRELATION HAUTEUR-PERIDOE MOYENNES

ENREGISTREMENT DE LA HOULE A POINTE DU GROIN

$$
\text { DU } 6-2-57 \text { AU } 4-4-60
$$

642 JOURS D OBSERVATION - 69 JOURS DE CALME PLAT $(10.7$,
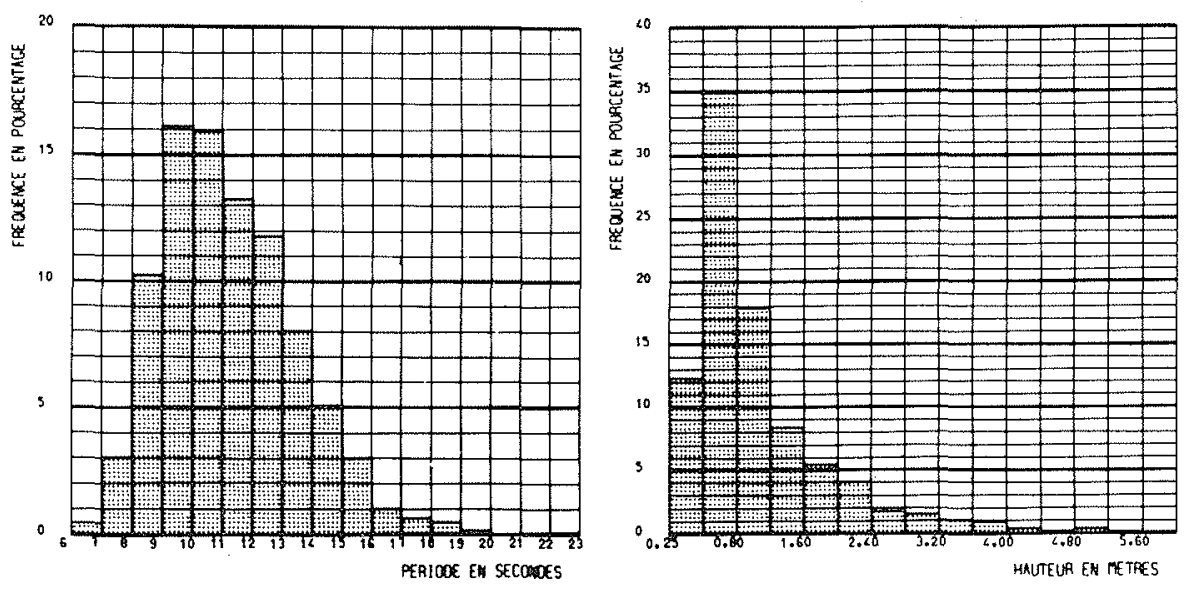

hISTOGRAMME DE LA PERIODE MAXITALE

HISTOGRAMTE DE LA HAUTEUR MAXIMALE

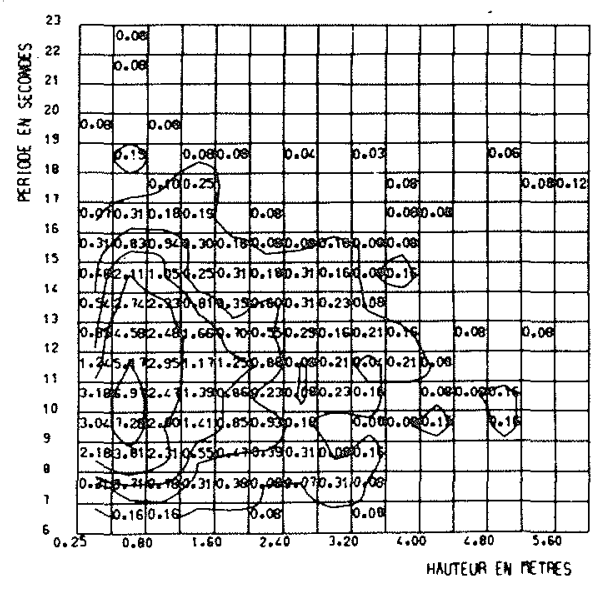

CORRELATION HAUTEUR-PERIOOE MAXIMALES

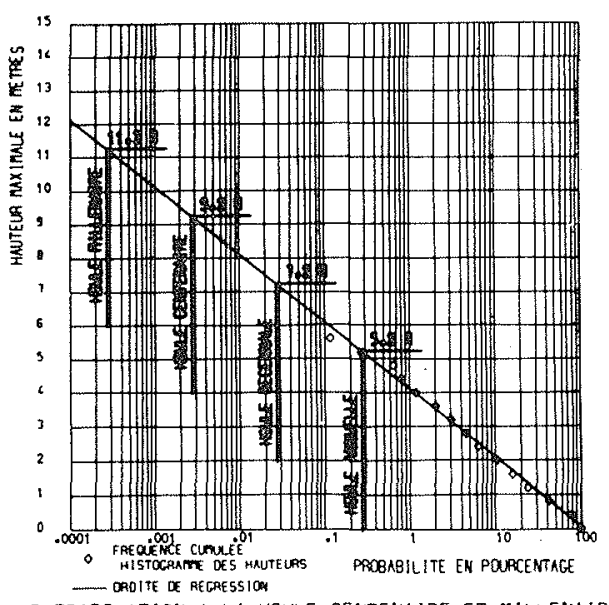

ENREgISTREMENT DE LA HOULE A POINTE OU GROIN DU $6-2-57$ AU $4-4-60$

642 JOURS D OBSERVATION - 69 JOURS DE CALME PLAT 110.71$)$ 


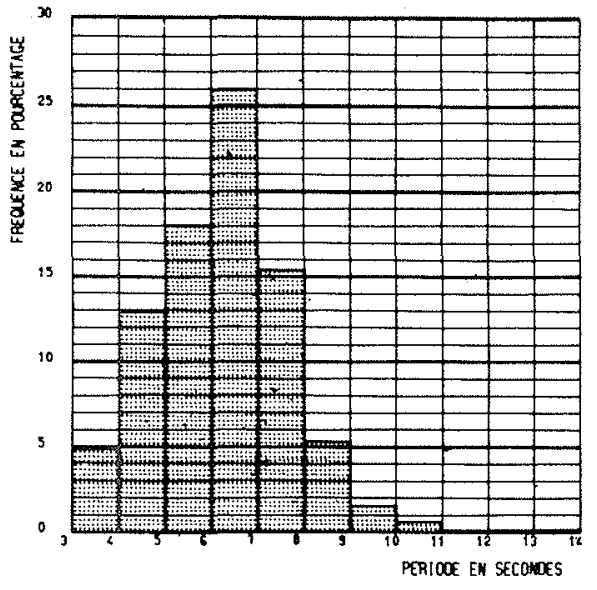

histogramme de LA PERIODE MOYENNE

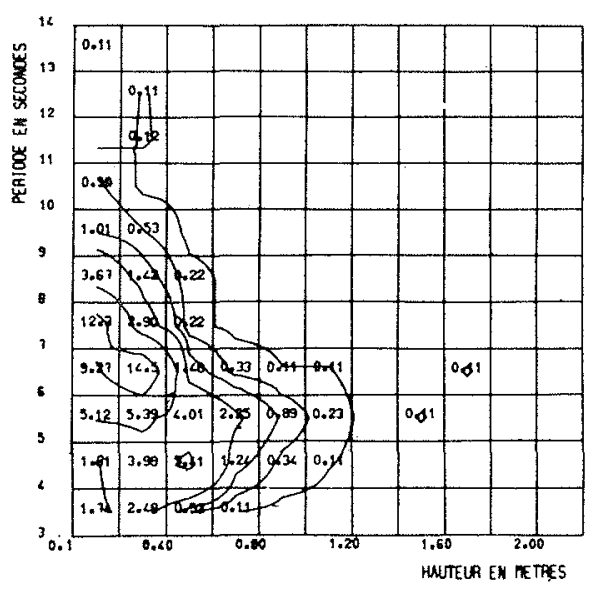

CORRELATION HAUTEUR-PERIODE MOYENNES

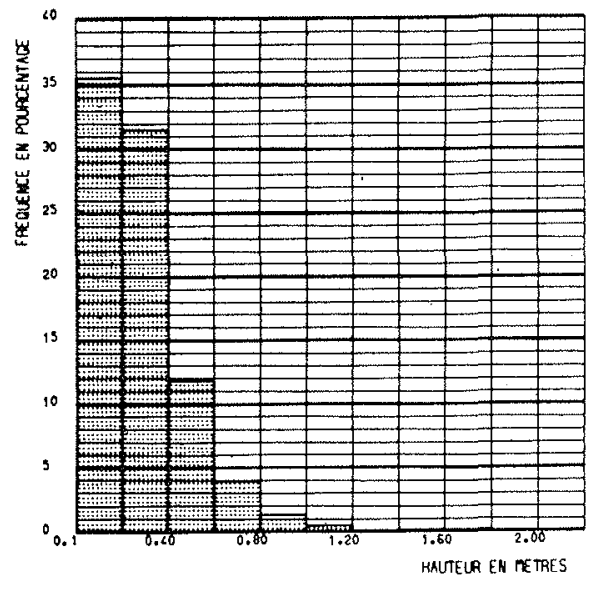

O $0-0$ 13- A- E DE LA HAUTEUR MOYENNE

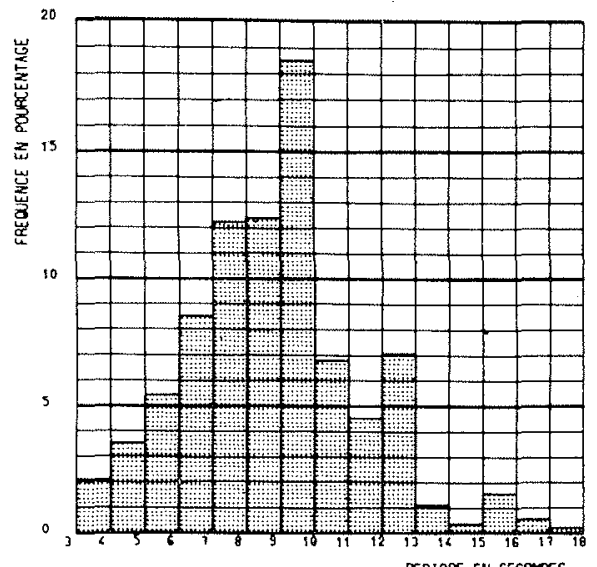

PerIOOE EN SECOAOCS

HISTOGRAMME OE LA PERIODE MAXIMALE

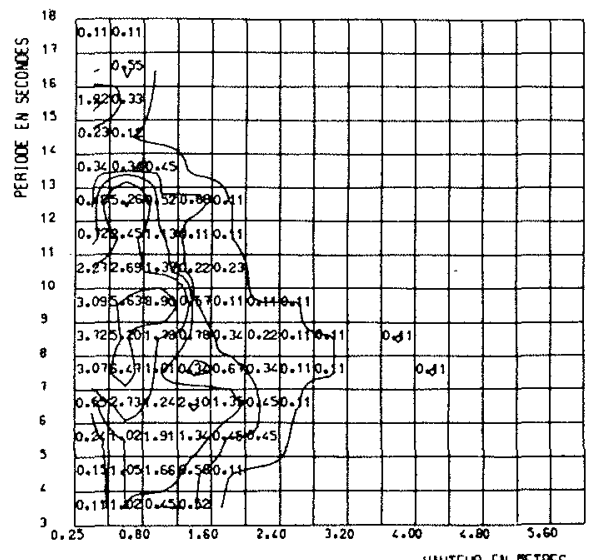

CORRELATION HAUTEUR-PERIODE MAXIMALES

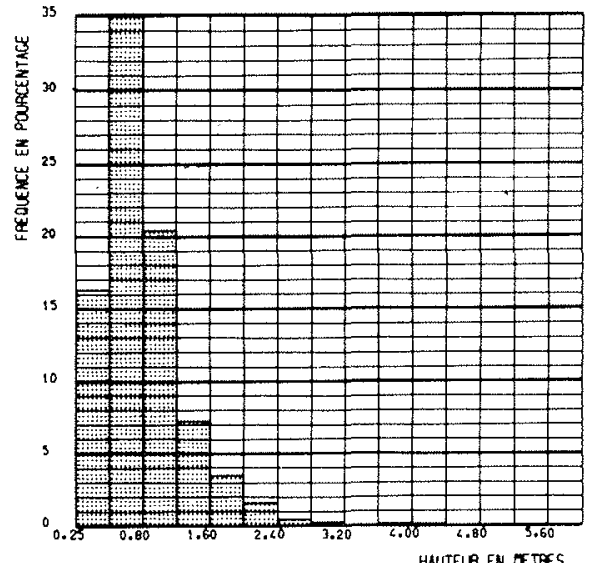

HRUTER EN FTTES

HISTOGRAMME DE LA HAUTEUR MAXIMALE

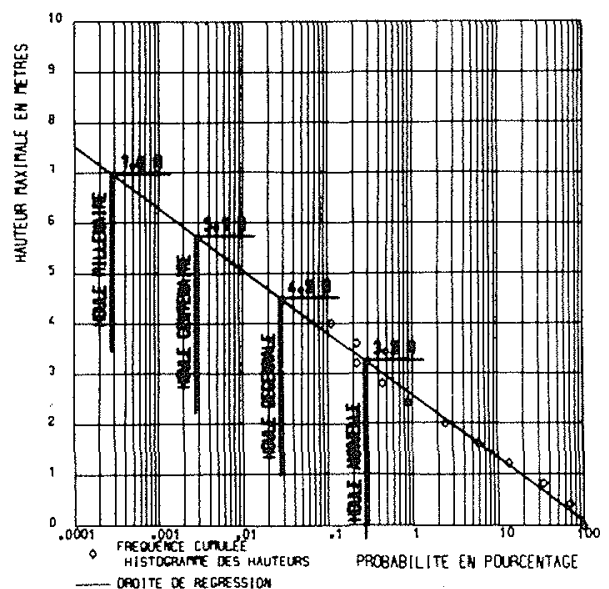

EXTRAPOLATION A LA HOULE CENTENAJRE ET MILLENAIRE
ENREg ISTREMENT DE LA HOULE a gRANVILLE

$$
\text { DU 30-7-65 AU 10-8-66 }
$$

229 JOURS D OBSERVATION - 35 JOURS DE CALME PLAT $(15.11)$
EnREg Istrement de la hOULE a granville

$$
\text { DU 30-7-65 AU } 10-8-66
$$

229 JOURS D OBSERVATION - 35 JOURS DE CALME PLAT $(15.11$ 


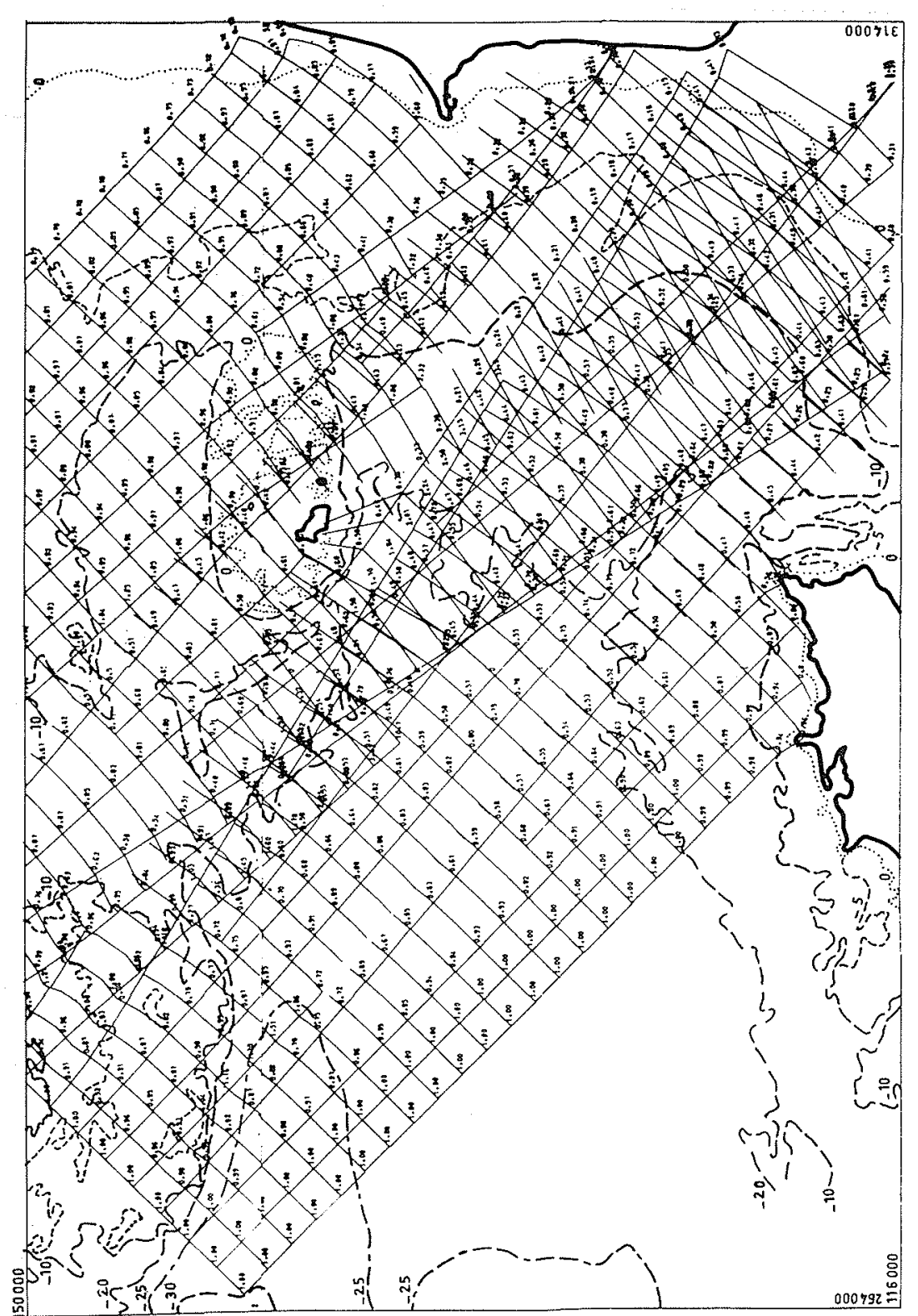

HOULE DE PROVENANCE NORD-OUEST-PERIODE $6 \mathrm{~s}$. NIVEAU DE LA MER $+12,6 \mathrm{~m}$. CM

PL.7

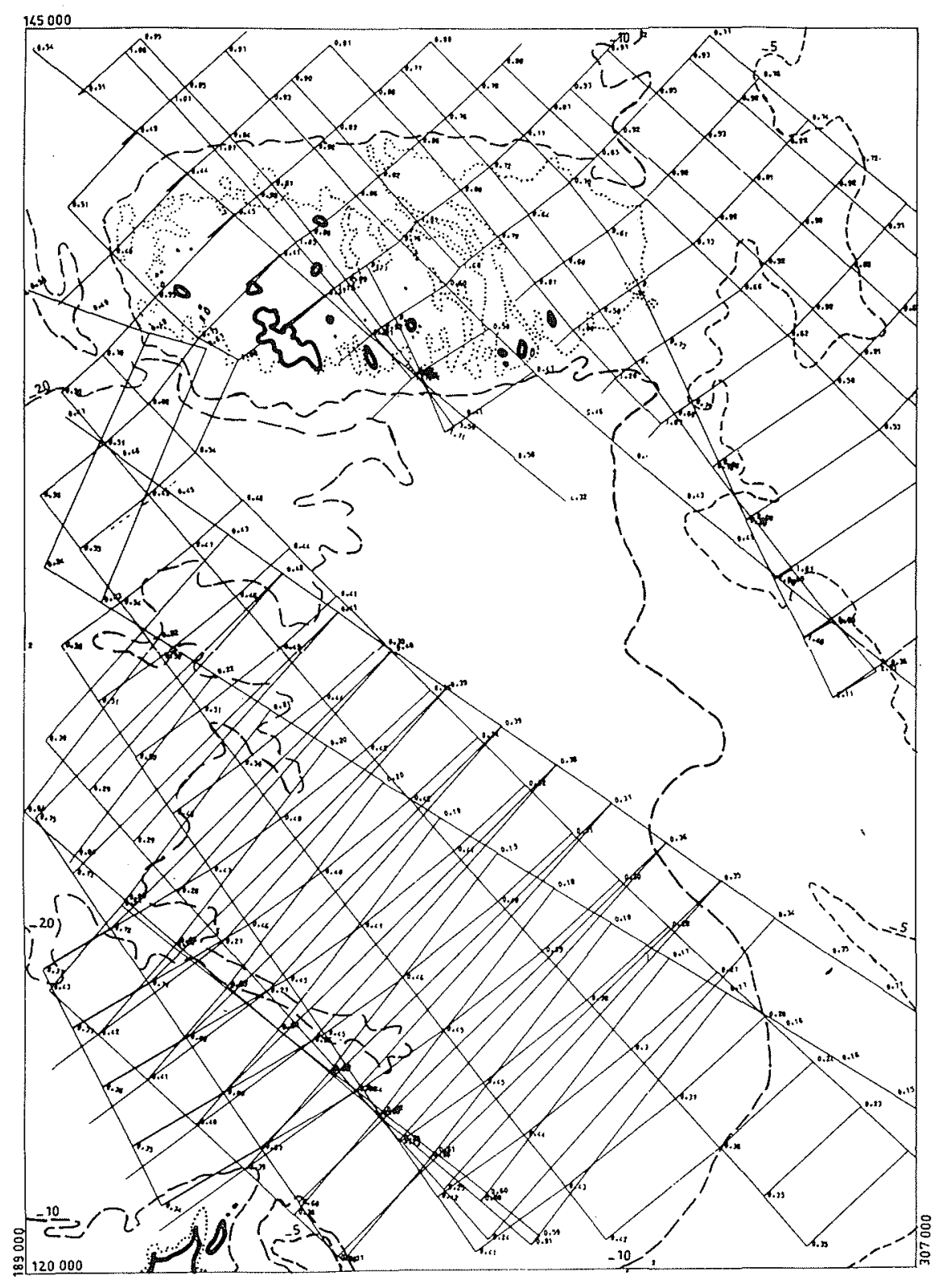

HOULE DE PROVENANCE NORD-OUEST - PERIODE 65. NIVEAU DE LA MER $+12,6 \mathrm{~m}$. CM

Echelle : 1/160 000

Cotes C.M. 


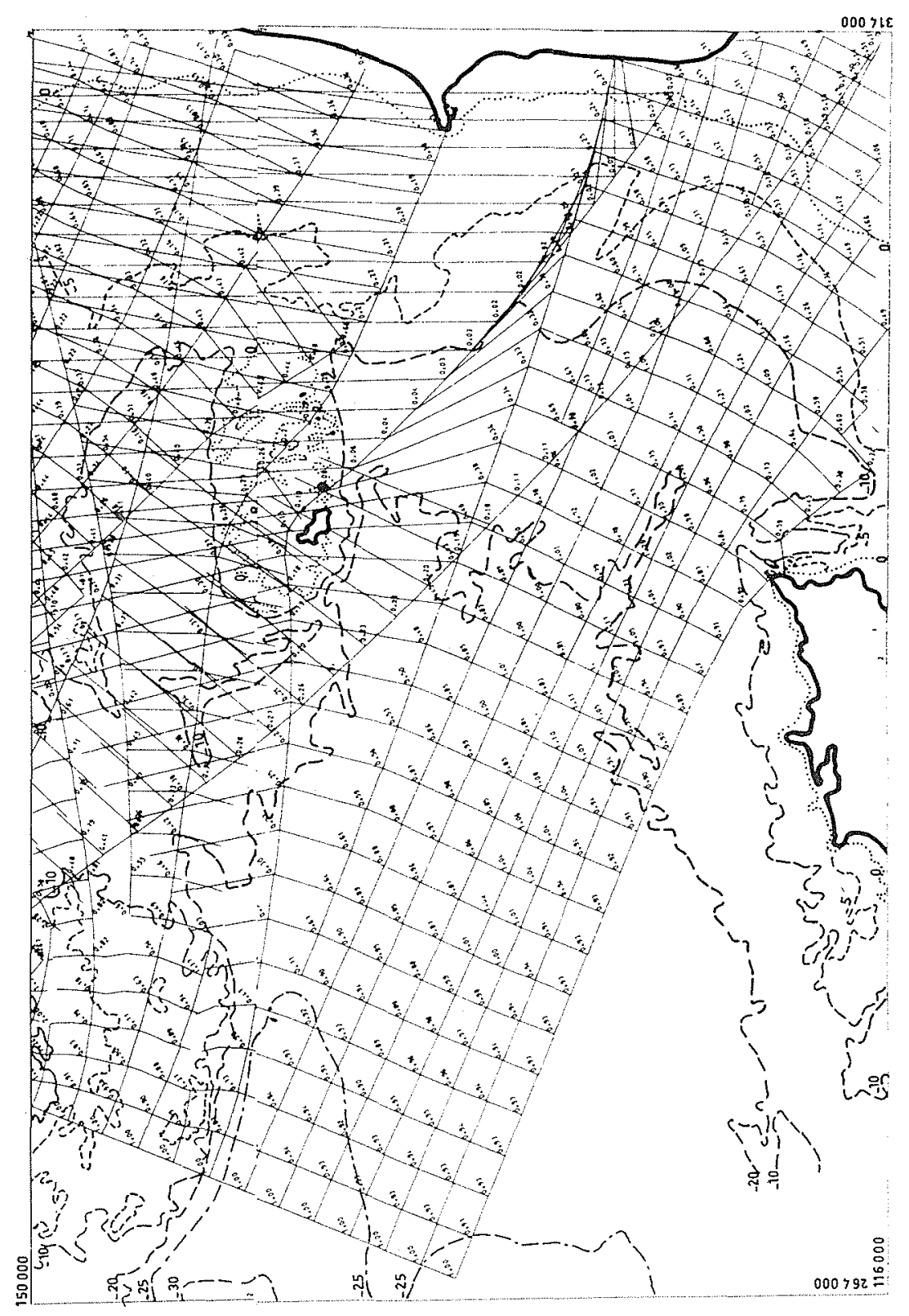

HOULE DE PROVENANCE OUEST-NORD-OUEST-PERIODE $10 \mathrm{~s}$. NIVEAU DE LA MER $+13.6 \mathrm{~m}$. CM

Pl.9
Echelle : 1/320 000 Cotes C.M.

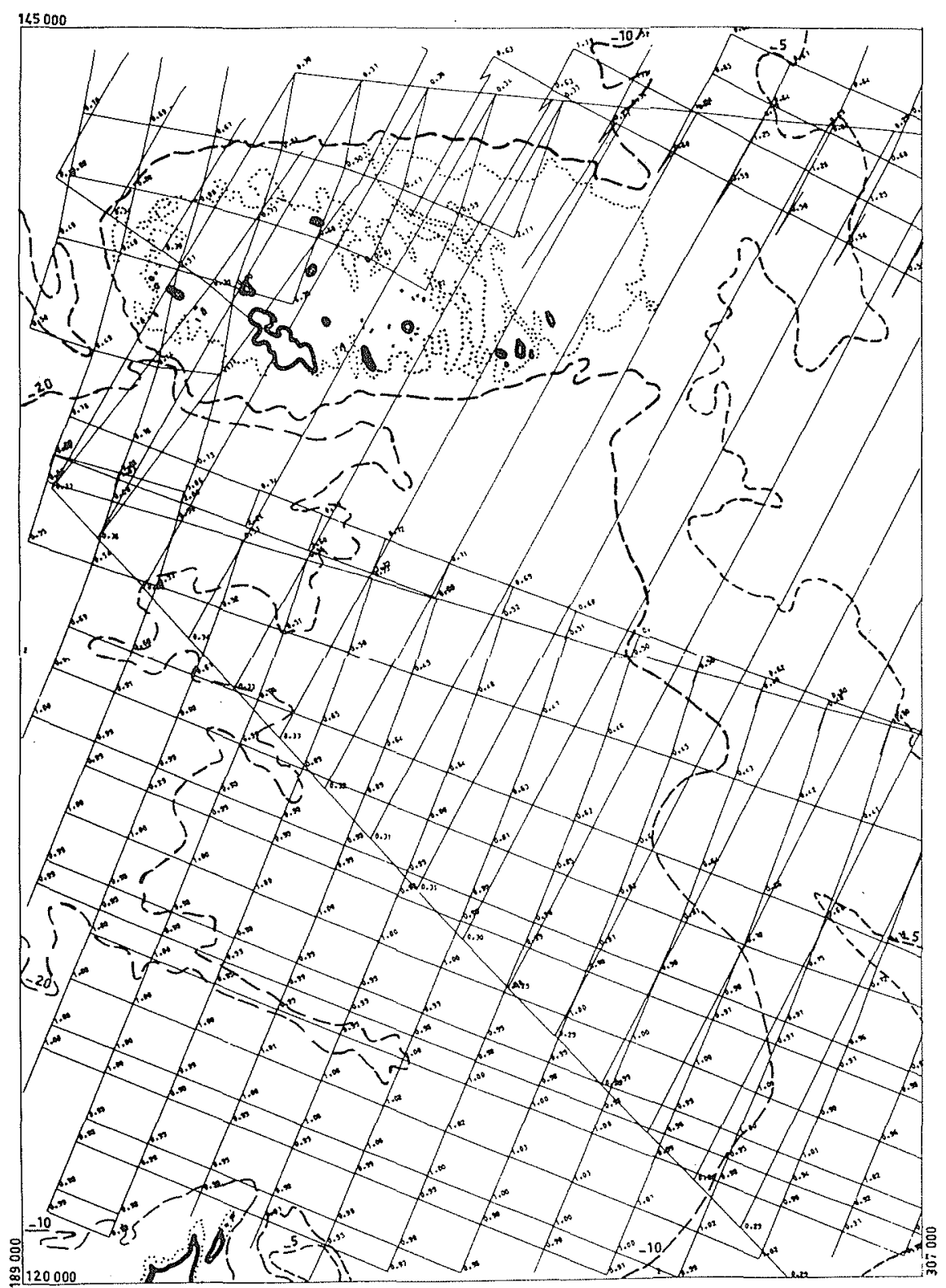

HOULE DE PROVENANCE OUEST-NORD-OUEST-PERIODE 65. NIVEAU DE LA. MER $+12.6 \mathrm{~m}$. CM.

Echelle : 1/160 000 


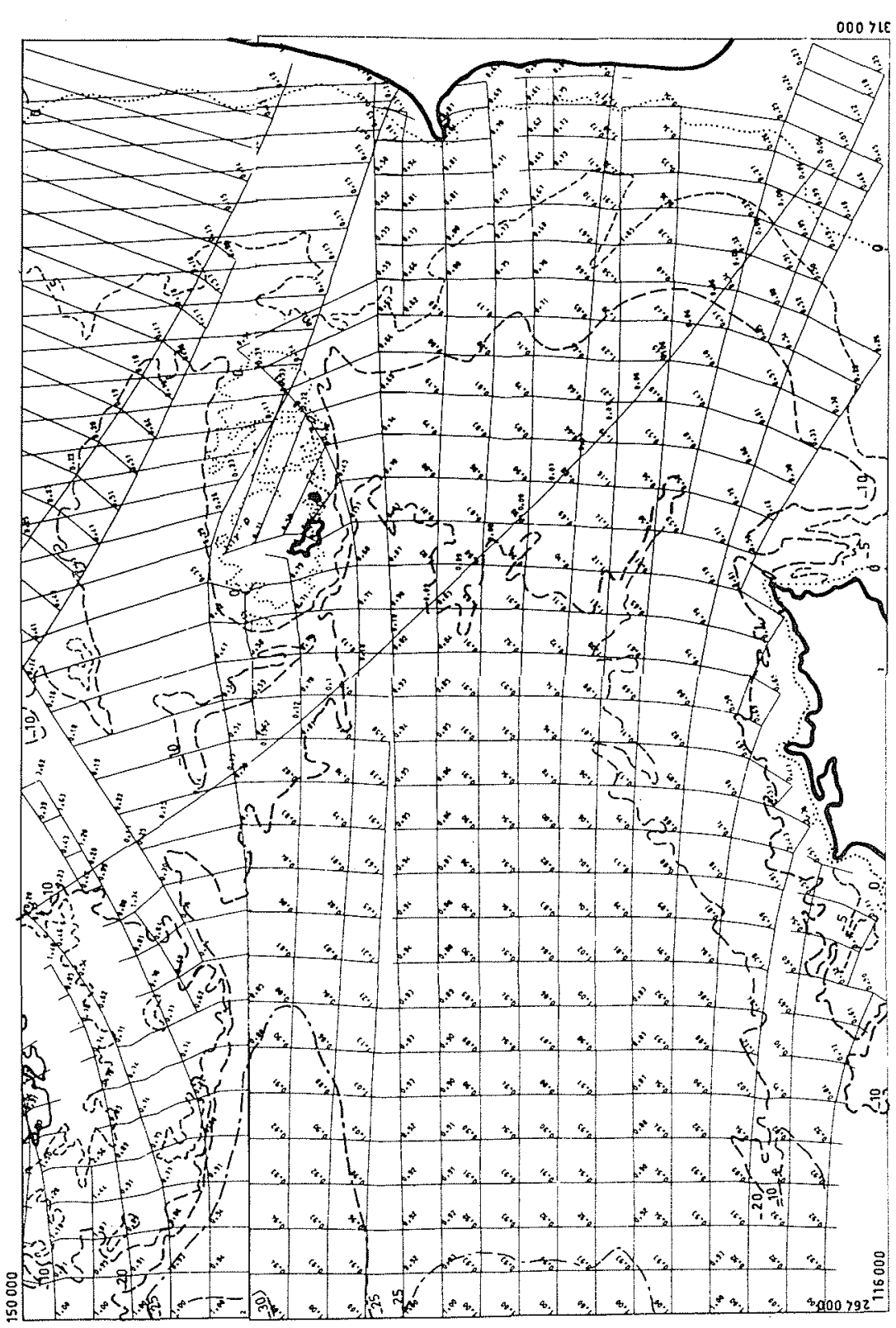

HOULE DE PROVENANCE OUEST - PERIODE $10 \mathrm{~s}$. NIVEAU DE LA MER + $13.6 \mathrm{~m}$. CM.

PL. 11

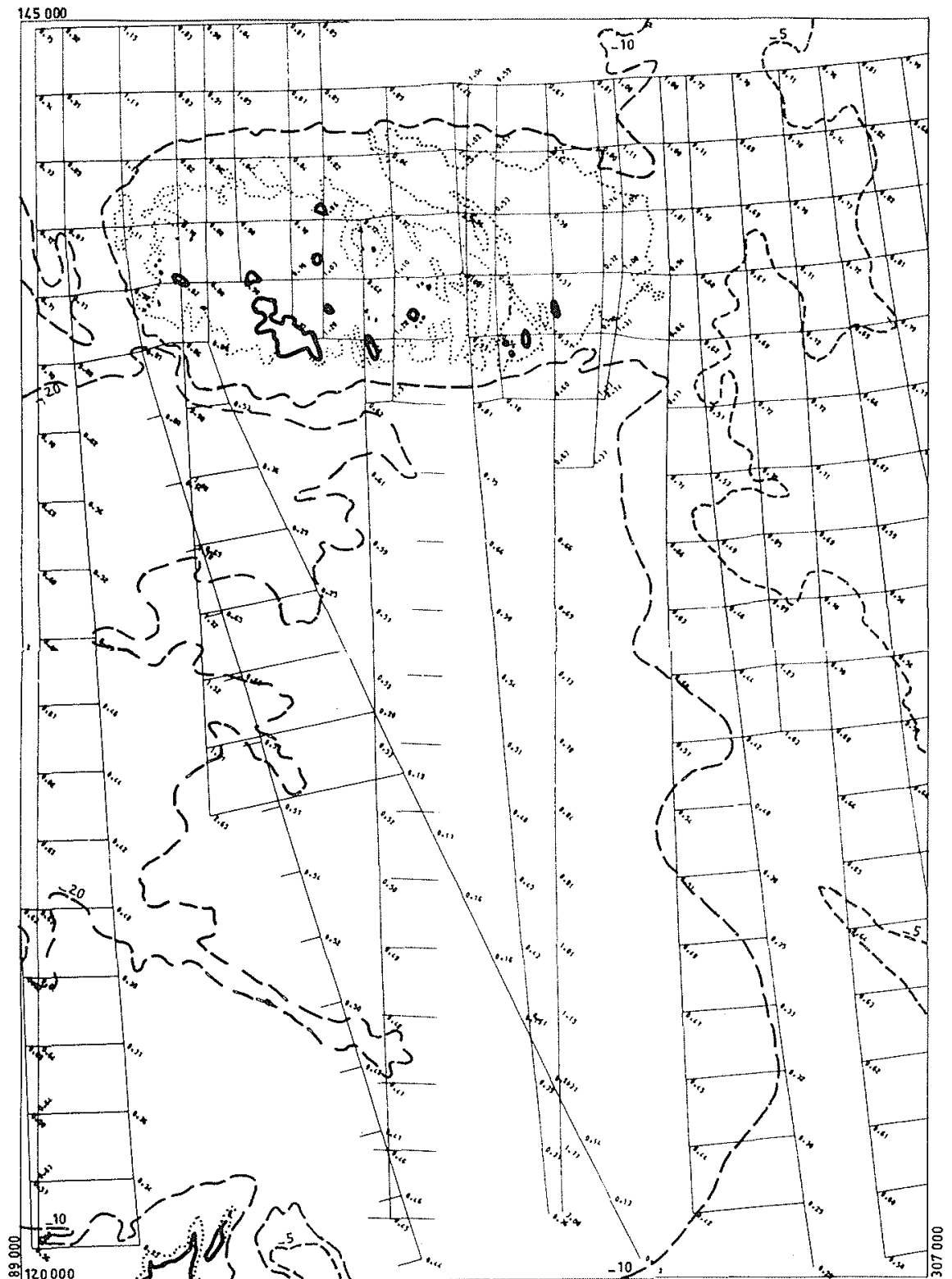

HOULE DE PROVENANCE NORD - PERIODE $6 \mathrm{~s}$. NIVEAU DE LA MER $+12.6 \mathrm{~m}$. CM.

Echelle : 1/160 000 cotes C.M. 